\title{
Availability Analysis for the Quasi-Renewal Process
}

\author{
Ian Jon Rehmert \\ Dissertation submitted to the Faculty of the \\ Virginia Polytechnic Institute and State University \\ in partial fulfillment of the requirements for the degree of
}

\section{Doctor of Philosophy \\ in}

Industrial and Systems Engineering

\author{
Joel A. Nachlas, Chair \\ C. Richard Cassady \\ C. Patrick Koelling \\ Keying Ye \\ Ebru Bish
}

October 9, 2000

Blacksburg, Virginia

Keywords: reliability, corrective maintenance, preventive maintenance, availability, imperfect repair, general repair, minimal repair, quasi-renewal process, non-homogeneous process 


\title{
Availability Analysis for the Quasi-Renewal Process
}

\author{
Ian Jon Rehmert
}

\section{(ABSTRACT)}

The behavior of repairable equipment is often modeled under assumptions such as perfect repair, minimal repair, or negligible repair. However the majority of equipment behavior does not fall into any of these categories. Rather, repair actions do take time and the condition of equipment following repair is not strictly "as good as new" or "as bad as it was" prior to repair. Non-homogeneous processes that reflect this type of behavior are not studied nearly as much as the minimal repair case, but they far more realistic in many situations. For this reason, the quasi-renewal process provides an appealing alternative to many existing models for describing a non-homogeneous process. A quasi-renewal process is characterized by a parameter that indicates process deterioration or improvement by falling in the interval $[0,1)$ or $(1, \infty)$ respectively. This parameter is the amount by which subsequent operation or repair intervals are scaled in terms of the immediately previous operation or repair interval. Two equivalent expressions for the point availability of a system with operation intervals and repair intervals that deteriorate according to a quasi-renewal process are constructed. In addition to general expressions for the point availability, several theoretical distributions on the operation and repair intervals are considered and specific forms of the quasirenewal and point availability functions are developed. The two point availability expressions are used to provide upper and lower bounds on the approximated point availability. Numerical results and general behavior of the point availability and quasirenewal functions are examined. The framework provided here allows for the description and prediction of the time-dependent behavior of a non-homogeneous process without the assumption of limiting behavior, a specific cost structure, or minimal repair. 


\section{Acknowledgements}

I would first like to thank my advisor, Joel Nachlas, for his guidance, support, and friendship throughout my graduate and undergraduate studies over the past nine years. His influences in the classroom, as an advisor, and as a coach on the lacrosse field have had an extremely significant and positive impact on my life. I would also like to thank my committee members, Richard Cassady, Pat Koelling, Keying Ye, Ebru Bish, and John Kobza for their contributions and for their flexibility and understanding in helping me meet such an ambitious schedule.

The foundation for my education and success started at home. I credit my parents, John Rehmert and Linda Middlestadt, and Ellen Baese for shaping me into the person I am today. Their unwavering love and support throughout my life has given me the confidence and ability to pursue my academic and personal interests.

I would like to give a special thanks to Richard Cassady for taking an interest in my academic development, for his friendship, and for making the trip from Arkansas and Mississippi to serve on my committee. I thank him for our several conversations over the years that provided me with much needed reassurance and helped me put things in perspective. Through their encouragement and actions as role models and mentors, Joel and Rich are both responsible for fostering my interest and success in the graduate program at Virginia Tech.

The faculty and staff of the Industrial and Systems Engineering (ISE) department have made my experience at Virginia Tech rewarding and enjoyable. I would like to thank John Casali and the faculty of the ISE department for providing financial support and teaching opportunities throughout my time as a graduate student. I would like to give a special thanks to Lovedia Cole, Latanya Walker, and Sonya Dye. They always pointed me in the right direction and provided me with a patient, understanding, and sympathetic ear on countless occasions. In addition, the numerical work contained in this dissertation 
would not have been possible without the help of Pat Koelling, Will Vest, and Brian Burgess and the resources in the ISE Simulation and Optimization Laboratory.

During my graduate study, I held a position with the Institute for Defense Analyses (IDA). I thank Robert Roberts, Thomas Christie, and Ed Smith for providing me with such an outstanding and rewarding opportunity. In particular, Edward Smith, Alton Wallace, Lee Carr, and Richard White took a special personal interest in my professional development and provided me with advice and friendship that I will always value. In addition, many others enhanced my experience at IDA. I include in this list: Bernard Kempinski, Arthur Fries, Lucy Harris, Donnie McKethan, Brenda Howard, and Joel Christenson.

Throughout the past several years, three very special people in my life have provided me with invaluable friendship, positive reinforcement, and unconditional assistance with any and all aspects of my life. These three people are Marcel Messing, Anne Tiburzi, and my brother John Rehmert. I would also like to thank James and Rosalind Messing for their warmth and hospitality on several occasions.

Of my many friends and colleagues, Dave Franz and Lisa Maillart are friends that I have been fortunate enough to enjoy both my academic and personal life with. Their unique ability to understand me has been invaluable over the years.

One important aspect of enjoying success is to have people that you can share it with. All of the people mentioned above fall into this category, but I would also like to mention several others that are very important to me as well. They are friends, family, roommates, lacrosse teammates, Delta Upsilon fraternity brothers, and colleagues. In this group I include: Susan Middlestadt, Ursula Middlestadt, Jeff Edwards, Jennifer Edwards, Marv Edwards, Brad Edwards, Dave and Stacey Ferris, Craig and Kim Elliott, Matt Garcia, Christine Kennedy, Justin and Christine Ellenberger, Ken Tortolani, Bill Fennell, Dave Howard, Matt Swartz, Mike and Cindi Hane, Pat and Yvette Mulholland, Jeff Muldoon, Darah Hartman, Adam Randall, Paul Thompson, Brian Wilson, Andy 
Woerner, Chris Carlton, Cole Smith, Barb Fraticelli, Elise Caruso, Mardi Russell, Greg Beskow, Darrall Henderson, Jared and Kalie Stokes, David Curnow, Rachael Schroeder, Adrienne Dedea, Kim Arnold, Carter Shields, Carlos Torres, John Villecco, Jon Beckwith, Ryan Walker, Mike Stewart, Justin Herzog, Craig Morin, Darren Drewry, Jeff Leidy, Jacqueline Doucette, Cindy Moran, Sean Davies, Scott Mealey, George Ioannou, Chris Stewart, Jay Collins, Josette Oder, Sarah Stollhans, Colleen Ryan, Adam Lipin, Jeremy Guerin, Brad Jones, Mike Keyes, Margaret Keyes, Brad Spain, and all the rest of the lacrosse family, Clay Street Crew, and Delta Upsilon Fraternity at Virginia Tech.

I thank all of you again and hope that some day I can return the favor! 


\section{Table of Contents}

Chapter 1 - Introduction ............................................................................. 1

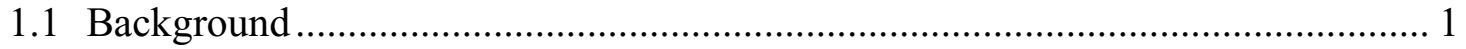

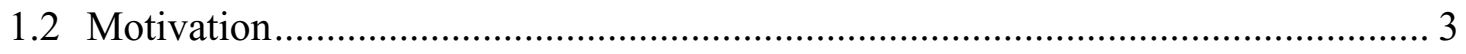

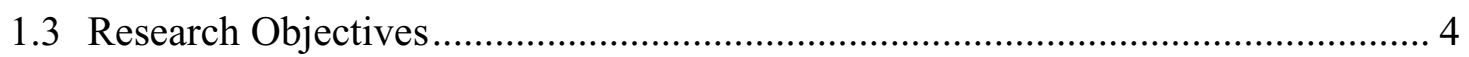

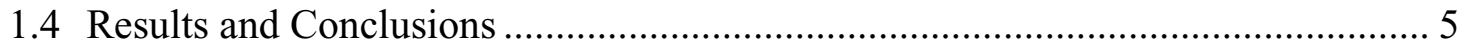

Chapter 2 - Literature Review................................................................... 7

2.1 Introduction ......................................................................................................... 7

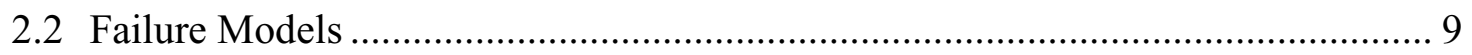

2.3 Preventive Maintenance................................................................................... 12

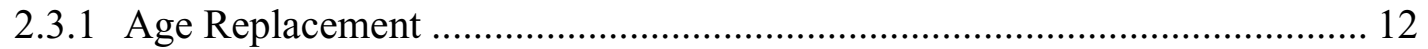

2.3.2 Block Replacement............................................................................... 13

2.3.3 Imperfect Preventive Maintenance ............................................................. 14

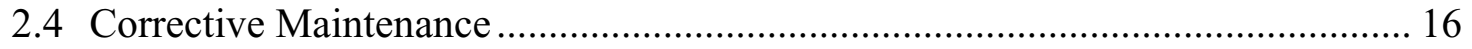

2.4.1 Perfect Repair ................................................................................. 17

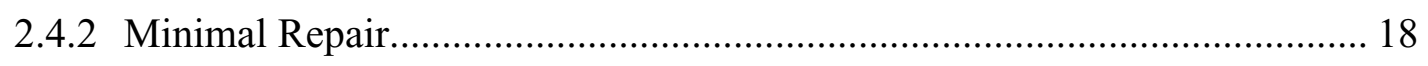

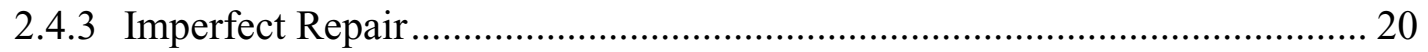

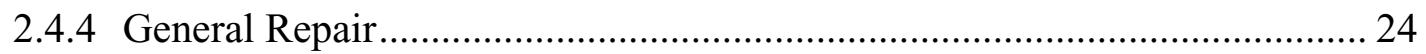

2.4.4.1 Virtual Age Model ....................................................................... 24

2.4.4.2 Scale Factor Model ......................................................................... 27

2.4.4.3 Additive Age Model ..................................................................... 27

2.4.4.4 $(\alpha, \beta)$ Model............................................................................... 28

2.4.4.5 Improvement Factor Model ......................................................... 29

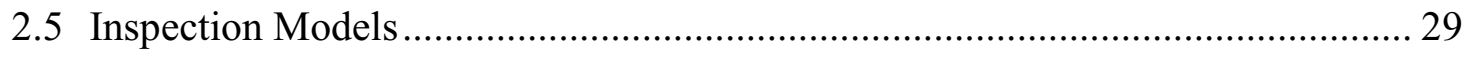

Chapter 3 - Methodology....................................................................................... 32

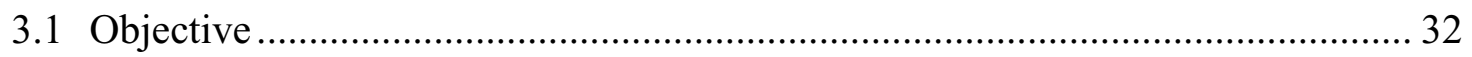

3.2 Quasi-renewal Process .................................................................................... 33

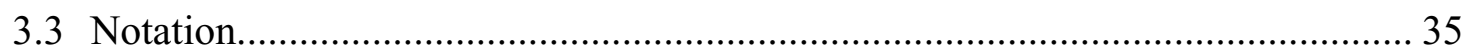

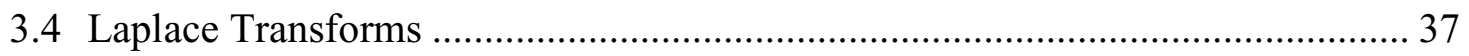




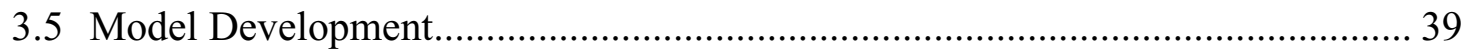

3.5.1 The Quasi-renewal Function, $Q(t)$........................................................ 39

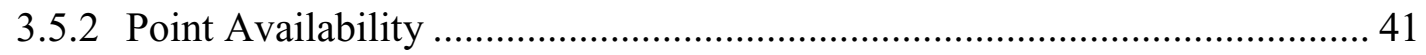

3.5.3 Bounds on the Point Availability ................................................................ 44

3.6 Quasi-Renewal Model Under Exponential Operating Intervals ........................... 46

3.6.1 Exponential Quasi-Renewal Function....................................................... 47

3.6.2 Uptime-Based Point Availability Function with Exponential Repair ........... 47

3.6.3 Downtime-Based Point Availability Function with Exponential Repair ...... 48

3.7 Quasi-Renewal Model Under Normal Operating Intervals ................................... 49

3.7.1 Normal Quasi-Renewal Function ............................................................ 50

3.7.2 Uptime-Based Point Availability Function with Normal Repair ................... 50

3.7.3 Downtime-Based Point Availability Function with Normal Repair ............. 53

3.7.4 Uptime-Based Point Availability Function with Exponential Repair ........... 54

3.7.5 Downtime-Based Point Availability Function with Exponential Repair ...... 56

3.8 Quasi-Renewal Model Under Gamma Operating Intervals .................................. 56

3.8.1 Gamma Quasi-Renewal Function ............................................................ 57

3.8.2 Uptime-Based Point Availability Function with Gamma Repair.................. 57

3.8.3 Downtime-Based Point Availability Function with Gamma Repair............. 58

3.8.4 Uptime-Based Point Availability Function with Exponential Repair ........... 59

3.8.5 Downtime-Based Point Availability Function with Exponential Repair ...... 60

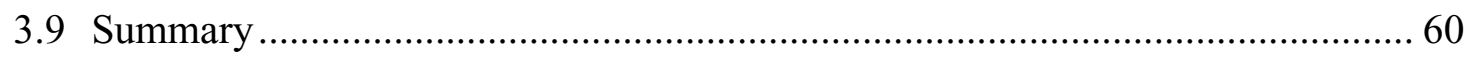

Chapter 4 - Analysis and Discussion ..........................................................62 62

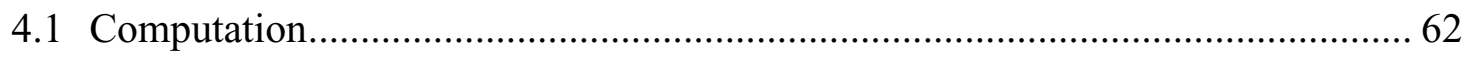

4.2 Impact of Truncating the Quasi-Renewal Function............................................. 65

4.3 Impact of Truncating the Point Availability Expressions......................................... 67

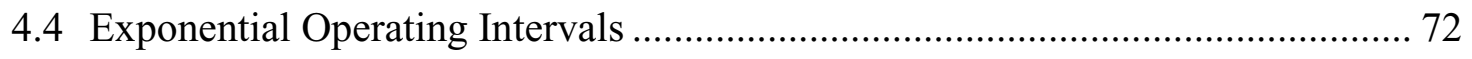

4.4.1 Exponential Quasi-Renewal Function......................................................... 73

4.4.2 Exponential Repair Intervals ................................................................ 73

4.5 Normally Distibuted Operating Intervals.......................................................... 76

4.5.1 Normal Quasi-Renewal Function .............................................................. 76

4.5.2 Normally Distributed Repair Intervals ...................................................... 79 


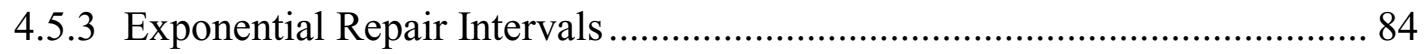

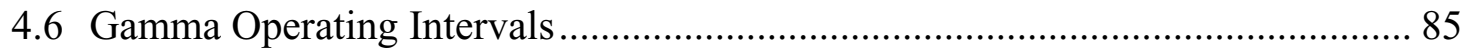

4.6.1 Gamma Quasi-Renewal Function ........................................................ 85

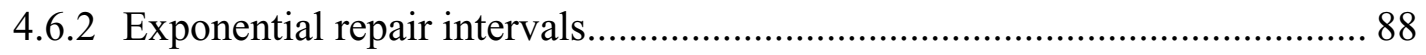

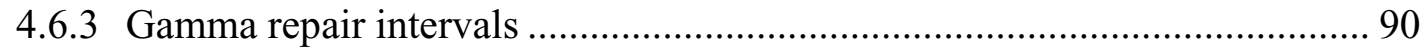

4.7 Compensation for Computational Limitations............................................ 92

Chapter 5 - Conclusions............................................................................... 100

Chapter 6 - Future Research...............................................................103

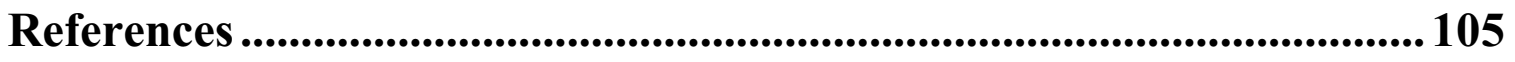

Appendix A - Sample Mathematica Input.................................................... 111

A.1 Exponential $(\lambda)$ Operating \& Exponential $(\mu)$ Repair ..................................... 111

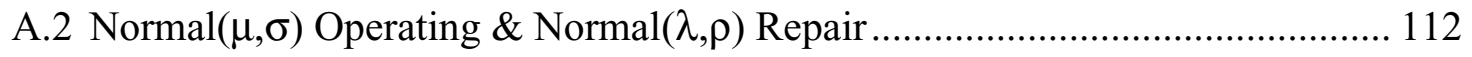

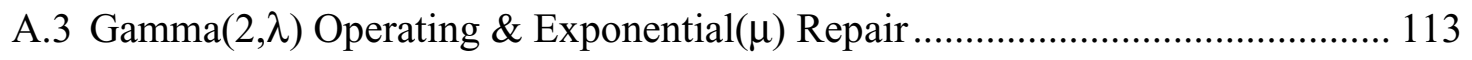

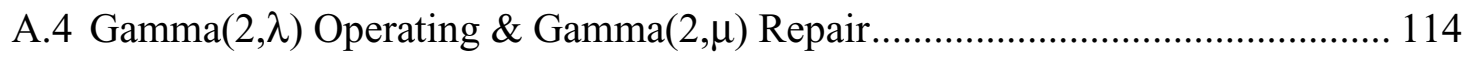

Appendix B - Sample Inverted Laplace Transforms .............................. 115

B.1 First 5 Terms for $\operatorname{Gamma}(2, \lambda)$ Operating \& Exponential $(\mu)$ Repair................. 115

B.2 First 5 Terms for $\operatorname{Gamma}(2, \lambda)$ Operating \& $\operatorname{Gamma}(2, \mu)$ Repair .................. 119

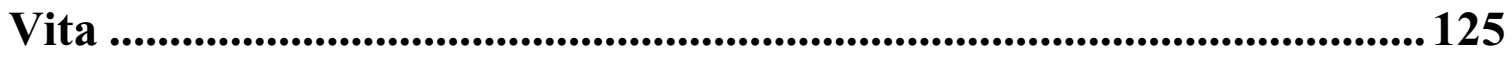




\section{List of Figures}

Figure 3.1 Graph of $F_{X_{1}}(t), F_{X_{2}}(t), F_{X_{3}}(t)$, and $F_{X_{4}}(t)$ for a quasi-renewal process $\ldots . .34$

Figure 3.2 Graph of $f_{X_{1}}(t), f_{X_{2}}(t), f_{X_{3}}(t)$, and $f_{X_{4}}(t)$ for a quasi-renewal process.... 34

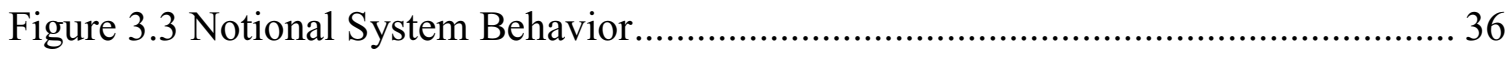

Figure 4.1 Normal $Q_{F}(t)$ with $\left.(\mu=100, \sigma=25, a=\{0.85,0.90,0.95,1.00)\}, c=20\right) \ldots \ldots 66$

Figure 4.2 Exponential $Q_{F}(t)$ with $\left.(\lambda=0.01, a=\{0.80,0.90,0.95\}), c=22\right) \ldots \ldots \ldots \ldots \ldots \ldots . . . . . .67$

Figure 4.3 Individual Terms for the lower bound on point availability.......................... 68

Figure 4.4 Individual terms for the upper bound on point availability .......................... 69

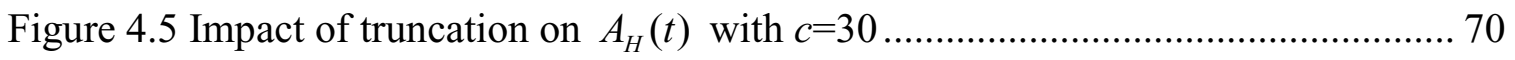

Figure 4.6 Upper bound used to determine error in approximation .............................. 71

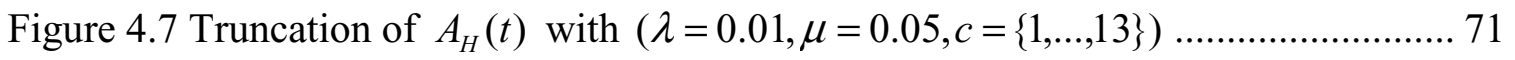

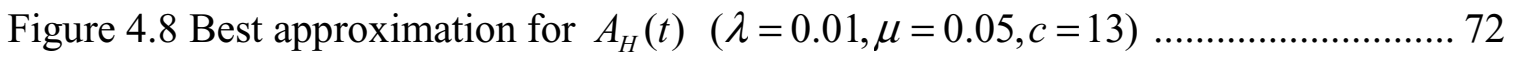

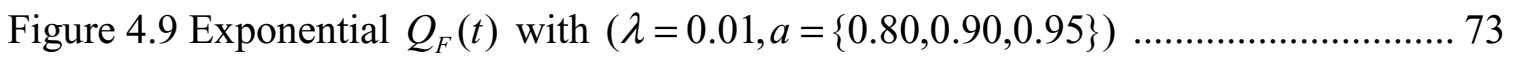

Figure 4.10 Exponential $A_{H}(t)$ with $(\lambda=0.01, a=\{0.85,0.90,0.95\}, \mu=0.10, b=1.1) \ldots 74$

Figure 4.11 Exponential $A_{H}(t)$ with $(\lambda=0.01, a=0.85, \mu=\{1 / 15,1 / 10,1 / 5\}, b=1.1) \ldots .75$

Figure 4.12 Exponential $A_{H}(t)$ with $(\lambda=0.01, a=0.9, \mu=0.10, b=\{1.0,1.1,1.2\}) \ldots \ldots . .75$

Figure 4.13 Normal $Q_{F}(t)$ with $(\mu=100, \sigma=25, a=\{0.85,0.90,0.95,1.00\}) \ldots \ldots \ldots \ldots \ldots \ldots . . .76$

Figure 4.14 Normal $Q_{F}(t)$ with $(\mu=100, \sigma=12.5, a=\{0.85,0.90,0.95,1.00\}) \ldots \ldots \ldots \ldots \ldots . .77$

Figure 4.15 Normal $Q_{F}(t)$ with $(\mu=100, \sigma=12.5, a=\{0.85,0.90,0.95,1.00\}) \ldots \ldots \ldots \ldots . .78$

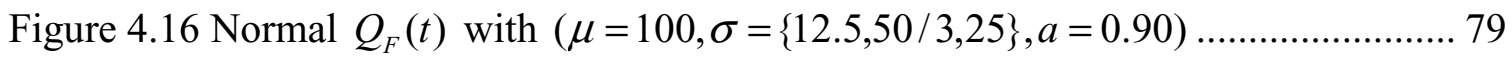

Figure 4.17 Normal $Q_{F}(t)$ with $(\mu=100, \sigma=25, a=0.90, \lambda=10, \rho=2.5, b=1.0) \ldots \ldots .79$

Figure 4.18 Normal $A_{H}(t)$ with

$$
(\mu=100, \sigma=\{12.5,50 / 3,25\}, a=0.90, \lambda=10, \rho=2.5, b=1.1)
$$

Figure 4.19 Normal $A_{H}(t)$ with

$$
(\mu=100, \sigma=25, a=\{0.85,0.90,0.95\}, \lambda=10, \rho=2.5, b=1.0)
$$


Figure 4.20 Normal $A_{H}(t)$ with

$$
(\mu=100, \sigma=25, a=0.90, \lambda=10, \rho=2.50, b=\{1.0,1.1,1.2\}) .
$$

Figure 4.21 Normal $A_{H}(t)$ with

$$
(\mu=100, \sigma=25, a=0.90, \lambda=\{5,10,15\}, \rho=2.5, b=1.1) .
$$

Figure 4.22 Normal $A_{H}(t)$ with

$$
(\mu=100, \sigma=25, a=0.90, \lambda=10, \rho=\{2.50,3.33,5\}, b=1.1)
$$

Figure 4.23 Gamma $Q_{F}(t)$ with $(k=2, \lambda=0.02, a=\{0.80,0.85,0.90\}) \ldots \ldots \ldots \ldots \ldots \ldots \ldots \ldots . . .86$

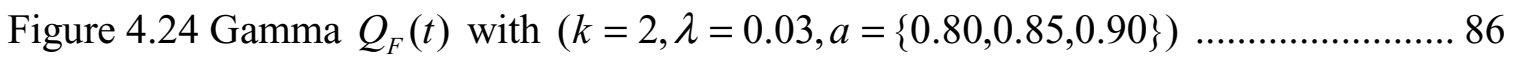

Figure 4.25 Gamma $Q_{F}(t)$ with $(k=3, \lambda=0.03, a=\{0.80,0.85,0.90\}) \ldots \ldots \ldots \ldots \ldots \ldots \ldots \ldots \ldots . . .87$

Figure 4.26 Gamma $Q_{F}(t)$ with $(k=\{2,3\}, \lambda=0.03, a=\{0.80,0.85,0.90\}) \ldots \ldots \ldots \ldots \ldots \ldots . . .87$

Figure 4.27 Gamma $A_{H}(t)$ with

$$
(k=2, \lambda=1 / 50, a=0.85, r=1, \mu=1 / 10, b=\{1.1,1.2,1.3\})
$$

Figure 4.28 Gamma $A_{H}(t)$ with

$$
(k=2, \lambda=1 / 50, a=\{0.75,0.80,0.85\}, r=1, \mu=1 / 10, b=1.1)
$$

Figure 4.29 Gamma $A_{H}(t)$ with

$$
(k=2, \lambda=1 / 50, a=0.85, r=1, \mu=\{1 / 5,1 / 10,1 / 15\}, b=1.1)
$$

Figure 4.30 Gamma $A_{H}(t)$ with

$$
(k=2, \lambda=2 / 100, a=\{0.80,0.85,0.90\}, r=2, \mu=2 / 10, b=1.1)
$$

Figure 4.31 Gamma $A_{H}(t)$ with

$$
(k=2, \lambda=2 / 100, a=0.85, r=2, \mu=2 / 10, b=\{1.1,1.2,1.3\})
$$

Figure 4.32 Gamma $A_{H}(t)$ with

$$
(k=2, \lambda=2 / 100, a=0.85, r=2, \mu=\{2 / 5,2 / 10,2 / 15\}, b=1.1) .
$$

Figure 4.33 Contribution of term 3 to $A_{H}(t)$ with $(\lambda=0.01, a=0.9, \mu=0.1, b=1.2) \ldots 94$

Figure 4.34 Contribution of term 3 to $A_{H}(t)$ with $(\lambda=0.01, a=0.9, \mu=0.1, b=1.2) \ldots 95$

Figure 4.35 Contribution of term 13 to $A_{H}(t)$ with $(\lambda=0.01, a=0.9, \mu=0.1, b=1.2) .95$

Figure 4.36 Contribution of term 13 to $A_{H}(t)$ with $(\lambda=0.01, a=0.9, \mu=0.1, b=1.2) .96$ 
Figure 4.37 Contribution of terms to $A_{H}(t)$ with

$$
(k=2, \lambda=1 / 50, a=0.85, r=1, \mu=0.1, b=1.1)
$$

Figure 4.38 Contribution of term 8 to $A_{H}(t)$ with

$$
(k=2, \lambda=1 / 50, a=0.85, r=1, \mu=0.1, b=1.0)
$$

Figure 4.39 Contribution of terms to $A_{H}(t)$ with

$$
(k=2, \lambda=1 / 50, a=0.85, r=1, \mu=0.1, b=1.1)
$$




\section{Chapter 1 - Introduction}

\subsection{Background}

As systems become more complex and automation increases, there is a growing interest in the study of equipment maintenance. Increased dependence on industrial systems, military systems, and computer networks has emphasized the importance of maintaining systems to reduce unplanned downtime and maintenance costs. For instance, all large-scale systems fielded by the U.S. Army must pass an evaluation of operational suitability, which is measured in terms of reliability and maintainability performance. Since the implications of unplanned failure and downtime can translate to the difference between life and death for soldiers, the Army has a structured multi-stage program to evaluate the suitability of its systems.

Since the early 1960 's, the academic community has shown a growing interest in maintenance optimization. McCall [1965], Pierskalla and Voelker [1976], and ValdezFlores and Feldman [1989] provide extensive surveys of the academic research conducted in the field of maintenance planning. Barlow and Proschan [1967, 1975, 1981] and Ascher and Fiengold [1984] are standard books that provide comprehensive coverage of basic concepts in reliability and maintainability. Nachlas and Cassady [1999] discuss the potential productivity benefits of maintenance planning and optimization. They assert that an increased emphasis on the study of equipment maintenance may lead to increases in competitive success that is similar to those that resulted from increased emphasis on quality improvement.

The goal of any maintenance program is to optimize system performance through cost reduction and increased availability. This is achieved by reducing the frequency of failures and the amount of downtime. In many situations the cost of a failure includes costs associated with bringing the system back to an operational state and the costs associated with the downtime incurred. Downtime consists of the time it takes to 
discover that a failure exists, identify the problem, acquire the appropriate tools and parts, and perform the necessary maintenance actions.

It is assumed the reader has an understanding of various basic concepts in the field of reliability and maintenance planning such as preventive maintenance, corrective maintenance, reliability, and availability. In addition, it is critical to have a strong foundation in probability theory and stochastic processes. An understanding of results from renewal theory, results from distribution theory, Poisson processes, and tools such as the Laplace transform are essential in the development of this dissertation. A brief overview of the different types of availability expressions and the formula for point availability as it is used in the context of a homogeneous process is provided below since the extension of these ideas under the assumption of a quasi-renewal process is the focus of this research. An explanation of some of the other terminology listed above is contained in Chapter 2.

There are four basic types of availability measures: point availability, limiting availability, average availability, and limiting average availability. All of these measures are related and can be defined in terms of the point availability, which is often simply referred to as the availability. The point availability, $A(t)$, is defined as the probability that the system is operational at any point in time, $t$. This is the availability measure that gives the most detailed description of system behavior. The limiting availability, $A$, of a system is the limit of $A(t)$ :

$$
A=\lim _{t \rightarrow \infty} A(t) .
$$

The average availability, $A_{\text {ave }}(t)$, over an interval $[0, \tau]$ is:

$$
A_{\text {ave }}(\tau)=\frac{1}{\tau} \int_{0}^{\tau} A(t) d t
$$

The average availability measures the fraction of time that the system is operational over the interval of interest. Even practitioners sometimes confuse the average availability with the point availability. The final availability measure is the limiting average availability, $A_{\infty}$, defined as follows: 


$$
A_{\infty}=\lim _{\tau \rightarrow \infty} A_{\text {ave }}(\tau)=\lim _{t \rightarrow \infty} \frac{1}{\tau} \int_{0}^{\tau} A(t) d t
$$

The expression for the point availability under the assumption of a basic homogeneous process is given by:

$$
A(t)=\bar{F}(t)+\int_{0}^{t} \bar{F}(t-x) m_{H}(x) d x
$$

where $F(t)$ is the distribution function on an interval of operation and $m_{H}(x)$ is the renewal density based on $H$ which is the distribution function on a single operationrepair cycle. The renewal density, $m_{H}(x)$, is the derivative of the renewal function, $M_{F}(t)$. For a homogeneous process with identically distributed lifetimes, $F(t)$, the renewal function is the distribution on the expected number of renewals at time $t$ and takes the form:

$$
\begin{aligned}
M_{F}(t) & =E[N(t)]=\sum_{n=0}^{\infty} n \operatorname{Pr}[N(t)=n] \\
& =\sum_{n=0}^{\infty} n(\operatorname{Pr}[N(t) \geq n]-\operatorname{Pr}[N(t) \geq n+1]) \\
& =\sum_{n=0}^{\infty} n\left(F^{(n)}(t)-F^{(n+1)}(t)\right) \\
& =\sum_{n=1}^{\infty} F^{(n)}(t)
\end{aligned}
$$

where $F^{(n)}(t)$ is the n-fold convolution of $F(t)$. Note that this development treats the instantaneous repair case. If repair times are included, the renewal function is based on $H(t)$, the convolution of $F(t)$ and the repair distribution, instead of $F(t)$ as in 1.4.

\subsection{Motivation}

Many people have modeled and analyzed various policies regarding the corrective maintenance, preventive maintenance, and availability of systems under the assumption of renewal after maintenance. The literature related to such homogeneous processes is extensive and key results can be found in the basic references cited above. 
A small portion of the literature addresses the situation in which renewal is not an appropriate assumption. These are imperfect repair models, or non-homogeneous processes (NHP), and are more realistic for situations encountered in practice. The minimal repair model is the most frequently discussed imperfect repair model in the literature and is a special case of an NHP. Specifically, the minimal repair model constitutes a non-homogeneous Poisson process (NHPP) where repair returns the system to its condition immediately prior to failure. The broader class of imperfect repair models consists of all models that allow repair actions to return the system to an intermediate condition between minimal repair and renewal. The homogeneous process is often a special case of an imperfect repair model. However, constructing models and obtaining analytical results under the assumption of an NHP is often relatively difficult. This is particularly true when developing an expression for the point availability of an NHP. As an alternative, the majority of the research focuses on the development of cost models that are based on the limiting behavior of a system.

Constructing a probability model of availability is an attempt to describe the actual behavior of a system, where a cost model analysis only yields optimal parameters under a particular cost structure. The importance of modeling the behavior of a system, rather than just the cost, is stressed in the above military example where life and death is at stake. In a less extreme industrial setting, an expression for the point availability can be used to predict and improve equipment utilization. For this reason, the lack of modeling and analysis of non-homogeneous processes that focus on availability, rather than cost, is unfortunate.

\subsection{Research Objectives}

The majority of imperfect repair models only consider some combination of minimal repair and renewal. However, Lam [1988] defines a non-homogeneous process called the quasi-renewal process that provides for the structured deterioration of both the operation and repair intervals over time, rather than restricting the possibilities to that of perfect repair or minimal repair. Wang and Pham [1996b, 1997] call this the quasi-renewal 
process and obtain the Laplace transform of the quasi-renewal function in terms of the transform of the first interval of operation. The focus is then shifted to constructing a cost model that is analyzed to determine the optimal length of a preventive maintenance interval. Wang and Pham make no attempt to construct a probability model that describes the behavior of the quasi-renewal process over time.

The goal of this research is to extend the work done by Wang and Pham through the development of an expression for the point availability of the quasi-renewal process under a series of increasingly more complex assumptions concerning the repair activity. It is assumed the system of interest can be modeled as a single component and that the system is monitored continuously and accurately. Therefore, the construction of an inspection policy is not an issue.

The construction of a probability model of equipment performance is a difficult task and it is often necessary to build such a model through an iterative process. The foundation for this analysis is a probability model that assumes instantaneous repair. An expression of the distribution of the expected number of failures as a function of time is a result of the first model. This result is the parallel to the basic renewal function of a homogeneous process with instantaneous repair. To develop the most basic availability model for the quasi-renewal process, repair times are included and assumed to be independent and identically distributed (iid). Finally, the repair times are assumed to increase stochastically according to a quasi-renewal process. The availability expressions for the above scenarios are solved analytically when possible, and numerically if obtaining an analytical solution is prohibitive.

\subsection{Results and Conclusions}

Exact expressions for the quasi-renewal function and the point availability function are found for the following cases

- exponential operating intervals and exponential repair intervals,

- normal operating intervals and normal repair intervals, 
- normal operating intervals and exponential repair intervals,

- gamma operating intervals and gamma repair intervals,

- gamma operating intervals and exponential repair intervals.

With the exception of the case of normal operating intervals and normal repair intervals, the exact expressions are infinite sums in the Laplace transform space. It is necessary to truncate the exact expressions so they can be inverted to the time domain to obtain numerical results. The truncation that is necessary causes the expressions in the time domain to be approximate.

Equations for the case of normal operating intervals and normal repair intervals are expressed in the time domain as infinite sums of normal cumulative distribution functions. The estimation of the normal cumulative distribution function introduces as small amount of error in the value of the approximations. Numerical results are provided for all of the above cases except the cases of normal operating intervals with exponential repair intervals. The form of the Laplace transform prevented inversion under these conditions. Issues related to the machine precision of complex algebraic manipulation made numerical evaluation of the expressions for the point availability and the quasirenewal function difficult in many cases. This is the limiting factor in the analysis and application of the models developed within.

Finally, two exact expressions are provided for the point availability in all cases. These two expressions are equivalent, but become distinct after truncation. The two expressions are useful as bounds on the point availability that become tighter as the number of evaluated terms increases. In addition, the bounds indicate the maximum error in point availability calculations and determine the point of truncation necessary to achieve a specified level of accuracy for the estimation of the point availability function at any time of interest. 


\section{Chapter 2 - Literature Review}

\subsection{Introduction}

This chapter provides an overview of the literature related to the maintenance and reliability of single-component systems. It should be noted that the word component is used to describe any system or subsystem that is modeled and evaluated as a single entity. Almost all systems consist of multiple components and sub-components that work together toward a common objective. For instance, a personal computer commonly consists of several components: a monitor, printer, keyboard, mouse, $\mathrm{CPU}$, hard-drive, and so on. Each of those components consists of tens to thousands of other components, such as capacitors, resistors, diodes, buttons, and wiring. It is often too difficult to obtain analytical reliability and maintainability results for multi-component systems. Therefore, most analytical models consider complex systems as a single entity that is either operational or failed. This approach allows the model to reflect the aggregate systemlevel behavior without incorporating the detailed relationships between numerous components. This dissertation focuses on systems that can be reasonably modeled as single component systems. From this point forward, the term component or system is used to describe a system of components that can be modeled as a single entity.

The majority of this review provides a comprehensive discussion of the models commonly known as corrective maintenance (CM) models. Corrective maintenance $(\mathrm{CM})$ is comprised of all maintenance actions performed in response to a system failure. Various corrective maintenance policies are described in terms of the degree to which the system is repaired. Replacement or renewal refers to the case in which the system is "as good as new" after repair. For instance, if an engine is replaced or rebuilt it is probably safe to assume that the engine is "as good as new." Minimal repair refers to the case in which the system is "as bad as old" after repair. For instance, if a spark plug is adjusted it is probably safe to assume that no significant improvement has been made and the engine is "as bad as old." The perfect repair model and the minimal repair model are the two most established CM models in the literature and rely on very rigid assumptions 
concerning the degree of component repair. In more recent literature, imperfect repair models and general repair models are addressed. Imperfect repair considers the case where a repair renders the system somewhere between "as bad as old" and "as good as new." This is a considerably more versatile and realistic approach for most situations encountered in practice.

In addition to corrective maintenance, which is performed as a reaction to failure, there is preventive maintenance (PM), which is performed to decrease the likelihood of an unplanned failure. Typically, the system is scheduled for PM during a period of time that provides the least disturbance in productivity. For instance, one might take a car in for a tune-up and oil change during the weekend. This is probably the most universally recognized form of PM. Failure to perform these tasks on a regular basis increases the chance of a broken fan belt, broken timing belt, or even an engine seizure while driving to work. Obviously, the minor inconvenience presented by putting the car in the shop is a much more attractive alternative than a catastrophic event on the highway. This simple example illustrates the motivation for a PM program.

There are two basic types of PM programs, block replacement and age replacement. A block replacement policy requires that PM actions be performed at regularly spaced time intervals. Changing the oil after every month is an example of a block replacement policy. An age replacement policy requires that PM actions be performed after a specified amount of accumulated use. Changing the oil after every 4,000 miles is an example of an age replacement policy.

PM is justified in situations where the failure rate of a system tends to increase over time and the cost of an unexpected failure is significantly higher then the cost of a scheduled PM action. PM and CM policies must both be considered as a part of any comprehensive maintenance plan for such systems. In fact, the more recent maintenance literature rarely addresses either PM or CM in isolation. Rather, most papers focus on a particular PM strategy while assuming a certain CM model or focus on a particular CM strategy while assuming a particular PM model. As a result, it would be difficult to 
provide a survey of $\mathrm{CM}$ literature without an introduction to the PM literature. Therefore, section 2.3 of this review covers basic PM models and provides several survey papers that serve as a good starting point for further investigation. Section 2.4 is a detailed overview of CM models, focusing on imperfect repair and general repair models. Finally, section 2.5 introduces the topic of component inspection.

Inspection models are tangential to this dissertation, but are contained in many of the papers related to PM and CM. Many models consider components that are monitored continuously and are subject to a continuous aging process. Inspection models and shock models consider components that are monitored at discrete points in time and components that are subject to a discrete aging process, respectively. In many situations it is impossible or cost prohibitive to continuously monitor the status of a component. For instance, the status of many safety systems is not observable without conducting a test or an inspection. A consequence of this situation is that upon failure the system remains in a failed state until the next inspection. The cost of inspection and the consequences of downtime must be considered to determine the optimal inspection policy for such systems.

The foundation of any maintenance model relies on the underlying deterioration process and failure behavior of the component. Therefore, following section gives a general description of some of the basic types of failure models.

\subsection{Failure Models}

Failure models are mathematical representations of the processes by which a component deteriorates and ultimately fails. A CM or PM model is structured around various assumptions regarding the underlying failure process. A PM program is warranted if a component experiences significant deterioration over time. The factors that determine the actual instance of failure and the deterioration behavior influence the type of CM program that may be reasonable. 
It is common to model the aging process of a component as a continuous function over time. There are a number of classifications for such lifetime distributions, the most common of which are: increasing failure rate (IFR), decreasing failure rate (DFR), and constant failure rate (CFR). In choosing lifetime distributions or failure rate functions, it is often assumed that the aging process can be modeled with a number of theoretical distributions, without much concern for the physical implications of the parameter selection. However, three types of models focus more on the physical dynamics behind failure behavior: shock models, Brownian motion models, and shot-noise models.

A component that tends to age as the result of a series of distinct stresses or shocks at discrete points in time is described with a shock model. The frequency and magnitude of the shocks are often modeled with various probability distributions. Failure of such a component occurs at a point in time when the cumulative damage from preceding shocks has exceeded a threshold. The frequency of shocks is commonly modeled as a Poisson or non-homogeneous Poisson process. A discussion of recent shock models can be found in Valdez-Flores and Feldman [1989] and Pham and Wang [1996]. Early shock models, described in Esary, Marshall, and Proschan [1973] and Ross [1981], assume that the intervals between shocks are exponentially distributed and are called Poisson shock models. Generally, a component must be repaired or replaced after the cumulative damage from all of the shocks has reached a certain threshold. Shanthikumar and Sumita [1983] develop a shock model where the magnitude of a shock is correlated with either the length of the interval since the previous shock, or the length of the interval until the next shock. A model described by Kijima and Nakagawa [1992] specifies the probability of component failure as a function of cumulative damage, as opposed to a pre-determined constant threshold. The situation where PM is imperfect is considered. A cost model is developed to determine both the optimal number of PM actions before replacement, and the optimum replacement interval.

The exponential time between shocks is relaxed by Rangan and Sarada [1993], who model the shock arrival process as a non-homogeneous Poisson process (NHPP). Shue [1998] evaluates the optimal block replacement time under the assumption of an NHPP 
shock model with two types of failure. Minor failures are minimally repaired and catastrophic failures are remedied with replacement of the component.

Lemoine and Wenocur [1985] provide an excellent discussion of two basic types of failure models, failure models based on Brownian motion and failure models based on a shot-noise process. The shot-noise model is similar to the shock models described above. In addition, they propose possible connections between common theoretical models used in practice and the underlying physical dynamics that cause observed failure behaviors. The Brownian motion model is built on the assumption that component strength $\mathrm{x}$ evolves according to a diffusion process $X=\{X(t), t \geq 0\}$ that has drift parameter $\mu(x)$ and diffusion coefficient $\sigma^{2}(\mathrm{x})$. Another aspect of the model is the "killing rate," $\mathrm{k}(\mathrm{x})$. This killing rate is the rate at which shocks that are of sufficient magnitude to cause a component of strength $\mathrm{x}$ to fail occur. Under the assumption that $\sigma^{2}(\mathrm{x})=0$, the stochastic process that describes the strength of the component reduces to:

$$
\mathrm{X}(\mathrm{t})=\int_{0}^{\mathrm{t}} \mu[\mathrm{X}(\mathrm{s})] \mathrm{ds}+\mathrm{x} .
$$

This expression is used to derive the following relationship between the killing rate function and the commonly used failure rate function, $r(t)$ :

$$
r(t)=\frac{f(t)}{\bar{F}(t)}=k[X(t)]
$$

where $F(t)$ is the life distribution of the component. This relationship is used to describe a possible physical interpretation of various parameters in several theoretical distributions used in modeling failure behavior, such as the Weibull and Rayleigh distributions.

The shot-noise model, discussed by Lemoine and Wenocur [1985, 1986], is based on the assumption that wear or aging takes place because of shots or jolts to the component. A component fails if the accumulated wear exceeds a threshold or if a shot of fatal magnitude occurs. The rate at which fatal shots occur is a function of the accumulated wear of the component. The shot-noise process $X=\{X(t), t \geq 0\}$ represents the operational age of the component. Shots takes place according to a Poisson process with a deterministic rate function. If the component survives a shot, it will recover to a certain 
degree with the passage of time. For example, if a shot of magnitude D occurs at time $S$, then the impact of the shot at time $\mathrm{S}+\mathrm{t}$ is $\mathrm{Dh}(\mathrm{t})$ where $\mathrm{h}$ is non-negative and decreasing in t. Therefore, $\mathrm{h}(\mathrm{t})$ models the rate of recovery, which is often assumed to follow an exponential distribution. If $\left\{\mathrm{S}_{\mathrm{n}}, \mathrm{n} \geq 1\right\}$ are the instances of shot occurrence and $\left\{D_{n}, n \geq 1\right\}$ are the associated magnitudes of the shots, then $X(t)$ is given by:

$$
X(t)=\sum_{n=1}^{\infty} D_{n} h\left(t-S_{n}\right) .
$$

\subsection{Preventive Maintenance}

For the past thirty years, there has been an increasing interest in the study of the preventive maintenance of components. McCall [1965] and Pierskalla and Voelker [1976] provide surveys of early preventive maintenance models. In addition, a comprehensive bibliography for the same period is contained in Osaki and Nakagawa [1976]. Subsequent papers by Sherif and Smith [1981], Valdez-Florez and Feldman [1989], and Murdock [1995] provide a more current summary of the existing literature.

\subsubsection{Age Replacement}

A component that is maintained under an age replacement policy is replaced after failure or at a specified operational age $\mathrm{T}$. The time required to replace the failed component is often considered negligible and, after replacement, the component is assumed to be "as good as new." These two assumptions allow basic renewal theory results to be used to describe component behavior. The relaxation of the renewal assumption in repair operations is the focus of ongoing research and will be addressed later in this chapter. A description of the above age replacement scheme and renewal theory results can be found in Barlow and Proschan [1967, 1975].

The choice of the optimal replacement age, $\mathrm{T}^{*}$, is the main problem in the study of age replacement policies. Ascher and Feingold [1984] review numerous cost models that have been developed to determine the optimal replacement age. If repair and 
replacement times are considered non-negligible, it is possible to construct models to determine the optimal replacement age in order to maximize component availability. Nachlas [1989] develops a cost optimization model that incorporates a constraint on the width of the confidence interval on availability. Murdock [1995] conducted research to model the situation in which distinct distributions on the time required for PM and the time required for $\mathrm{CM}$ exist. This model allows the calculation of an age replacement time that maximizes interval availability. Subsequently, Reineke, Murdock, Pohl, and Rehmert [1999] extend this research with models that optimize either the limiting availability or the cost rate of complex systems. Insight is offered into the evaluation of the tradeoffs between the two models. Cassady, Maillart, Bowden, and Smith [1998] construct a model to approximate the replacement age that maximizes the average availability over the useful life of a component. Experimental design and regression analysis are used to find a first order approximation of the functional relationship between lifetime and repair time parameters and the optimal replacement age.

\subsubsection{Block Replacement}

A component or system that is maintained under a block replacement policy is replaced at regular time intervals $(\mathrm{T}, 2 \mathrm{~T}, 3 \mathrm{~T}, \ldots, \mathrm{kT})$ regardless of age. The block replacement policy is easier to administer than an age replacement policy since only the elapsed time since the last replacement must be monitored, rather than the operational time since the last replacement. Cox [1962] defines cost models for both age and block replacement policies and indicates that an age replacement policy is preferable to a block replacement policy. Under a block replacement policy, a component that was just replaced after failure may be replaced again as a part of the planned block replacement. In addition, Cox specifies conditions that are necessary for age and block replacement policies to be preferable over simple corrective maintenance.

Assuming an increasing failure rate (IFR) life distribution, Barlow and Proschan [1975] prove that a block replacement policy will lead to fewer failures than an agereplacement policy. However, the block replacement policy will require more 
replacements than an age-replacement policy. When choosing between traditional age and block replacement policies, the cost of increased premature replacement must be balanced with the cost of increased failures and the increased cost of monitoring the operational age of a component.

Several modified block replacement policies have been introduced in order to reduce the cost associated with the premature replacements inherent in a traditional block replacement policy. All of these modified policies still benefit from the fact that preventive maintenance actions are scheduled at regular time intervals. Barlow and Hunter [1960] develop a block replacement model where components are replaced at time $\mathrm{T}$ and are only minimally repaired upon failure. The assumption is that a minimal repair is often adequate until the next planned replacement. The concept of minimal repair is discussed in detail in section 2.4.2. Cox [1962] formulates a model to reduce the number of premature replacements in the block replacement policy by allowing a component to remain in a failed state if it fails in the interval $\left[(k-1) T+T_{0}, k T\right]$. More recently, Sheu [1996] combines these ideas and suggests that if a component fails in the interval $\left[(\mathrm{k}-1) \mathrm{T},(\mathrm{k}-1) \mathrm{T}+\mathrm{T}_{0}\right]$ it is either replaced or minimally repaired, and if it fails within the interval $\left[(\mathrm{k}-1) \mathrm{T}+\mathrm{T}_{0}, \mathrm{kT}\right]$ it is either minimally repaired or remains in a failed state until kT. Similar block replacement models can be found in Berg and Epstein [1976], Tango [1978], Shue [1994], and Murthy and Nguyen [1982]. Various renewal theory results concerning block replacement policies are detailed and summarized in Shaked and Zhu [1992].

\subsubsection{Imperfect Preventive Maintenance}

Several authors have explored the situation in which preventive maintenance actions do not completely renew a component. Lie and Chun [1986] consider the case in which there are two types of preventive maintenance actions, simple PM and preventive replacement. Under a simple PM action, the failure rate of the component is reduced to a level between the failure rate just prior to failure and that of a new component. This type of simple PM is also discussed by Malik [1979] and is called the improvement factor 
model. Preventive replacement provides complete renewal of the component through replacement. The average cost rate per cycle is minimized to determine the optimal number of simple PM actions performed before a preventive replacement. This model also provides for two types of corrective maintenance actions, minimal repair and corrective replacement. Corrective maintenance actions are discussed in the next section.

A shock model with imperfect preventive maintenance is developed by Kijima and Nakagawa [1992]. Shocks to the component arrive according to a Poisson process and each shock causes a component failure with a probability that is a function of the total accumulated damage from previous shocks. A series of imperfect PM actions are performed until the $\mathrm{N}^{\text {th }} \mathrm{PM}$ action, which is a replacement. If the accumulated age in the $\mathrm{k}^{\text {th }}$ period of operation is $\mathrm{Y}_{\mathrm{k}}$, then repair reduces the accumulated age in the $\mathrm{k}^{\text {th }}$ period to $b_{k} Y_{k}$ for $0 \leq b<1$. A cost model is constructed to determine the optimal value of $\mathrm{N}$ for a fixed PM interval, the optimal PM interval for a fixed value of N, and the optimal pair of $\mathrm{N}$ and PM interval.

Chan and Shaw [1993] define the concept of stochastic cycle availability as the proportion of "up time" during the time until renewal or replacement is necessitated by failure. A model is developed to maximize the probability of obtaining a high value for this measure under imperfect $\mathrm{PM}, \mathrm{P}\left[\mathrm{A}_{\mathrm{c}} \geq 1-\varepsilon\right]$ where $\mathrm{A}_{\mathrm{c}}$ is the cycle availability. The optimal value for the time between PM actions is found under two different assumptions concerning the impact of PM on the failure rate. First, each PM action reduces the failure rate by a constant amount as discussed above. The second model allows each PM action to reduce the failure rate by an amount that is proportional to the failure rate just prior to failure. The failure rate is assumed to follow a Weibull distribution with shape parameter $\alpha=2$. The failure rate for both models is expressed as follows: $r_{n}(t)=\alpha \lambda(\lambda t)^{\alpha-1}-\Delta_{n}$ for $t \in\left[t_{n-1}, t_{n}\right)$, where $r_{n}(t)$ is the failure rate at time $t$ that falls in the interval between the $(\mathrm{n}-1)^{\text {th }}$ and $\mathrm{n}^{\text {th }} \mathrm{PM}$ before replacement, and $\Delta_{\mathrm{n}}$ is the cumulative reduction in failure rate as a result of PM. For the fixed reduction model $\Delta_{\mathrm{n}}=\Delta_{\mathrm{n}-1}+\mathrm{g}$, where $\mathrm{g}$ is the constant reduction amount. For the proportional reduction $\Delta_{\mathrm{n}}=\Delta_{\mathrm{n}-1}+\mathrm{g} \mathrm{r}_{\mathrm{n}-1}\left(\mathrm{t}_{\mathrm{n}-1}\right)$, so the reduction 
in failure rate is a proportion, $g$, of the current failure rate. Numerical results are obtained for $\mathrm{g}=0.5$, and $\mathrm{P}\left[\mathrm{A}_{\mathrm{c}} \geq 0.9\right]$ is plotted against the time between $\mathrm{PM}$ actions for the fixed reduction model, the proportional reduction model, and the case where there is no PM. The optimal PM interval is identified for each of the two failure reduction models.

Wang and Pham [1996a] discuss two imperfect PM models that are based on the concept that the time between failures is stochastically decreasing by a factor $\alpha$ and repair times are stochastically increasing by a factor $\beta$, until the $\mathrm{k}^{\text {th }}$ repair. Both models assume that PM actions are not scheduled until after the $\mathrm{k}^{\text {th }}$ repair. After the $\mathrm{k}^{\text {th }}$ repair, PM is performed at regular intervals of length $\mathrm{T}$ and failures are minimally repaired. In the first model PM is imperfect in the sense that the component is renewed with probability $\mathrm{p}$ and is unchanged with probability $\mathrm{q}=1-\mathrm{p}$. This is similar to the $(\mathrm{p}, \mathrm{q})$ rule for imperfect $\mathrm{CM}$ that is discussed below. A long run expected cost equation and an asymptotic average availability equation are constructed. The cost equation is used to determine the unconstrained optimal values of $\mathrm{T}$ for a fixed $\mathrm{k}$, and the optimal values for both $\mathrm{k}$ and $\mathrm{T}$. The asymptotic average availability equation is used in a constrained optimization model to determine the minimum cost given a minimum availability, and the maximum availability given a maximum cost. It is important to note that the minimal repairs are assumed to occur in a negligible amount of time, therefore only the perfect PM actions impact the availability calculations. The second imperfect PM model assumes that a PM action makes a component $\mathrm{x}$ units of time younger. The value $\mathrm{x}$ is a constant such that $0 \leq x \leq T$. This is similar to the additive age model for general repair discussed below. The component is replaced after N PM actions. The expected replacement time and the expected PM times are distinct. A cost model is used to determine the optimal values of $\mathrm{N}, \mathrm{T}$, and $\mathrm{k}$.

\subsection{Corrective Maintenance}

Although many of the papers cited provide models that address both topics, the emphasis of the previous section was on preventive maintenance, as opposed to corrective maintenance. Corrective maintenance actions are those actions necessary to 
restore components to an operational state after failure, and can be categorized as follows: perfect repair, minimal repair, imperfect repair, and general repair. In the above categories, repair may be used interchangeably with replacement. The issue is not whether a repair or replacement takes place. Rather, the issue is the relative age of the component after repair or replacement. For example, if a failed component is replaced by a new component, it is considered the same as if the component was repaired to an "as good as new" condition.

Nachlas and Cassady [1999] assert that there is an optimal balance between preventive maintenance actions and corrective maintenance actions. Increased PM will reduce the number of $\mathrm{CM}$ actions required, however the growing cost of an aggressive PM policy will begin to outweigh the savings in $\mathrm{CM}$ actions at some point. As mentioned above, the evaluation of PM and CM policies must be considered simultaneously in order to maximize overall system performance. Many of the papers cited in this chapter address both PM and CM strategies, but focus more on the decisions regarding one or the other.

\subsubsection{Perfect Repair}

Perfect repair models assume that after a corrective maintenance action the component is rendered "as good as new." The perfect repair assumption is reasonable if failed components are replaced by new and identical components or if the repair procedure is thorough enough to negate nearly all of the aging effects. Models that assume perfect repair are able to take advantage of basic renewal theory and queueing theory results contained in Barlow and Proschan [1975], Cox [1962], Daigle [1992], Clarke and Disney [1985], and many other textbooks on the subject. Many current models assume perfect repair at some point for analytical tractability. However, models that rely heavily on the perfect repair assumption have been well established in the literature as mentioned above. 
Some of the shortcomings of using a perfect repair or renewal model are discussed in Ascher and Feingold [1984]. If the modeled system actually represents a number of components, then the repair or replacement of any one component will rarely renew the entire system. The remaining components that have not failed have accumulated wear due to an aging process. In addition, the repair of one component is not always completely performed and other components may be damaged in the repair effort. Economically, it may be unreasonable to perform a complete repair or replacement at every failure. Commonly, components are repaired to a state that is likely to hold the system operational until the next overhaul, or a component may be replaced by a used component rather than a new component. Minimal repair models were some of the first models to address the fact that alternatives to the perfect repair model are necessary.

\subsubsection{Minimal Repair}

The concept of minimal repair was first introduced by Barlow and Hunter [1960] and discussed again in Barlow and Proschan [1967]. The survey paper by Valdez-Flores and Feldman [1989] devotes a section to the discussion of minimal repair models. Under minimal repair, a component is repaired to a state that is stochastically identical to the state just prior to failure. In other words, the failure rate is identical before and after a maintenance action. Justification for the minimal repair assumption is commonly expressed in one of two ways. First, a conscious decision may be made to repair a system just enough to restore operation in an effort to avoid the time or material required to renew the system. Second, the system may actually represent a large number of components. As mentioned above, the repair or replacement of a single failed component may have little effect on the state of the system when the state of all other components has not been altered.

Barlow and Hunter's model is defined in the context of a block replacement policy. A component is replaced at regular intervals, $\mathrm{kT}$, and any failures are minimally repaired. Several minimal repair models are constructed by Sandve and Aven [1999] to minimize the long run expected cost per time unit under three PM policies. The first policy is a 
traditional block replacement policy. The second policy allows for replacement instead of minimal repair if a component failure occurs close enough to the planned replacement time. Finally, the third policy requires a decision to be made at each component failure. Rather than using an age or block replacement framework, Shue [1993] proposes a model that determines the optimal number of minimal repairs until replacement. The cost model used expresses costs as a function of component age.

Several synonyms for minimal repair exist in the literature such as: "bad-as-old" Ascher [1968], "restoration process" Bassin [1969], and "age-persistence" Balaban [1978]. In a minimal repair policy, failures occur according to a non-homogeneous Poisson Process (NHPP). A counting process $\{\mathrm{N}(\mathrm{t}), \mathrm{t} \geq 0\}$ is said to be an NHPP if:

$$
\begin{aligned}
& \mathrm{N}(0)=0, \\
& \{\mathrm{~N}(\mathrm{t}), \mathrm{t} \geq 0\} \text { has independent increments, and } \\
& \mathrm{P}\left[\mathrm{N}\left(\mathrm{t}_{2}\right)-\mathrm{N}\left(\mathrm{t}_{1}\right)=\mathrm{n}\right]=e^{-\int_{\mathrm{t}_{1}}^{\mathrm{t}_{1}} \lambda(\mathrm{t}) \mathrm{dt}} \frac{\left[\int_{\mathrm{t}_{1}}^{\mathrm{t}_{2}} \lambda(\mathrm{t}) \mathrm{dt}\right]^{\mathrm{n}}}{\mathrm{n} !},
\end{aligned}
$$

where $\lambda(t)$ is the time-dependent rate of the process. A discussion of the properties and implications of a NHPP can be found in textbooks such as Ascher and Feingold [1984] and Barlow and Proschan [1967, 1975]. Some important technical notes regarding characteristics of the NHPP are offered by Murthy [1991] and Engelhardt and Bain [1986]. The expression for the expected number of failures in an interval is provided in Barlow and Hunter [1960] and has been used frequently in the literature. Murthy [1991] develops an expression for the cumulative distribution function (CDF) of the number of failures over an interval for the minimal repair model. A common reliability measure for HPP models is the mean time between failures (MTBF), Engelhardt and Bain [1986] explore the question of whether there is a reasonable alternative for the NHPP. They suggest the use of the reciprocal of the intensity function. The minimal repair model was the first step taken in the much broader context of imperfect maintenance models. 


\subsubsection{Imperfect Repair}

In the literature, the term imperfect repair has taken a broad meaning. Some authors have used imperfect repair synonymously with minimal repair, while most authors have defined imperfect repair to include policies with some mixture of minimal repair and perfect repair. More recently, general repair models have been discussed as the most generally applicable corrective maintenance model that includes perfect repair and minimal repair as special cases. General repair models are discussed in the next section.

A very thorough review of the literature concerning imperfect maintenance can be found in Pham and Wang [1996]. This survey addresses both imperfect preventive maintenance and corrective maintenance. It is important to note that, with the exception of Wang and Pham [1996a, 1996b, 1997], there have been no imperfect repair models that have attempted to use component availability as a performance measure instead of cost. In order to calculate availability the assumption that repair times are negligible must be eliminated. This assumption exists in most of the papers discussed in this review.

The first imperfect repair model is developed by Brown and Proschan [1983] and is referred to as the (p,q) model. The $(\mathrm{p}, \mathrm{q})$ model is built on the assumption that upon failure a component undergoes perfect repair with probability $\mathrm{p}$, and undergoes minimal repair with probability $\mathrm{q}=1-\mathrm{p}$. The distribution on the time between perfect repairs is given and is shown to preserve certain characteristics of the original life distribution of the component. The failure rate of the $(\mathrm{p}, \mathrm{q})$ model is shown to be a multiple, $\mathrm{p}$, of the original failure rate. Wang and Pham [1996a] use the (p,q) rule to model imperfect PM as mentioned above. This basic model is extended by Block, Borges, and Savits [1985] with the expression of the values $\mathrm{p}$ and $\mathrm{q}$ as a function of the age of the component, and is referred to as the $(\mathrm{p}(\mathrm{t}), \mathrm{q}(\mathrm{t}))$ model. Again, the distribution on the time between perfect repairs is given along with the associated failure rate. In both models, repair times are assumed negligible and availability results cannot be obtained. Iyer [1992] assumes that repair times for the $(\mathrm{p}(\mathrm{t}), \mathrm{q}(\mathrm{t}))$ model are non-negligible and obtains steady-state 
availability and down-time results. Minimal repair times are assumed independent and identically distributed and perfect repair times are assumed independent and identically distributed. The distribution on the number of minimal repairs until a perfect repair is developed. This distribution is used in conjunction with the distribution on the minimal repair time to determine the distribution on the time of the failure prior to perfect repair. The failure time prior to perfect repair and the time of completion of a perfect repair are renewal points of an alternating renewal process. The preceding results are used along with the mean perfect repair time, $\mu_{Y}$, to determine the long run fraction of time the component is up and available, down due to minimal repair, and down due to perfect repair. For instance, the steady state availability is given by:

$$
\text { Availability }=\frac{\mu(\mathrm{p})}{\mu(\mathrm{p})+\mu_{\mathrm{X}} \mu_{\mathrm{N}-1}+\mu_{\mathrm{Y}}},
$$

where $\mu(\mathrm{p})$ is the mean time until the first perfect repair, $\mu_{\mathrm{X}}$ is the mean minimal repair downtime, and $\mu_{\mathrm{N}-1}$ is the expected number of minimal repairs until a perfect repair. Shaked and Shanthikumar [1986] followed by Sheu and Griffith [1992] extend these models to the multivariate case where several components have dependent life-lengths.

Lam [1988] defines a geometric process as an alternative to the NHPP in modeling a component with stochastically decreasing lifetimes and stochastically increasing repair times. A sequence of random variables $\left\{\mathrm{X}_{1}, \mathrm{X}_{2}, \mathrm{X}_{3}, \ldots\right\}$ is a geometric process if for $\mathrm{a}>0$ the sequence $\left\{a^{n-1} X_{n}, n=1,2, \ldots\right\}$ forms a renewal process. For instance, if $a=2$ and $\left\{\mathrm{X}_{1}, 2 \mathrm{X}_{2}, 4 \mathrm{X}_{3}, \ldots\right\}$ forms a renewal process, then the $\mathrm{X}_{\mathrm{i}}^{\prime} \mathrm{s}$ must be stochastically decreasing as the index increases. The lifetimes are modeled with a non-increasing geometric process, $\mathrm{a} \geq 1$, and the repair times are modeled with a non-decreasing geometric process, $a \leq 1$. A traditional age replacement policy and a policy that specifies the number of failures until replacement are considered. The long-run average cost is used to determine the optimal parameters for both policies. Lam [1990] extends this work to a more general case and considers the policy where the optimal number of failures before replacement is determined. The restriction that the repair times and operation times constitute independent geometric processes is relaxed. The new 
restriction is that the expectation of successive repair times is non-decreasing and the expectation of successive operation times is non-increasing. Using a repair cost rate, a replacement cost, and a reward rate, the long-run average reward per unit time is defined and used as a performance measure to calculate the optimal number of repairs before replacement.

Finkelstein [1993a] develops a very similar model that has potential for use in the general repair case. First, a general deteriorating renewal process (GDRP) is defined such that $\mathrm{F}_{\mathrm{i}+1}(\mathrm{x}) \leq \mathrm{F}_{\mathrm{i}}(\mathrm{x})$ where $\mathrm{F}_{\mathrm{i}}(\mathrm{x})$ is the survivor function during the $\mathrm{i}^{\text {th }}$ interval of operation. The GDRP is modified so that the intensity of deterioration in each interval is described with a series of coefficients, $\mathrm{a}_{\mathrm{i}}$. This more specific model is called the particular deteriorating renewal process $(P D R P)$ and is defined such that $F_{i}(x)=F\left(a_{i} x\right)$ and $1=a_{1} \leq a_{2} \leq a_{3} \leq \ldots \leq a_{n}$. The PDRP has potential for use in a general repair model. However, Finkelstein provides a more restrictive geometrical PDRP (GPDRP) that predetermines the intensity of deterioration from any operational interval to the next. The GPDRP requires that $a_{i}=a^{i-1}$ so that the distribution on the length of the $i^{\text {th }}$ interval of operation is given by $F_{i}(x)=F\left(a^{i-1} x\right)$ where the value of $a$ is constant, $a>1$. The GPDRP is the focus of the remainder of the paper where renewal equations are developed and a cost model is formulated to determine the optimal value for the level of deterioration after each repair. Using the expected repair time and a cost per unit of repair time, a cost model that considers non-negligible repair times is also proposed.

Wang and Pham [1996b, 1997] refer to the same process described by Lam [1988] as a quasi-renewal process where successive interarrival distributions are scaled by a constant factor, $\alpha>0$. If we let $\left\{\mathrm{X}_{1}, \mathrm{X}_{2}, \mathrm{X}_{3}, \ldots\right\}$ represent the sequence of interarrival times of failures and let $\mathrm{Z}_{\mathrm{i}}$ be iid random variables, then $\left\{\mathrm{X}_{1}=\mathrm{Z}_{1}, \mathrm{X}_{2}=\alpha \mathrm{Z}_{2}, \mathrm{X}_{3} \alpha^{2} \mathrm{Z}_{3}, \ldots\right\}$ forms a quasi-renewal process. Characteristics of the interarrival density functions are discussed. In addition, the distribution on the number of failures over time and the renewal density are constructed. Three models are considered. The first model is a traditional block replacement policy and the optimal value for the replacement time is 
found under the assumption of negligible repair times. Corrective maintenance is performed between PM actions according to the quasi-renewal process. The second model is the same as the first model except that imperfect PM is assumed. Each PM action is assumed to renew the component with probability $\mathrm{p}$ and provide minimal repair with probability 1-p. For the first two models, it is shown that there is an optimal PM interval that minimizes the maintenance cost per unit time. The third model is based on the assumption of stochastically increasing repair times and the same quasi-renewal behavior up to the time of the $\mathrm{k}^{\text {th }}$ failure. After the $\mathrm{k}^{\text {th }}$ failure, $\mathrm{PM}$ is performed at regular intervals of length T. PM times are identically distributed with mean $w$ and corrective maintenance takes place in a negligible amount of time. A cost model is developed to determine the optimal PM interval, T, and the optimal number of failures before PM, k. The limiting average availability for the third model is expressed as a function of $\mathrm{k}$ and $\mathrm{T}$ :

$$
\mathrm{A}(\mathrm{T}, \mathrm{k})=\frac{\frac{\mu_{1}\left(1-\alpha^{\mathrm{k}-1}\right)}{1-\alpha}+\frac{\mathrm{T}}{\mathrm{p}}}{\frac{\mu_{1}\left(1-\alpha^{\mathrm{k}-1}\right)}{1-\alpha}+\frac{\eta_{1}\left(1-\beta^{\mathrm{k}-1}\right)}{1-\beta}+\frac{\mathrm{T}}{\mathrm{p}}+w} .
$$

The notation is as follows: $\mu_{1}$ is the mean of the first interval of operation, $\eta_{1}$ is the mean of the first repair time, and $\beta$ is the factor by which repair times increase until the $\mathrm{k}^{\text {th }}$ failure.

Three types of maintenance actions, minimal repair, periodic overhaul, and complete renewal are considered in the model developed by Zhang and Jardine [1998]. The effects of maintenance actions are modeled through changes in the failure rate of the component. Minimal repair leaves the failure rate unchanged, a periodic overhaul returns the failure rate to a point between the failure rate at the previous overhaul and minimal repair, and renewals are performed according to an age replacement policy. Minimal repairs are performed at failures. The degree of repair achieved with an overhaul is assigned the value $\mathrm{p}$ and is constant for every overhaul performed. Therefore, $\mathrm{v}_{\mathrm{k}}(\mathrm{t})=\mathrm{pv}_{\mathrm{k}-1}(\mathrm{t}-\mathrm{s})+(1-\mathrm{p}) \mathrm{v}_{\mathrm{k}-1}(\mathrm{t})$, where $\mathrm{v}_{\mathrm{k}}(\mathrm{t})$ is the failure rate at time $\mathrm{t}$ and after the $\mathrm{k}^{\text {th }}$ overhaul, and $\mathrm{s}$ is the time between overhauls. Cost models are developed to determine 
the optimal time between overhauls and the optimal number of overhauls per renewal cycle.

\subsubsection{General Repair}

General repair models are actually the most widely applicable imperfect repair models and include perfect repair and minimal repair as special cases. These models are discussed independently of imperfect repair models since general repair models are particularly relevant to this dissertation and are investigated in detail. Most of the models discussed in the sections concerning imperfect repair and imperfect preventive maintenance can be extended to the general repair case. The distinct feature of a general repair model is that every repair effort can render the state of the component anywhere in the continuum from just prior to failure to complete renewal. Some general repair models provide for the cases in which repair efforts potentially make the state of the component worse than just prior to failure, or better than renewal. These characteristics of general repair models are what separate the following models from those discussed in the imperfect repair section.

\subsubsection{Virtual Age Model}

The concept of virtual age was first introduced by Kijima, Morimura, and Suzuki [1988]. This model allows repair actions to bring the state of the component to a value somewhere between completely new and just prior to failure. Replacements are made according to a block replacement policy. Let $V_{n}$ designate the virtual age of the component immediately after the $n^{\text {th }}$ repair, $X_{n}$ designate the time between the $(n-1)^{\text {th }}$ and $\mathrm{n}^{\text {th }}$ repair, and $\mathrm{F}(\mathrm{x})$ designate the life distribution of a new component. The time between repairs depends on the virtual age after the most recent repair and is distributed as follows:

$$
\mathrm{P}\left[\mathrm{X}_{\mathrm{n}+1} \leq \mathrm{x} \mid \mathrm{V}_{\mathrm{n}}=\mathrm{y}\right]=\frac{\mathrm{F}(\mathrm{x}+\mathrm{y})-\mathrm{F}(\mathrm{y})}{1-\mathrm{F}(\mathrm{y})}
$$


If the degree of the $n$th repair is $a_{n}$, then $V_{n}=V_{n-1}+a_{n} X_{n}$ models the virtual age process. This effectively reduces the aging that took place from $X_{n}$ to $a_{n} X_{n}$. Kijima and other authors refer to this as the Type 1 model. The remainder of the discussion relies on the assumption that all of the $a_{n}$ are identical. Therefore

$$
\mathrm{V}_{\mathrm{n}}=\mathrm{aS}_{\mathrm{n}} \text {, where } \mathrm{S}_{\mathrm{n}}=\sum_{\mathrm{i}=1}^{\mathrm{n}} \mathrm{X}_{\mathrm{i}}
$$

is the expression for the chronological age of the component. A cost model is developed to determine the optimal block replacement interval according to the long run expected cost.

Kijima [1989] proposes a second model for the virtual age process and discusses some properties of models that relax the assumption that all $\mathrm{a}_{\mathrm{n}}$ are identical. The second virtual age process, $V_{n}=a_{n}\left(V_{n-1}+X_{n}\right)$, allows maintenance actions to remove all accumulated age rather than just the aging that took place in the most recent interval between repairs. A simple relationship between the virtual age and chronological age no longer exists in the second model. The exceptions to this statement are if $a_{n}=0, a_{n}=1$, or all $\mathrm{a}_{\mathrm{n}}=\mathrm{a}$ for $0<\mathrm{a}<1$. Therefore, various properties of the chronological age process, $\mathrm{S}$, are investigated. In special cases, bounds and numerical calculations are performed regarding the expected value of $S_{n}$. Numerical results indicate that a large disparity exists between the minimal repair assumption and the general repair assumption in many situations. Kijima and other authors refer to this as the Type II model. A supplemental discussion of various subtle properties associated with the Type I and Type II models is contained in Finkelstein [1993b].

A more flexible model is discussed in Baxter, Kijima, and Tortorella [1996] where the effective age, Y, follows a general distribution, $G(y)$. The effective age is defined as the difference between the degree of effort required to perform a perfect repair and the degree of effort actually expended, transformed into units of operating time. Various properties of the marginal distribution on remaining life length, $\overline{\mathrm{B}}(\mathrm{t})$, are explored where: 


$$
\begin{aligned}
& \bar{B}(t)=\int_{0}^{\infty} \bar{F}(t \mid y) d G(y), \text { for } t \geq 0 \text {, and } \\
& \bar{F}(t \mid y)=\frac{\bar{F}(t+y)}{\bar{F}(y)}
\end{aligned}
$$

Properties of the time between failures are explored under the assumption that the distribution of the effective age is dependent upon the chronological age of the component. Justification for this dependence is given under various scenarios such as improved repairman skill due to experience, improved repair procedures, and improvement or deterioration in available parts and tools. Expressions for the marginal distribution of the time between failures and the generalized renewal function are constructed and properties are discussed.

Dagpunar [1997] considers the case in which the virtual age after the $\mathrm{I}^{\text {th }}$ repair can be expressed as $\mathrm{V}_{\mathrm{i}}=\phi\left(\mathrm{V}_{\mathrm{i}-1}+\mathrm{Y}_{\mathrm{i}}\right)$, where $\mathrm{Y}_{\mathrm{i}}$ is the time between the (I-1) $)^{\text {th }}$ and $\mathrm{I}^{\text {th }}$ repair and $\phi(w)$ is an arbitrary scaling function that models the effects of repair. It is assumed that $0 \leq \phi(\mathrm{w}) \leq \mathrm{w}$ to exclude the possibility of increased wear due to damage during repair. This model is more flexible than the Type II model proposed by Kijima [1989], which models the effects of repair with a series of scalars, $a_{n}$. Integral expressions for the repair density and the joint density of repairs with respect to chronological and virtual age are constructed. A modified policy in which minimal repairs are performed until the component reaches a certain age is explored and the integral expressions for the corresponding repair densities are constructed. Numerical results are given for the repair density over time considering the special case where $\phi(w)=\theta \mathrm{w}$, which is Kijima's Type II model with a constant degree of repair. The restrictive nature of Kijima's Type I model is given as justification for the use of Kijima's Type II model in practice.

Similar results can be found in Dagpunar [1998]. In addition, expressions are developed for the asymptotic value of the expected value of the virtual age and time between repairs. Numerical results based on a cost model are given for the special case of Kijima's Type II model under a block replacement policy. Makis and Jardine [1993] assume a cost structure such that $C_{1}(n, x)$ is the cost of the $n^{\text {th }}$ repair if the age of the 
component is $\mathrm{x}$ and $\mathrm{C}_{0}$ is the cost of a replacement. Under Kijima's Type II policy and the preceding cost structure, it is proved that the optimal replacement policy is as follows: replace at the $\mathrm{n}^{\text {th }}$ failure if it occurs after the time at which the cost of repair exceeds the expected operational cost of the next interval. Comparison between the optimal policy, a block replacement policy, and the assumption of minimal repair are made. The disparity between the minimal repair model and the optimal policy for the general repair model is larger than the disparity found in Kijima [1989]. Results indicate that the minimal repair assumption may only be adequate when the degree of repair is generally poor.

\subsubsection{Scale Factor Model}

Finkelstein [1993a] defines a particular deteriorating renewal process (PDRP) such that $\mathrm{F}_{\mathrm{i}}(\mathrm{x})=\mathrm{F}\left(\mathrm{a}_{\mathrm{i}} \mathrm{x}\right)$, where $1=\mathrm{a}_{1} \leq \mathrm{a}_{2} \leq \mathrm{a}_{3} \leq \ldots \leq \mathrm{a}_{\mathrm{n}}$ and $\mathrm{F}_{\mathrm{i}}(\mathrm{x})$ is the distribution function of the ith time between failures. The only restriction in this model is that successive operating times are stochastically shorter. No indication of a functional relationship or pattern for the series $a_{i}$ is given and all further discussion concerns the special case where $\mathrm{a}_{\mathrm{i}}$ is identical for all $\mathrm{i}$. This model is also discussed in section 2.4.3.

\subsubsection{Additive Age Model}

An improvement factor model simply adds or subtracts a certain amount of age from the accumulated age of the component to model the effects of a repair action. Stadje and Zuckerman [1991] treat the degree of repair as a decision variable that is between the minimal repair and perfect repair cases. A cost model is developed to determine the optimal degree of repair at failure and a decision theory approach is used to identify the optimal type of strategy.

Rangan and Sarada [1993] use a model that incorporates both the virtual age and improvement factor concepts within the framework of a shock model. The shocks arrive according to a NHPP and after each repair activity the accumulated damage is reduced by a constant amount, b. Replacements are made according to a block replacement policy. 
Under these conditions, the virtual age after the $\mathrm{n}^{\text {th }}$ repair can be expressed as $V_{n}=\operatorname{Min}\left\{V_{n-1}+X_{n}-b, 0\right\}$, where $X_{n}$ is the $n^{\text {th }}$ operation time. A cost model is

developed to determine the optimal replacement interval. The authors suggest the general repair case where the degree of repair is a sequence of iid random variables, $\left\{B_{n}\right\}$. This would change the virtual age process to $V_{n}=\operatorname{Min}\left\{V_{n-1}+X_{n}-B_{n}, 0\right\}$. However, all other discussion centers around the assumption that $\mathrm{B}_{\mathrm{n}}=\mathrm{b}$.

The second model proposed in Wang and Pham [1996a] uses the additive age concept to model imperfect PM under a delayed block replacement policy. Each imperfect PM reduces the age of the component by $\mathrm{x}$ units of time. Before $\mathrm{k}$ failures occur, the deterioration of the component is described by the $(\alpha, \beta)$ model below. After $\mathrm{k}$ failures occur, imperfect block replacement is used with periodic replacement. This model is discussed in more detail in the imperfect PM section.

\subsubsection{4 ( $\alpha, \beta)$ Model}

The quasi-renewal process defined in Wang and Pham [1996b, 1997] is an example of an $(\alpha, \beta)$ model. The name indicates that the distribution on the time between failures is reduced by a factor $\alpha$ after each repair, and that the distribution on the repair time is increased each time by a factor $\beta$. The model proposed by Lam [1988] is similar in nature. However, both of these models require a strict relationship between the current and proceeding times between failures. If this relationship is relaxed, this could provide the framework for a general degree of repair after each failure, rather than a predetermined degree of repair after each failure. A more detailed discussion of these two models can be found in section 2.4.3. The same concept is used to a lesser degree in the models contained in Wang and Pham [1996a], which are discussed in more detail in the imperfect PM section. 


\subsubsection{Improvement Factor Model}

Malik [1979] introduced the concept of the improvement factor model. The improvement factor refers to the degree to which PM actions reduce the failure rate of a component. This model is unique, since most of the models in the literature focus on chronological time, operational age, or virtual age to measure the deterioration of a component. A common assumption is that the failure rate is strictly increasing with time. This provides a one-to-one correspondence between any time scale and the failure rate between maintenance actions. Lie and Chun [1986] use the improvement factor approach to model the effect of an imperfect PM action within a more complex maintenance environment than is considered by Malik. The improvement factor in this model is treated as a decision variable and is chosen using a cost model. The improvement factor renders the failure rate somewhere between unchanged and that of a new component. In addition, the optimal number of imperfect PM actions before replacement is obtained. Although the improvement factor model has been used in the context of PM, it has obvious potential for use in general repair models.

\subsection{Inspection Models}

An age replacement policy can only be implemented in an environment where it is possible to monitor the operational age of a component. Many models are based on the assumption that the age and status of a component are monitored continuously and accurately. However, this assumption is often violated in practice. For instance, many safety systems do not show evidence of failure or degradation and require periodic inspection. Most inspection models are based on the assumption that the state of a component is only available upon inspection and that inspections are perfect. ValdezFlores and Feldman [1989] provide a review of inspection models.

Luss [1976] considers an inspection model in which the state of the component is modeled by a finite number of levels of deterioration. The time it takes to deteriorate one level is assumed to follow an exponential distribution. The cost of replacement after 
failure is assumed higher than if the component is replaced at any state prior to failure. A control limit policy is one in which the component is replaced upon detection of a failure or if the accumulated damage has exceeded a threshold upon inspection. This type of policy is used to determine the optimal time until the next inspection, given the level of deterioration at the present inspection.

Zuckerman [1980] constructs a cost model to determine the optimal inspection interval and replacement rule for a component that ages according to a Poisson shock process. The failure probability is a function of the cumulative damage and the magnitude of the most recent shock. In addition, Zuckerman gives a review of various types of inspection models. Abdel-Hameed [1995] summarizes his previous work with inspection models under the assumption of a Markov deterioration process and constant inspection intervals. Properties of the optimal inspection policy are discussed under certain assumptions on the structure of the cost model.

Wortman, Klutke, and Ayhan [1994] evaluate two inspection policies for non-selfannouncing failures that occur as the result of a Poisson shock process. Non-selfannouncing failures commonly occur within security systems and alarm systems, where test or inspection is the only way to detect a failure. An availability model is developed for each policy and availability results are used as a performance measure. Under both of these policies, inspections only reveal whether or not the component is operational or failed. If the component is failed, it is repaired to an "as good as new" condition in negligible time. The only source of downtime is the interval between component failure and the next inspection, since negligible repair times are assumed. The first inspection policy requires that inspections take place randomly according to a stationary renewal process with mean rate $\lambda$. An expression for the time-stationary availability is developed using:

$$
\text { Availability }=\frac{\text { Mean Up Time }}{\text { Mean Up Time }+ \text { Mean Down Time }} .
$$

The second inspection policy requires that inspections be made at regularly spaced intervals of length $1 / \lambda$. The same definition of availability is used and it is shown that the 
deterministic inspection policy provides superior availability performance when compared to the first inspection policy with an identical mean rate.

Vaurio [1999] develops an inspection model under an age replacement policy. Inspections are performed at regular intervals, T. Failures are repaired upon detection and the component is renewed by repair. After $M$ test intervals without failure, the component is renewed through replacement or repair. Constrained optimization is used to find optimal values for $\mathrm{T}$ and $\mathrm{M}$ that minimize cost without violating a restriction on the minimum average availability. 


\section{Chapter 3 - Methodology}

\subsection{Objective}

This research effort is based upon the quasi-renewal process proposed by Lam [1988] and Wang and Pham [1996b, 1997]. Specifically, the quasi-renewal framework is used to develop a continuous availability function over time. The quasi-renewal process is an appealing alternative to the most widely studied imperfect repair model, the $(p, q)$ model proposed by Brown and Proschan [1983].

The $(p, q)$ model requires that subsequent to a failure the system is renewed with probability $p$ and undergoes minimal repair with probability $q=1-p$. Two main results describe the failure intensity and survivor function in terms of the failure intensity and survivor function of a new system, respectively. The failure rate of this model, given no perfect repairs occur in $[0, t)$, is given by $r_{(p, q)}(t)=\operatorname{pr}(t)$, where $r(t)$ is the failure rate of a new system. The survivor function, $\bar{F}_{(p, q)}(t)$, is given by: $\bar{F}_{(p, q)}(t)=[\bar{F}(t)]^{p}$, where $\bar{F}(t)$ is the survivor function of a new system. The remaining results focus on the fact that the class of life distribution is preserved under this model. For instance, if the original life distribution has an increasing failure rate, then the life distribution under this scenario also has an increasing failure rate. Other authors, including Wang and Pham [1996b], have used the $(p, q)$ rule to develop various cost models.

The quasi-renewal model is more appealing than the $(p, q)$ model because it represents the deterioration in post repair equipment status over time. This is much more realistic than fluctuating randomly between the two extreme cases of renewal and minimal repair. Wang and Pham do not exploit the potential for the development of continuous time availability results using the quasi-renewal process. Instead, they assume a relatively complex $\mathrm{PM}$ structure that makes use of the $(p, q)$ model to construct a cost model and optimize the length of the PM interval. The intention of this 
research is to extend the foundation laid by Wang and Pham by developing a probability model of availability, rather than a cost model that incorporates the limiting average availability.

\subsection{Quasi-renewal Process}

Definition 3.1 - Quasi-renewal Process by Wang and Pham [1996b]:

Let $\{N(t), t>0\}$ be a counting process and let $X_{i}$ be the time between the $(i-1)^{\text {th }}$ and the $i^{\text {th }}$ event of the process for $i \geq 1$. Observe the sequence of non-negative random variables $\left\{X_{1}, X_{2}, X_{3}, \ldots\right\}$. The counting process $\{N(t), t>0\}$ is said to be a quasirenewal process with parameter $a$ and the first interarrival time $X_{1}$, if $X_{1}=W_{1}, X_{2}=a W_{2}, X_{3}=a^{2} W_{3}, \ldots$, where the $W_{i}$ are independently and identically distributed and $a>0$ is constant.

The quasi-renewal process describes the scenario where successive intervals of operation, $\left\{X_{1}, X_{2}, X_{3}, \ldots\right\}$, are modeled stochastically as a fraction of the immediately preceding operation interval. The implication of this model is that the distribution function of the $i^{\text {th }}$ interval becomes scaled by a factor, $a^{i-1}$, but retains the same shape. In other words, the scale parameter of the distribution is modified by a factor of $a^{i-1}$, but the distribution family remains the same. A graphical representation of this effect is shown in Figure 3.1. With regard to the density function for the duration of successive operation intervals, the probability mass is compressed toward the y-axis when $a<1$. This increases the likelihood of a shorter operational interval for subsequent intervals and is consistent with the deterioration of a system over time. A graphical representation of this effect is shown in Figure 3.2. In the case $a>1$ the probability mass in the density function is spread away from the y-axis and is consistent with a reliability growth model. When $a=1$ all of the interarrival times are identically distributed and the quasi-renewal process degenerates to an ordinary renewal process. This is the mechanism by which system behavior is described for the purposes of this research. It is important to note that 
this framework can be used to describe some systems that cannot adequately be described using the minimal repair or perfect repair models.

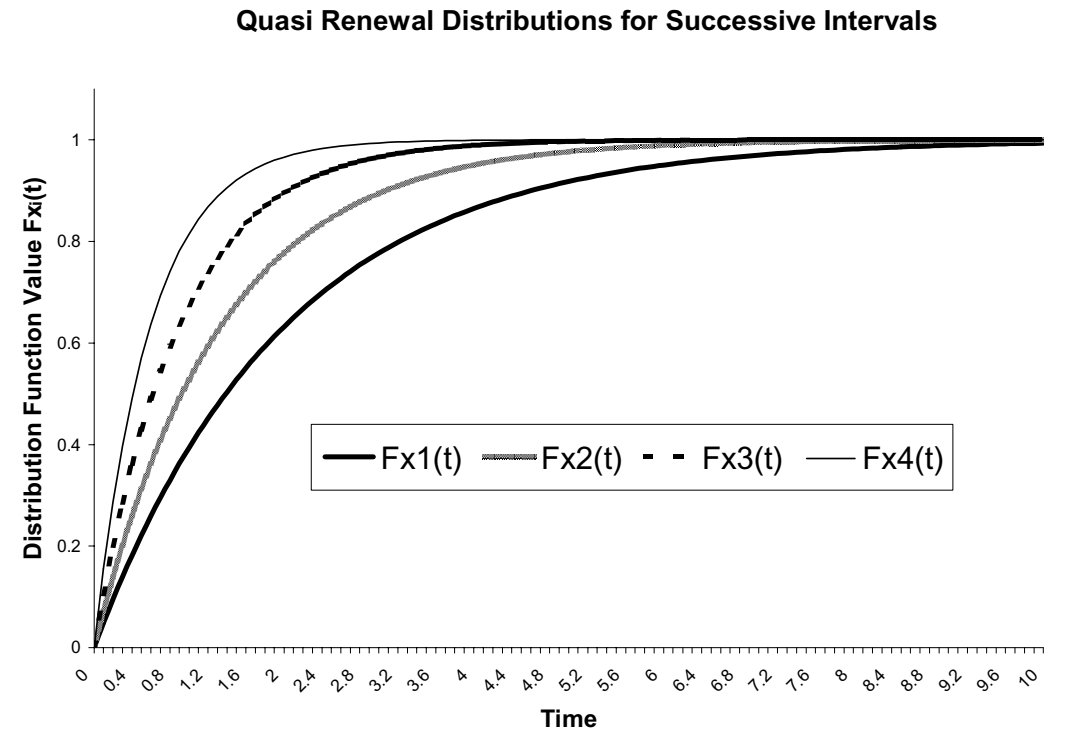

Figure 3.1 Graph of $F_{X_{1}}(t), F_{X_{2}}(t), F_{X_{3}}(t)$, and $F_{X_{4}}(t)$ for a quasi-renewal process

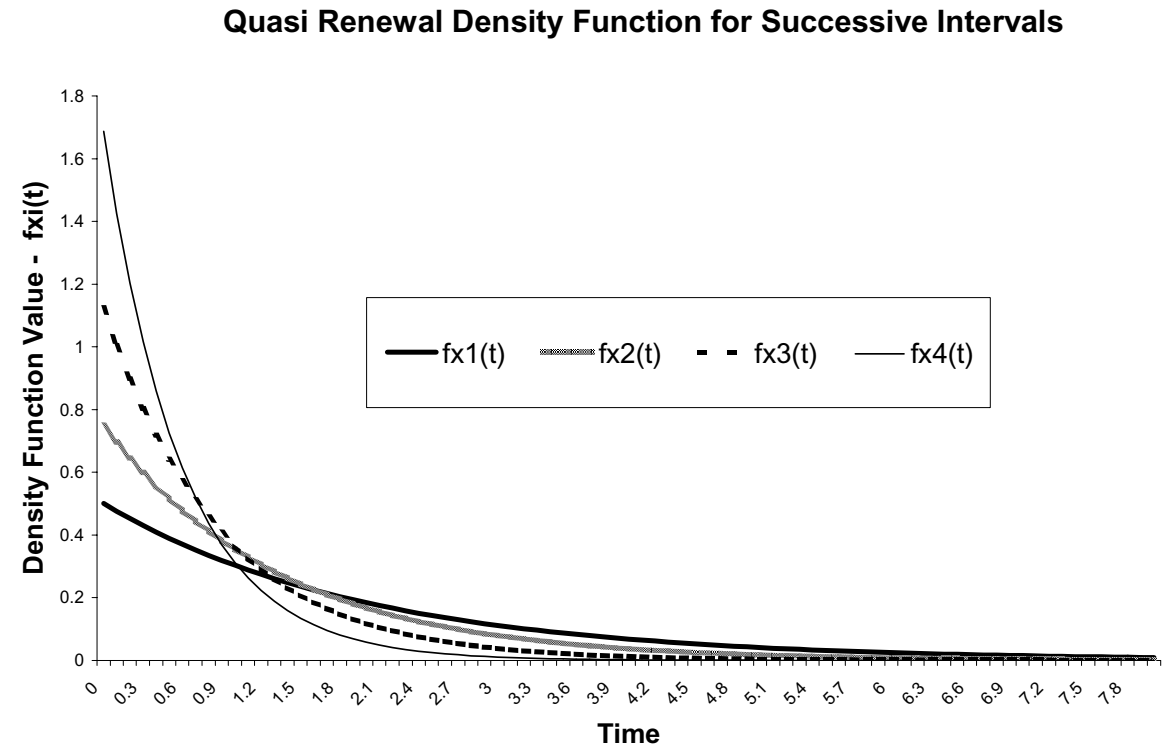

Figure 3.2 Graph of $f_{X_{1}}(t), f_{X_{2}}(t), f_{X_{3}}(t)$, and $f_{X_{4}}(t)$ for a quasi-renewal process 
Let the PDF, CDF, and failure rate of a new system, or equivalently the first operation interval, be $f_{X_{1}}(t), F_{X_{1}}(t)$, and $r_{X_{1}}(t)$ respectively. The PDF, CDF, and failure rate of subsequent lifetimes in a quasi-renewal process can be expressed as follows:

$$
\begin{aligned}
& F_{X_{i}}(t)=F_{X_{1}}\left(\frac{1}{a^{i-1}} t\right), \\
& f_{X_{i}}(t)=\frac{1}{a^{i-1}} f_{X_{1}}\left(\frac{1}{a^{i-1}} t\right), \text { and } \\
& r_{X_{i}}(t)=\frac{1}{a^{i-1}} r_{X_{1}}\left(\frac{1}{a^{i-1}} t\right) .
\end{aligned}
$$

The results in 3.2 and 3.3 can be obtained from the following change of variables theorem for functions of random variables found in Hoel, Port, and Stone [1971]:

Let $\varphi$ be a differentiable strictly increasing or decreasing function on an interval $I$, and let $\varphi(I)$ denote the range of $\varphi$ and $\varphi^{-1}$ the inverse function to $\varphi$. Let $X$ be a continuous random variable having density function $f$ such that $f(x)=0$ for $x \notin I$.

Then $Y=\varphi(X)$ has density $g$ given by $g(y)=0$ for $y \notin \varphi(I)$ and

$$
g(y)=f\left(\varphi^{-1}(y)\right)\left|\frac{d}{d y} \varphi^{-1}(y)\right|, \text { for } y \in \varphi(I) .
$$

The density function of the first interval of operation, $f_{X_{1}}(t)$, is known and the relationship $X_{j}=a^{j-1} X_{1}$ is characteristic of the quasi-renewal process. Therefore, the inverse function is $X_{1}=\frac{1}{a^{j-1}} X_{j}$ and the derivative of the inverse function is $\frac{1}{a^{j-1}}$. Substituting into equation 3.4 provides the result obtained in equation 3.2.

\subsection{Notation}

The system under consideration operates for a period and then is failed for the duration of repair. This continues for the useful life of the system, or until a point at which the system undergoes some type of preventive maintenance. This behavior is shown graphically in Figure 3.3 along with some of the basic notation. 


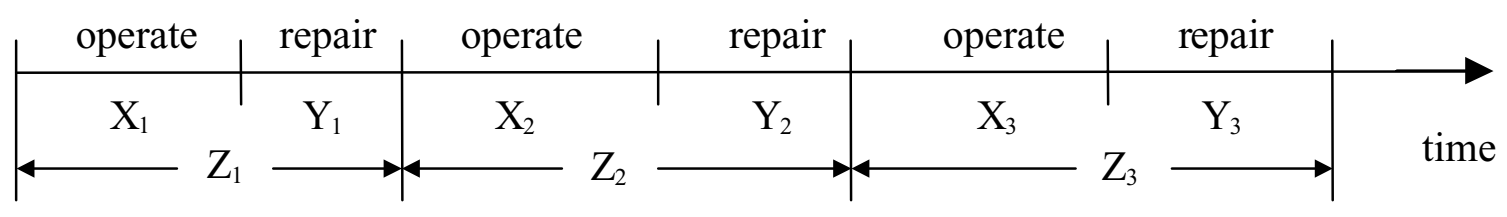

Figure 3.3 Notional System Behavior

Let $X_{i}$ be the random variable that denotes the length of the $i^{\text {th }}$ interval of operation. The PDF, CDF, and failure rate equations for $X_{i}$ are given in 3.1-3.3. It is also useful to define a variable that captures the total operating time of a given number of individual operating times. Let $\Omega_{j}$ be the sum of the first $j$ operating intervals such that $\Omega_{j}=\sum_{i=1}^{j} X_{i}$, and let $F_{\Omega_{j}}(t)$ be the distribution function of the sum of the first $j$ operating intervals such that:

$$
F_{\Omega_{j}}(t)=\operatorname{Pr}\left[\Omega_{j} \leq t\right]=\operatorname{Pr}\left[X_{1}+X_{2}+\ldots+X_{j} \leq t\right]
$$

In order to obtain availability results for the system it is necessary to assume a structure for the non-negligible repair times. Assume the repair times also behave according to a quasi-renewal process with parameter $b \geq 1$. This represents an increase in difficulty and duration for repair as the system ages. Let $Y_{i}$ be the random variable that denotes the length of the $i^{\text {th }}$ repair interval. The PDF and CDF of the repair times can be expressed as follows:

$$
\begin{aligned}
& g_{Y_{i}}(t)=\frac{1}{b^{i-1}} g_{Y_{1}}\left(\frac{1}{b^{i-1}} t\right) \text { and } \\
& G_{Y_{i}}(t)=G_{Y_{1}}\left(\frac{1}{b^{i-1}} t\right) .
\end{aligned}
$$

It is also useful to define a variable that captures the total operating time of a given number of individual repair times. Let $\Psi_{j}$ be the sum of the first $j$ repair intervals such that $\Psi_{j}=\sum_{i=1}^{j} Y_{i}$, and let $G_{\Psi_{j}}(t)$ be the distribution function of the sum of the first $j$ repair intervals such that: 


$$
G_{\Psi_{j}}(t)=\operatorname{Pr}\left[\Psi_{j} \leq t\right]=\operatorname{Pr}\left[Y_{1}+Y_{2}+\ldots+Y_{j} \leq t\right]
$$

To capture the behavior of cycles that contain one operation interval and one repair interval it is useful to define $Z_{i}=X_{i}+Y_{i}$ as the length of the $i^{\text {th }}$ such cycle. The density function of $Z_{i}$ is found using the convolution of the density functions of the $i^{\text {th }}$ operation interval and the $i^{\text {th }}$ repair interval:

$$
\begin{aligned}
h_{Z_{i}}(t) & =\int_{0}^{t} f_{X_{i}}(t-u) g_{Y_{i}}(u) d u, \text { therefore } \\
H_{Z_{i}}(t) & =\int_{0}^{t} F_{X_{i}}(t-u) g_{Y_{i}}(u) d u
\end{aligned}
$$

The total elapsed time of the first $j$ cycles is represented by $\Theta_{j}$, such that $\Theta_{j}=\sum_{i=1}^{j} Z_{i}$. The distribution on the time of the end of the $j^{\text {th }}$ cycle is represented by $H_{\Theta_{j}}(t)$ and can be expressed as follows:

$$
\begin{aligned}
H_{\Theta_{j}}(t) & =\operatorname{Pr}\left[\Theta_{j} \leq t\right] \\
& =\operatorname{Pr}\left[Z_{1}+Z_{2}+\ldots+Z_{j} \leq t\right] \\
& =\operatorname{Pr}\left[X_{1}+Y_{1}+X_{2}+Y_{2}+\ldots+X_{j}+Y_{j} \leq t\right]
\end{aligned}
$$

\subsection{Laplace Transforms}

When working with expressions involving the sums of independent random variables it is often easier work with the corresponding Laplace transforms. Useful information regarding the Laplace transform is found in Abramowitz and Stegun [1972] and Spiegel [1965]. Once an expression in the Laplace transform space is determined, it must be inverted to the domain of the original problem. This may be done analytically in some cases, but more difficult inversion problems may require numerical solution methods. If it exists, the Laplace transform of a positive valued function, $f(t)$, is given by:

$$
L\{f(t)\}=f^{*}(s)=\int_{0}^{\infty} e^{-s t} f(t) d t, \text { for } t>0 .
$$

It should be noted that $s$ is the variable, rather than $t$, in the transform space. The notation for the inverse of the Laplace transform is used in the following statement: 


$$
L^{-1}\{L\{f(t)\}\}=L^{-1}\left\{f^{*}(s)\right\}=f(t)
$$

The density function of the sum of independent random variables can be expressed as the inverse of the product of the Laplace transforms of the density functions in the transform space. This eliminates the necessity to use the integral expression for the convolution formula. Therefore, equation 3.9 can be rewritten:

$$
h_{Z_{i}}(t)=\int_{0}^{t} f_{X_{i}}(t-u) g_{Y_{i}}(u) d u=L^{-1}\left\{f_{X_{i}}^{*}(s) g_{Y_{i}}^{*}(s)\right\}
$$

Two important transform identities allow some of the above equations to be expressed in a more compact and potentially more tractable form.

$$
\begin{aligned}
& L\{F(t)\}=F^{*}(s)=\frac{1}{s} f^{*}(s) \text { and } \\
& L\left\{\frac{1}{a} f\left(\frac{1}{a} t\right)\right\}=f^{*}(a s) \text { for a constant } a>0 .
\end{aligned}
$$

Using the above identities, some of the previous equations can be expressed as follows:

$$
\begin{aligned}
F_{\Omega_{j}}(t) & =\operatorname{Pr}\left[\Omega_{j} \leq t\right]=\operatorname{Pr}\left[X_{1}+X_{2}+\ldots+X_{j} \leq t\right] \\
& =L^{-1}\left\{F_{\Omega_{j}}^{*}(s)\right\}=L^{-1}\left\{\frac{1}{s} f_{\Omega_{j}}^{*}(s)\right\} \\
& =L^{-1}\left\{\frac{1}{s} f_{X_{1}}^{*}(s) f_{X_{2}}^{*}(s) \ldots f_{X_{j}}^{*}(s)\right\} \\
& =L^{-1}\left\{\frac{1}{s} f_{X_{1}}^{*}(s) f_{X_{1}}^{*}(a s) \ldots f_{X_{1}}^{*}\left(a^{j-1} s\right)\right\} \\
G_{\Psi_{j}}(t) & =\operatorname{Pr}\left[\Psi_{j} \leq t\right]=\operatorname{Pr}\left[Y_{1}+Y_{2}+\ldots+Y_{j} \leq t\right] \\
& =L^{-1}\left\{\frac{1}{s} g_{Y_{1}}^{*}(s) g_{Y_{2}}^{*}(s) \ldots g_{Y_{j}}^{*}(s)\right\} \\
& =L^{-1}\left\{\frac{1}{s} g_{Y_{1}}^{*}(s) g_{Y_{1}}^{*}(b s) \ldots g_{Y_{1}}^{*}\left(b^{j-1} s\right)\right\}
\end{aligned}
$$




$$
\begin{aligned}
H_{\Theta_{j}}(t) & =\operatorname{Pr}\left[\Theta_{j} \leq t\right]=\operatorname{Pr}\left[Z_{1}+Z_{2}+\ldots+Z_{j} \leq t\right] \\
& =\operatorname{Pr}\left[X_{1}+Y_{1}+\ldots+X_{j}+Y_{j} \leq t\right] \\
& =L^{-1}\left\{\frac{1}{s} h_{Z_{1}}^{*}(s) h_{Z_{2}}^{*}(s) \ldots h_{Z_{j}}^{*}(s)\right\} \\
& =L^{-1}\left\{\frac{1}{s} f_{X_{1}}^{*}(s) f_{X_{2}}^{*}(s) \ldots f_{X_{j}}^{*}(s) g_{Y_{1}}^{*}(s) g_{Y_{2}}^{*}(s) \ldots g_{Y_{j}}^{*}(s)\right\} \\
& =L^{-1}\left\{\frac{1}{s} f_{X_{1}}^{*}(s) f_{X_{1}}^{*}(a s) \ldots f_{X_{1}}^{*}\left(a^{j-1} s\right) g_{Y_{1}}^{*}(s) g_{Y_{1}}^{*}(b s) \ldots g_{Y_{1}}^{*}\left(b^{j-1} s\right)\right\}
\end{aligned}
$$

The first two relationships, 3.17 and 3.18, allow the distribution functions on the sum of $j$ operating intervals or repair intervals to be expressed in terms of the distribution of the first operating interval or the first repair interval, respectively. The distribution function on the cycle times is not as compact since the distinct values of $a$ and $b$ make further simplification difficult. However, it is possible exploit the straightforward relationship that $h_{\Theta_{j}}^{*}(s)=f_{\Omega_{j}}^{*}(s) g_{\Psi_{j}}^{*}(s)$ as shown in 3.19.

\subsection{Model Development}

\subsubsection{The Quasi-renewal Function, $Q(t)$}

Important in the study of renewal processes is the form of the renewal function and the renewal density. The development of similar expressions for the quasi-renewal process is the first step toward the goal of a point availability expression. Unlike a basic renewal process, the lifetimes of the quasi-renewal process are not identically distributed. However, the relationship between the $j^{\text {th }}$ interval of operation and the first interval of operation is straightforward, as stated in 3.1 and 3.2. Let $Q(t)$ denote the parallel to the renewal function for the quasi-renewal process, that is the function for the expected number of restarts. Note that repair times are considered negligible in this development. 


$$
\begin{aligned}
Q_{F}(t) & =E[N(t)]=\sum_{n=0}^{\infty} n \operatorname{Pr}[N(t)=n]=\sum_{n=1}^{\infty} n(\operatorname{Pr}[N(t) \geq n]-\operatorname{Pr}[N(t) \geq n+1]) \\
& =\sum_{n=1}^{\infty} n\left(\operatorname{Pr}\left[X_{1}+X_{2}+\ldots+X_{n} \leq t\right]-\operatorname{Pr}\left[X_{1}+X_{2}+\ldots+X_{n+1} \leq t\right]\right) \\
& =\sum_{n=1}^{\infty} n\left(F_{\Omega_{n}}(t)-F_{\Omega_{n+1}}(t)\right) \\
& =\sum_{n=1}^{\infty} F_{\Omega_{n}}(t)
\end{aligned}
$$

It is useful to work in the transform space from this point.

$$
\begin{aligned}
Q^{*}(s) & =L\{Q(t)\}=L\left\{\sum_{n=1}^{\infty} F_{\Omega_{n}}(t)\right\} \\
& =\sum_{n=1}^{\infty} \frac{1}{s} f_{X_{1}}^{*}(s) f_{X_{2}}^{*}(s) \ldots f_{X_{n}}^{*}(s) \\
& =\frac{1}{s}\left[f_{X_{1}}^{*}(s)+\sum_{n=2}^{\infty} f_{X_{1}}^{*}(s) f_{X_{2}}^{*}(s) \ldots f_{X_{n}}^{*}(s)\right] \\
& =\frac{1}{s}\left[f_{X_{1}}^{*}(s)+f_{X_{1}}^{*}(s) \sum_{n=2}^{\infty} f_{X_{2}}^{*}(s) f_{X_{3}}^{*}(s) \ldots f_{X_{n}}^{*}(s)\right] \\
& =\frac{1}{s}\left[f_{X_{1}}^{*}(s)+f_{X_{1}}^{*}(s) \sum_{n-1=1}^{\infty} f_{X_{2}}^{*}(s) f_{X_{3}}^{*}(s) \ldots f_{X_{n}}^{*}(s)\right]
\end{aligned}
$$

The transform identity stated in 3.16 can be used to express the transform of the $j^{\text {th }}$ operation interval density function in terms of the transform of the first operation interval density function:

$$
\begin{aligned}
& f_{X_{j}}(t)=\frac{1}{a^{j-1}} f_{X_{1}}\left(\frac{1}{a^{j-1}} t\right), \text { therefore } \\
& f_{X_{j}}^{*}(s)=f_{X_{1}}^{*}\left(a^{j-1} s\right) .
\end{aligned}
$$

Another useful relationship is given by

$$
f_{X_{j}}^{*}(s)=f_{X_{1}}^{*}\left(a^{j-1} s\right)=f_{X_{1}}^{*}\left(a^{j-2} a s\right)=f_{X_{j-1}}^{*}(a s) .
$$


Starting with the result in 3.21 and using the results in 3.22 and 3.23

$$
\begin{aligned}
Q^{*}(s) & =\frac{1}{s}\left[f_{X_{1}}^{*}(s)+f_{X_{1}}^{*}(s) \sum_{n-1=1}^{\infty} f_{X_{2}}^{*}(s) f_{X_{3}}^{*}(s) \ldots f_{X_{n}}^{*}(s)\right] \\
& =\frac{1}{s}\left[f_{X_{1}}^{*}(s)+f_{X_{1}}^{*}(s) \sum_{n-1=1}^{\infty} f_{X_{1}}^{*}(a s) f_{X_{2}}^{*}(a s) \ldots f_{X_{n-1}}^{*}(a s)\right] \\
& =\frac{1}{s}\left[f_{X_{1}}^{*}(s)+f_{X_{1}}^{*}(s) q^{*}(a s)\right] \\
& =F_{X_{1}}^{*}(s)+f_{X_{1}}^{*}(s) Q^{*}(a s) \\
& =\frac{1}{s} f_{X_{1}}^{*}(s)+f_{X_{1}}^{*}(s) Q^{*}(a s) \\
& =F_{X_{1}}^{*}(s)+s F_{X_{1}}^{*}(s) Q^{*}(a s)
\end{aligned}
$$

An expression in the original time domain is found by inverting the expression $Q^{*}(s)=F_{X_{1}}^{*}(s)+f_{X_{1}}^{*}(s) Q^{*}(a s)$ to obtain

$$
\begin{aligned}
& L^{-1}\left\{Q^{*}(s)\right\}=L^{-1}\left\{F_{X_{1}}^{*}(s)+f_{X_{1}}^{*}(s) Q^{*}(a s)\right\} \\
& Q(t)=F_{X_{1}}(t)+\frac{1}{a} f_{X_{1}}(t) \oplus Q\left(\frac{1}{a} t\right),
\end{aligned}
$$

where $\oplus$ is the binary operator that denotes the convolution of two distributions.

After rearranging terms in 3.24 an interesting relationship between the quasi-renewal function and the distribution of the first interval of operation becomes apparent,

$$
f_{X_{1}}^{*}(s)=\frac{Q^{*}(s)}{\frac{1}{s}+Q^{*}(a s)} \text { or equivalently } F_{X_{1}}^{*}(s)=\frac{Q^{*}(s)}{1+s Q^{*}(a s)} .
$$

Because the relationship between $Q^{*}(s)$ and $Q^{*}(a s)$ is not subject to convenient analysis, we cannot write $Q(t)$ as a function of $F(t)$. Nevertheless, it may be possible to use 3.26 to obtain results numerically. This result is not obtained by Wang and Pham and is a key result that may prove useful in future analysis of the quasi-renewal process.

\subsubsection{Point Availability}

Defining an expression for the point availability of the quasi-renewal process is complicated by the distinct lifetime and repair distributions for each lifetime and repair 
interval. Since the behavior of the process is cycle dependent, a simple statement using the quasi-renewal function is not possible. This is because the quasi-renewal function based on cycles including repair is analytically intractable. However, the characteristic relationship between the successive lifetime and repair intervals of the quasi-renewal process may be exploited to provide an appropriate description of its availability behavior.

$$
\begin{aligned}
A(t)= & \bar{F}_{X_{1}}(t)+\int_{0}^{t} \bar{F}_{X_{2}}(t-u) h_{\Theta_{1}}(u) d u+ \\
& \int_{0}^{t} \bar{F}_{X_{3}}(t-u) h_{\Theta_{2}}(u) d u+\ldots+\int_{0}^{t} \bar{F}_{X_{n+1}}(t-u) h_{\Theta_{n}}(u) d u+\ldots,
\end{aligned}
$$

where $h_{\Theta_{j}}(u)=\frac{d}{d u} H_{\Theta_{j}}(u)$. The first term in the availability function reflects the probability that the first period of operation is of length $t$ or greater. The subsequent integral expressions reflect the probability that the $n^{\text {th }}$ failure occurs at time $u$ and the following period of operation is of length $(t-u)$ or greater. A more compact expression of the above availability function is:

$$
A(t)=\bar{F}_{X_{1}}(t)+\sum_{n=1}^{\infty} \int_{0}^{t} \bar{F}_{X_{n+1}}(t-u) h_{\Theta_{n}}(u) d u .
$$

An equivalent expression in the transform space is:

$$
\begin{aligned}
& L\{A(t)\}=L\left\{\bar{F}_{X_{1}}(t)\right\}+\sum_{n=1}^{\infty} L\left\{\int_{0}^{t} \bar{F}_{X_{n+1}}(t-u) h_{\Theta_{n}}(u) d u\right\} \\
& A^{*}(s)=L\left\{1-F_{X_{1}}(t)\right\}+\sum_{n=1}^{\infty} L\left\{\int_{0}^{t} h_{\Theta_{n}}(u) d u\right\}-\sum_{n=1}^{\infty} L\left\{\int_{0}^{t} F_{X_{n+1}}(t-u) h_{\Theta_{n}}(u) d u\right\} \\
& A^{*}(s)=\frac{1}{S}\left(1-f_{X_{1}}^{*}(s)\right)+\sum_{n=1}^{\infty} H_{\Theta_{n}}^{*}(s)-\sum_{n=1}^{\infty} F_{X_{n+1}}^{*}(s) h_{\Theta_{n}}^{*}(s) \\
& A^{*}(s)=\frac{1}{S}\left[\left(1-f_{X_{1}}^{*}(s)\right)+\sum_{n=1}^{\infty} h_{\Theta_{n}}^{*}(s)-\sum_{n=1}^{\infty} f_{X_{n+1}}^{*}(s) h_{\Theta_{n}}^{*}(s)\right] \\
& A^{*}(s)=\frac{1}{S}\left[\left(1-f_{X_{1}}^{*}(s)\right)+\sum_{n=1}^{\infty}\left(1-f_{X_{n+1}}^{*}(s)\right) h_{\Theta_{n}}^{*}(s)\right] .
\end{aligned}
$$

Since the availability expression in 3.29 is derived from 3.28 and reflects the probability that the system is operational at any point in time $t, 3.29$ is referred to as the uptimebased point availability function. 
A second method of deriving an expression for the point availability is discussed below. First, an expression for the special case where repair intervals are independent and identically distributed (iid) is developed. Starting with 3.29

$$
\begin{aligned}
& A^{*}(s)=\frac{1}{s}\left[\left(1-f_{X_{1}}^{*}(s)\right)+\sum_{n=1}^{\infty}\left(1-f_{X_{n+1}}^{*}(s)\right) h_{\Theta_{n}}^{*}(s)\right] \\
& A^{*}(s)=\frac{1}{s}\left[\left(1-f_{X_{1}}^{*}(s)\right)+\sum_{n=1}^{\infty}\left(1-f_{X_{n+1}}^{*}(s)\right) f_{X_{1}}^{*}(s) \ldots f_{X_{n}}^{*}(s) g_{Y_{1}}^{*}(s) \ldots g_{Y_{n}}^{*}(s)\right] \\
& A^{*}(s)=\frac{1}{s}\left[\left(1-f_{X_{1}}^{*}(s)\right)+\sum_{n=1}^{\infty}\left(1-f_{X_{n+1}}^{*}(s)\right)\left(g_{Y_{1}}^{*}(s)\right)^{n} \prod_{i=1}^{n} f_{X_{i}}^{*}(s)\right] .
\end{aligned}
$$

The expression in 3.30 is referred to as the uptime-based point availability function under iid repair.

Another way to develop an expression for the point availability is based on the complement of the event the system is down at any point in time $t$. In the time domain this is expressed as follows

$$
\begin{aligned}
A(t) & =1-\operatorname{Pr}[\text { System Down at time } t] \\
& =1-\left[f_{X_{1}}(u) \oplus \bar{G}_{Y_{1}}(t-u)+f_{Z_{1}+X_{2}}(u) \oplus \bar{G}_{Y_{2}}(t-u)+\ldots\right] .
\end{aligned}
$$

Equation 3.31 reflects the probability that the $n^{\text {th }}$ interval of operation ends at time $u$ and the $n^{\text {th }}$ repair interval is $(t-u)$ time units or greater. Working in the transform space gives the following result

$$
\begin{aligned}
A^{*}(s) & =\frac{1}{s}\left[1-\left(f_{X_{1}}^{*}(s)\left(1-g_{Y_{1}}^{*}(s)\right)+f_{X_{1}}^{*}(s) g_{Y_{1}}^{*}(s) f_{X_{2}}^{*}(s)\left(1-g_{Y_{2}}^{*}(s)\right)+\ldots\right)\right] \\
& =\frac{1}{s}\left[1-\sum_{n=1}^{\infty}\left(1-g_{Y_{n}}^{*}(s)\right) f_{X_{1}}^{*}(s) \ldots f_{X_{n}}^{*}(s) g_{Y_{1}}^{*}(s) \ldots g_{Y_{n-1}}^{*}(s)\right] \\
& =\frac{1}{s}\left[1-\sum_{n=1}^{\infty} f_{X_{1}}^{*}(s) \ldots f_{X_{n}}^{*}(s) g_{Y_{1}}^{*}(s) \ldots g_{Y_{n-1}}^{*}(s)-f_{X_{1}}^{*}(s) \ldots f_{X_{n}}^{*}(s) g_{Y_{1}}^{*}(s) \ldots g_{Y_{n}}^{*}(s)\right] \\
& =\frac{1}{s}\left[1-\sum_{n=1}^{\infty}\left(\frac{1}{g_{Y_{n}}^{*}(s)}-1\right) h_{\Theta_{n}}^{*}(s)\right] .
\end{aligned}
$$

Since the availability expression in 3.32 is derived from 3.31 and reflects the complement of the probability that the system is down at any point in time $t, 3.32$ is referred to as the downtime-based point availability function. 
The iid repair interval case allows the following simplification

$$
\begin{aligned}
A^{*}(s) & =\frac{1}{s}-\frac{1}{s} \sum_{n=1}^{\infty}\left(\frac{1}{g_{Y_{n}}^{*}(s)}-1\right) h_{\Theta_{n}}^{*}(s) \\
& =\frac{1}{s}-\frac{1}{s} \sum_{n=1}^{\infty}\left(\frac{1}{g_{Y_{n}}^{*}(s)}-1\right) f_{X_{1}}^{*}(s) \ldots f_{X_{n}}^{*}(s) g_{Y_{1}}^{*}(s) \ldots g_{Y_{n}}^{*}(s) \\
& =\frac{1}{s}\left[1-\left(\frac{1}{g_{Y_{1}}^{*}(s)}-1\right) \sum_{n=1}^{\infty}\left(g_{Y_{1}}^{*}(s)\right)^{n} \prod_{i=1}^{n} f_{X_{i}}^{*}(s)\right]
\end{aligned}
$$

This expression is referred to as the downtime-based point availability function under iid repair.

It is important to recognize that the downtime-based point availability function and the uptime-based point availability function are exact and equivalent. However, both expressions are stated in terms of infinite sums that must be truncated in order to invert the transform back to the time domain and perform numerical calculations. Therefore, any numerical work performed using these expressions is approximate. The degree of approximation is reduced as the point of truncation, the maximum value of $n$, increases.

\subsubsection{Bounds on the Point Availability}

The uptime-based and downtime-based point availability functions provide lower and upper bounds on the point availability. This follows from the fact that each term of the infinite sum in the exact point availability equations $3.29,3.30,3.32$, and 3.33 corresponds to an event with positive probability in the time domain. Let $c$ be a nonnegative integer that represents the point of truncation for the purposes of inversion and numerical work. The resulting equations for the approximate calculation of the point availability are as follows

$$
\begin{aligned}
& A^{*}(s)=\frac{1}{s}\left[\left(1-f_{X_{1}}^{*}(s)\right)+\sum_{n=1}^{c}\left(1-f_{X_{n+1}}^{*}(s)\right) h_{\Theta_{n}}^{*}(s)\right], \\
& A^{*}(s)=\frac{1}{s}\left[\left(1-f_{X_{1}}^{*}(s)\right)+\sum_{n=1}^{c}\left(1-f_{X_{n+1}}^{*}(s)\right)\left(g_{Y_{1}}^{*}(s)\right)^{n} \prod_{i=1}^{n} f_{X_{i}}^{*}(s)\right],
\end{aligned}
$$




$$
\begin{aligned}
& A^{*}(s)=\frac{1}{s}\left[1-\sum_{n=1}^{c}\left(\frac{1}{g_{Y_{n}}^{*}(s)}-1\right) h_{\Theta_{n}}^{*}(s)\right], \text { and } \\
& A^{*}(s)=\frac{1}{s}\left[1-\left(\frac{1}{g_{Y_{1}}^{*}(s)}-1\right) \sum_{n=1}^{c}\left(g_{Y_{1}}^{*}(s)\right)^{n} \prod_{i=1}^{n} f_{X_{i}}^{*}(s)\right] .
\end{aligned}
$$

Equations 3.34 and 3.35 are the truncated uptime-based point availability functions and are increasing in $c$, forming a lower bound on the point availability that becomes tighter as $c$ increases. Similarly, equations 3.36 and 3.37 are the truncated downtime-based point availability functions and are decreasing in $c$, forming an upper bound on the point availability that becomes tighter as $c$ increases.

The bounds described above are also used to provide the maximum error for the calculation of the point availability at any point in time. Subtracting the value of the uptime-based availability function from the downtime-based availability function at time $t$ provides the maximum error in the calculated point availability at time $t$. Let $\operatorname{Error}(t)$ be the maximum error of the point availability calculation at time $t$ when $c$ is the point of truncation for both the uptime-based and downtime-based point availability functions.

$$
\begin{aligned}
& \operatorname{Error}(t)=L^{-1}\left\{\begin{array}{l}
\frac{1}{s}\left[1-\sum_{n=1}^{c}\left(\frac{1}{g_{Y_{n}}^{*}(s)}-1\right) h_{\Theta_{n}}^{*}(s)\right]- \\
\frac{1}{s}\left[\left(1-f_{X_{1}}^{*}(s)\right)+\sum_{n=1}^{c}\left(1-f_{X_{n+1}}^{*}(s)\right) h_{\Theta_{n}}^{*}(s)\right]
\end{array}\right\} \\
& =L^{-1}\left\{\frac{1}{s}\left[\begin{array}{l}
1-\sum_{n=1}^{c}\left(\frac{1}{g_{Y_{n}}^{*}(s)}-1\right) h_{\Theta_{n}}^{*}(s)-\left(1-f_{X_{1}}^{*}(s)\right) \\
-\sum_{n=1}^{c}\left(1-f_{X_{n+1}}^{*}(s)\right) h_{\Theta_{n}}^{*}(s)
\end{array}\right]\right\} \\
& =L^{-1}\left\{\frac{1}{s}\left[f_{X_{1}}^{*}(s)-\sum_{n=1}^{c}\left(\frac{1}{g_{Y_{n}}^{*}(s)}-1\right) h_{\Theta_{n}}^{*}(s)+\left(1-f_{X_{n+1}}^{*}(s)\right) h_{\Theta_{n}}^{*}(s)\right]\right\} \\
& =L^{-1}\left\{\frac{1}{s}\left[f_{X_{1}}^{*}(s)-\sum_{n=1}^{c}\left(\frac{1}{g_{Y_{n}}^{*}(s)}-f_{X_{n+1}}^{*}(s)\right) h_{\Theta_{n}}^{*}(s)\right]\right\}
\end{aligned}
$$


As expected, the error function is decreasing in $c$. This expression for the maximum error can be used to determine the minimum value of $c$ necessary to achieve any specified degree of accuracy in the calculation of the point availability. This application is discussed in detail in Chapter 4.

The general framework for the probability-based point availability analysis of the quasi-renewal process is provided above. The application of this framework under various assumptions on the distribution of the operation and repair intervals is provided below. Specifically, the operation and repair intervals are assumed to behave according to the exponential, normal, and gamma distributions. The specific models are constructed below and a discussion of the numerical results and behavior of each model is contained in Chapter 4.

\subsection{Quasi-Renewal Model Under Exponential Operating Intervals}

The quasi-renewal function is constructed and is the foundation for the development of the availability expressions under exponentially distributed operating intervals. Uptime-based and downtime-based point availability functions are constructed under the assumption of exponentially distributed quasi-renewal operating intervals for both the quasi-renewal repair case and the iid repair case. Inversion of the exact transform expression is not possible without truncation. Therefore the exact expression in the transform space is provided rather than an approximate expression in the time domain. Let the duration of the first interval of operation be distributed as follows

$$
X_{1} \sim \exp (\lambda) \rightarrow f_{X_{1}}(t)=\lambda e^{-\lambda t} \text { for } t>0 .
$$

The Laplace transform of the density function of the $n^{\text {th }}$ operation interval is

$$
\begin{aligned}
& f_{X_{1}}^{*}(s)=L^{-1}\left\{f_{X_{1}}(t)\right\}=\frac{\lambda}{\lambda+s} \\
& f_{X_{n}}^{*}(s)=f_{X_{1}}^{*}\left(a^{n-1} s\right)=\frac{\lambda}{\lambda+a^{n-1} s} .
\end{aligned}
$$

For this discussion it is assumed that the lengths of the operation intervals are stochastically decreasing and therefore $0<a<1$. Similarly it is assumed that the lengths 
of operating intervals are stochastically increasing and therefore $b<1$. It is trivial to modify the expressions obtained under these two assumptions to reflect the opposite behavior. Let the duration of the first repair interval be distributed as follows

$$
Y_{1} \sim \exp (\mu) \rightarrow g_{Y_{1}}(t)=\mu e^{-\mu t} \text { for } t>0 .
$$

The Laplace transform of the density function of the $n^{\text {th }}$ repair interval is

$$
\begin{aligned}
& g_{Y_{1}}^{*}(s)=L^{-1}\left\{g_{Y_{1}}(t)\right\}=\frac{\mu}{\mu+s} \\
& g_{Y_{n}}^{*}(s)=g_{Y_{1}}^{*}\left(b^{n-1} s\right)=\frac{\mu}{\mu+b^{n-1} s} .
\end{aligned}
$$

\subsubsection{Exponential Quasi-Renewal Function}

It is established that the quasi-renewal function is given by

$$
Q^{*}(s)=\sum_{n=1}^{\infty} F_{\Omega_{n}}^{*}(s)=\frac{1}{s} \sum_{n=1}^{\infty} \prod_{i=1}^{n} f_{X_{i}}^{*}(s)=\frac{1}{s} \sum_{n=1}^{\infty} \prod_{i=1}^{n} f_{X_{1}}^{*}\left(a^{i-1} s\right) .
$$

Therefore, the quasi-renewal function for exponentially distributed operating intervals is given by

$$
Q^{*}(s)=\frac{1}{s} \sum_{n=1}^{\infty} \prod_{i=1}^{n} f_{X_{1}}^{*}\left(a^{i-1} s\right)=\frac{1}{s} \sum_{n=1}^{\infty} \prod_{i=1}^{n} \frac{\lambda}{\lambda+a^{i-1} s} .
$$

\subsubsection{Uptime-Based Point Availability Function with Exponential Repair}

The uptime-based point availability function with exponential quasi-renewal operating intervals and exponential quasi-renewal repair intervals is given by

$$
\begin{aligned}
A^{*}(s) & =\frac{1}{s}\left[\left(1-f_{X_{1}}^{*}(s)\right)+\sum_{n=1}^{\infty}\left(1-f_{X_{n+1}}^{*}(s)\right) h_{\Theta_{n}}^{*}(s)\right] \\
& =\frac{1}{s}\left[\left(1-\frac{\lambda}{\lambda+s}\right)+\sum_{n=1}^{\infty}\left(1-\frac{\lambda}{\lambda+a^{n} s}\right) \prod_{i=1}^{n} \frac{\mu \lambda}{\left(\lambda+a^{i-1} s\right)\left(\mu+b^{i-1} s\right)}\right] \\
& =\frac{1}{s}\left[\left(\frac{s}{\lambda+s}\right)+\sum_{n=1}^{\infty}\left(\frac{a^{n} s}{\lambda+a^{n} s} \prod_{i=1}^{n} \frac{\mu \lambda}{\left(\lambda+a^{i-1} s\right)\left(\mu+b^{i-1} s\right)}\right)\right] \\
& =\left(\frac{1}{\lambda+s}\right)+\sum_{n=1}^{\infty}\left(\frac{(a \mu \lambda)^{n}}{\lambda+a^{n} s} \prod_{i=1}^{n} \frac{1}{\left(\lambda+a^{i-1} s\right)\left(\mu+b^{i-1} s\right)}\right)
\end{aligned}
$$


The uptime-based point availability function under iid repair with exponential quasirenewal operating intervals and exponential iid repair intervals is given by

$$
\begin{aligned}
A^{*}(s) & =\frac{1}{s}\left[\left(1-f_{X_{1}}^{*}(s)\right)+\sum_{n=1}^{\infty}\left(1-f_{X_{n+1}}^{*}(s)\right)\left(g_{Y_{1}}^{*}(s)\right)^{n} \prod_{i=1}^{n} f_{X_{i}}^{*}(s)\right] \\
& =\frac{1}{s}\left[\left(1-\frac{\lambda}{\lambda+s}\right)+\sum_{n=1}^{\infty}\left(1-\frac{\lambda}{\lambda+a^{n} s}\right)\left(\frac{\mu}{\mu+s}\right)^{n} \prod_{i=1}^{n} \frac{\lambda}{\lambda+a^{i-1} s}\right] \\
& =\frac{1}{s}\left[\frac{s}{\lambda+s}+\sum_{n=1}^{\infty} \frac{a^{n} s}{\lambda+a^{n} s}\left(\frac{\mu}{\mu+s}\right)^{n} \prod_{i=1}^{n} \frac{\lambda}{\lambda+a^{i-1} s}\right] \\
& =\frac{1}{\lambda+s}+\sum_{n=1}^{\infty} \frac{a^{n}}{\lambda+a^{n} s}\left(\frac{\mu \lambda}{\mu+s}\right)^{n} \prod_{i=1}^{n} \frac{1}{\lambda+a^{i-1} s}
\end{aligned}
$$

\subsubsection{Downtime-Based Point Availability Function with Exponential Repair}

The downtime-based point availability function with exponential quasi-renewal operating intervals and exponential quasi-renewal repair intervals is given by

$$
\begin{aligned}
A^{*}(s) & =\frac{1}{s}\left[1-\sum_{n=1}^{\infty}\left(\frac{1}{g_{Y_{n}}^{*}(s)}-1\right) h_{\Theta_{n}}^{*}(s)\right] \\
& =\frac{1}{s}\left[1-\sum_{n=1}^{\infty}\left(\frac{\mu+b^{n-1} s}{\mu}-1\right) \prod_{i=1}^{n} \frac{\lambda}{\lambda+a^{i-1} s} \frac{\mu}{\mu+b^{i-1} s}\right] \\
& =\frac{1}{s}-\frac{1}{\mu} \sum_{n=1}^{\infty} b^{n-1}(\mu \lambda)^{n} \prod_{i=1}^{n} \frac{1}{\left(\lambda+a^{i-1} s\right)\left(\mu+b^{i-1} s\right)}
\end{aligned}
$$


The downtime-based point availability function under iid repair with exponential quasi-renewal operating intervals and exponential iid repair intervals is given by

$$
\begin{aligned}
A^{*}(s) & =\frac{1}{s}\left[1-\left(\frac{1}{g_{Y_{1}}^{*}(s)}-1\right) \sum_{n=1}^{\infty}\left(g_{Y_{1}}^{*}(s)\right)^{n} \prod_{i=1}^{n} f_{X_{i}}^{*}(s)\right] \\
& =\frac{1}{s}\left[1-\left(\frac{\mu+s}{\mu}-1\right) \sum_{n=1}^{\infty}\left(\frac{\mu}{\mu+s}\right)^{n} \prod_{i=1}^{n} \frac{\lambda}{\lambda+a^{i-1} s}\right] \\
& =\frac{1}{s}-\frac{1}{\mu} \sum_{n=1}^{\infty}\left(\frac{\mu \lambda}{\mu+s}\right)^{n} \prod_{i=1}^{n} \frac{1}{\lambda+a^{i-1} s}
\end{aligned}
$$

\subsection{Quasi-Renewal Model Under Normal Operating Intervals}

The quasi-renewal function is constructed and is the foundation for the development of the availability expressions under normally distributed operating intervals. Uptimebased and downtime-based point availability functions are constructed under the assumption of normally distributed quasi-renewal operating intervals for both the quasirenewal repair case and the iid repair case. The repair intervals are modeled using both the normal distribution and the exponential distribution. Under the assumption of normally distributed repair intervals the transform of the point availability is easily inverted and expressed as a function of time. Under the assumption of exponentially distributed repair intervals inversion of the exact transform expression is not possible without truncation. Therefore the exact expression in the transform space is provided rather than an approximate expression in the time domain. Let the duration of the first interval of operation be distributed as follows

$$
X_{1} \sim \operatorname{normal}\left(\mu, \sigma^{2}\right) \rightarrow f_{X_{1}}(t)=\frac{1}{\sigma \sqrt{2 \pi}} e^{\frac{-(t-\mu)^{2}}{2 \sigma^{2}}} .
$$

In addition, let $\Phi$ represent the cumulative distribution function of the normal distribution,

$$
\Phi\left(\mu, \sigma^{2}\right)=\int_{-\infty}^{\infty} \frac{1}{\sigma \sqrt{2 \pi}} e^{\frac{-(t-\mu)^{2}}{2 \sigma^{2}}} d t .
$$


For the purpose of this analysis it is assumed that the mean and variance of the normal distribution are such that the probability mass located at $t<0$ is negligible. The Laplace transform of the density function of the $n^{\text {th }}$ operation interval is

$$
\begin{aligned}
& f_{X_{1}}^{*}(s)=L^{-1}\left\{f_{X_{1}}(t)\right\}=e^{\mu s-\frac{1}{2} \sigma^{2} s^{2}} \\
& f_{X_{n}}^{*}(s)=f_{X_{1}}^{*}\left(a^{n-1} s\right)=e^{\mu a^{n-1} s-\frac{1}{2} \sigma^{2}\left(a^{n-1} s\right)^{2}} .
\end{aligned}
$$

\subsubsection{Normal Quasi-Renewal Function}

The quasi-renewal function for normally distributed operating intervals is given by

$$
\begin{aligned}
Q^{*}(s) & =\frac{1}{s} \sum_{n=1}^{\infty} \prod_{i=1}^{n} f_{X_{1}}^{*}\left(a^{i-1} s\right) \\
& =\frac{1}{s} \sum_{n=1}^{\infty} \prod_{i=1}^{n} e^{\mu a^{i-1} s-\frac{1}{2} \sigma^{2}\left(a^{i-1} s\right)^{2}} \\
& =\frac{1}{s} \sum_{n=1}^{\infty} e^{\sum_{i=1}^{n} \mu a^{i-1} s-\frac{1}{2} \sigma^{2}\left(a^{i-1} s\right)^{2}} \\
& =\frac{1}{S} \sum_{n=1}^{\infty} e^{\frac{\mu\left(1-a^{n}\right)}{(1-a)} s-\frac{\sigma^{2}\left(1-a^{2 n}\right) s^{2}}{\left(1-a^{2}\right)}} .
\end{aligned}
$$

Note that the argument of the summation is in the same form as the Laplace transform for the normal distribution. Given the uniqueness of the Laplace transform, equation 3.51 implies

$$
Q_{F}(t)=\sum_{n=1}^{\infty} \Phi\left[\frac{\mu\left(1-a^{n}\right)}{(1-a)}, \frac{\sigma^{2}\left(1-a^{2 n}\right)}{\left(1-a^{2}\right)}\right] .
$$

This confirms the result of Wang and Pham and shows that the quasi-renewal function is relatively straightforward to calculate in the case of the normal distribution.

\subsubsection{Uptime-Based Point Availability Function with Normal Repair}

Let the duration of the first repair interval be distributed as follows

$$
Y_{1} \sim \operatorname{normal}\left(\lambda, \rho^{2}\right) \rightarrow g_{Y_{1}}(t)=\frac{1}{\rho \sqrt{2 \pi}} e^{\frac{-(t-\lambda)^{2}}{2 \rho^{2}}}
$$


The Laplace transform of the density function of the $n^{\text {th }}$ repair interval is

$$
\begin{aligned}
& g_{Y_{1}}^{*}(s)=L^{-1}\left\{f_{Y_{1}}(t)\right\}=e^{\lambda s-\frac{1}{2} \rho^{2} s^{2}} \\
& g_{Y_{n}}^{*}(s)=g_{Y_{1}}^{*}\left(b^{n-1} s\right)=e^{\lambda b^{n-1} s-\frac{1}{2} \rho^{2}\left(b^{n-1} s\right)^{2}} .
\end{aligned}
$$

The uptime-based point availability function with normal quasi-renewal operating intervals and normal quasi-renewal repair intervals is given by

$$
\begin{aligned}
A^{*}(s)= & \frac{1}{s}\left[\left(1-f_{X_{1}}^{*}(s)\right)+\sum_{n=1}^{\infty}\left(1-f_{X_{n+1}}^{*}(s)\right) h_{\Theta_{n}}^{*}(s)\right] \\
= & \left.\frac{1}{s}\left[\left(1-e^{\mu s-\frac{1}{2} \sigma^{2} s^{2}}\right)+\sum_{n=1}^{\infty}\left[\left(1-e^{\mu a^{n} s-\frac{1}{2} \sigma^{2} a^{2 n} s^{2}}\right) \prod_{i=1}^{n} e^{\mu a^{i-1} s-\frac{1}{2} \sigma^{2} a^{2(i-1)} s^{2}+\lambda b^{i-1} s-\frac{1}{2} \rho^{2} b^{2(i-1)} s^{2}}\right]\right]\right] \\
= & \frac{1}{s}\left[\left(1-e^{\mu s-\frac{1}{2} \sigma^{2} s^{2}}\right)+\sum_{n=1}^{\infty}\left[\left(1-e^{\mu a^{n} s-\frac{1}{2} \sigma^{2} a^{2 n} s^{2}}\right) e^{\sum_{i=1}^{n} \mu a^{i-1} s-\frac{1}{2} \sigma^{2} a^{2(i-1)} s^{2}+\lambda b^{i-1} s-\frac{1}{2} \rho^{2} b^{2(i-1)} s^{2}}\right]\right] \\
= & \frac{1}{s}\left[\sum_{n=1}^{\infty}\left[\left(1-e^{\mu a^{n} s-\frac{1}{2} \sigma^{2} a^{2 n} s^{2}}\right) e^{\left(\frac{\mu\left(1-a^{n}\right)}{(1-a)}+\lambda \sum_{i=1}^{n} b^{i-1}\right) s-\left(\frac{\sigma^{2}\left(1-a^{2 n}\right)}{\left(1-a^{2}\right)}+\rho^{2} \sum_{i=1}^{n} b^{2(i-1)}\right) \frac{s^{2}}{2}}\right]\right] \\
= & \left.\frac{1}{s}\left[\left(1-e^{\mu s-\frac{1}{2} \sigma^{2} s^{2}}\right)+\sum_{n=1}^{\infty}\left[e^{\left(\frac{\mu\left(1-a^{n}\right)}{(1-a)}+\lambda \sum_{i=1}^{n} b^{i-1}\right) s-\left(\frac{\sigma^{2}\left(1-a^{2 n}\right)}{\left(1-a^{2}\right)}+\rho^{2} \sum_{i=1}^{n} b^{2(i-1)}\right) \frac{s^{2}}{2}}\right]-e^{\left(\mu a^{n}+\frac{\mu\left(1-a^{n}\right)}{(1-a)}+\lambda \sum_{i=1}^{n} b^{i-1}\right) s-\left(\sigma^{2} a^{2 n}+\frac{\sigma^{2}\left(1-a^{2 n}\right)}{\left(1-a^{2}\right)}+\rho^{2} \sum_{i=1}^{n} b^{2(i-1)}\right) \frac{s^{2}}{2}}\right]\right] .
\end{aligned}
$$

Given the uniqueness of the Laplace transform the following expression is an equivalent expression in the time domain:

$$
\begin{aligned}
& A_{H}(t)=1-\Phi\left(\mu, \sigma^{2}\right)+ \\
& \sum_{n=1}^{\infty}\left[\begin{array}{l}
\Phi\left(\frac{\mu\left(1-a^{n}\right)}{(1-a)}+\lambda \sum_{i=1}^{n} b^{i-1}, \frac{\sigma^{2}\left(1-a^{2 n}\right)}{\left(1-a^{2}\right)}+\rho^{2} \sum_{i=1}^{n} b^{2(i-1)}\right)- \\
\Phi\left(\mu a^{n}+\frac{\mu\left(1-a^{n}\right)}{(1-a)}+\lambda \sum_{i=1}^{n} b^{i-1}, \sigma^{2} a^{2 n}+\frac{\sigma^{2}\left(1-a^{2 n}\right)}{\left(1-a^{2}\right)}+\rho^{2} \sum_{i=1}^{n} b^{2(i-1)}\right)
\end{array}\right] .
\end{aligned}
$$


The uptime-based point availability function under iid repair with normal quasirenewal operating intervals and normal iid repair intervals is given by

$$
\begin{aligned}
A^{*}(s) & =\frac{1}{s}\left[\left(1-f_{X_{1}}^{*}(s)\right)+\sum_{n=1}^{\infty}\left(1-f_{X_{n+1}}^{*}(s)\right)\left(g_{Y_{1}}^{*}(s)\right)^{n} \prod_{i=1}^{n} f_{X_{i}}^{*}(s)\right] \\
& =\frac{1}{s}\left[\left(1-e^{\mu s-\frac{1}{2} \sigma^{2} s^{2}}\right)+\sum_{n=1}^{\infty}\left[\left(1-e^{\mu a^{n} s-\frac{1}{2} \sigma^{2} a^{2 n} s^{2}}\right) e^{n \lambda s-\frac{1}{2} n \rho^{2} s^{2}} \prod_{i=1}^{n} e^{\mu a^{i-1} s-\frac{1}{2} \sigma^{2} a^{2(i-1)} s^{2}}\right]\right] \\
& =\frac{1}{S}\left[\left(1-e^{\mu s-\frac{1}{2} \sigma^{2} s^{2}}\right)+\sum_{n=1}^{\infty}\left[\left(1-e^{\mu a^{n} s-\frac{1}{2} \sigma^{2} a^{2 n} s^{2}}\right) e^{n \lambda s-\frac{1}{2} n \rho^{2} s^{2}} e^{\sum_{i=1}^{n} \mu a^{i-1} s-\frac{1}{2} \sigma^{2} a^{2(i-1)} s^{2}}\right]\right] \\
& =\frac{1}{s}\left[\left(1-e^{\mu s-\frac{1}{2} \sigma^{2} s^{2}}\right)+\sum_{n=1}^{\infty}\left[\left(1-e^{\mu a^{n} s-\frac{1}{2} \sigma^{2} a^{2 n} s^{2}}\right) e^{n \lambda s-\frac{1}{2} n \rho^{2} s^{2}} e^{\frac{\mu\left(1-a^{n}\right)}{(1-a)} s-\frac{\sigma^{2}\left(1-a^{2 n}\right)}{\left(1-a^{2}\right)} s^{2}}\right]\right] \\
& \left.=\frac{1}{s}\right]\left[1-e^{\mu s-\frac{1}{2} \sigma^{2} s^{2}}\right]+\sum_{n=1}^{\infty}\left[e^{\left(\frac{\mu\left(1-a^{n}\right)}{(1-a)}+n \lambda\right) s-\left(\frac{\sigma^{2}\left(1-a^{2 n}\right)}{\left(1-a^{2}\right)}+n \rho^{2}\right) \frac{s^{2}}{2}}\right] .
\end{aligned}
$$

Given the uniqueness of the Laplace transform the following expression is an equivalent expression in the time domain:

$$
\begin{aligned}
& A_{H}(t)=1-\Phi\left(\mu, \sigma^{2}\right)+ \\
& \sum_{n=1}^{\infty}\left[\begin{array}{l}
\Phi\left(\frac{\mu\left(1-a^{n}\right)}{(1-a)}+n \lambda, \frac{\sigma^{2}\left(1-a^{2 n}\right)}{\left(1-a^{2}\right)}+n \rho^{2}\right)- \\
\Phi\left(\mu a^{n}+\frac{\mu\left(1-a^{n}\right)}{(1-a)}+n \lambda, \sigma^{2} a^{2 n}+\frac{\sigma^{2}\left(1-a^{2 n}\right)}{\left(1-a^{2}\right)}+n \rho^{2}\right)
\end{array}\right] .
\end{aligned}
$$




\subsubsection{Downtime-Based Point Availability Function with Normal Repair}

The downtime-based point availability function with normal quasi-renewal operating intervals and normal quasi-renewal repair intervals is given by

$$
\begin{aligned}
& A^{*}(s)=\frac{1}{s}\left[1-\sum_{n=1}^{\infty}\left(\frac{1}{g_{Y_{n}}^{*}(s)}-1\right) h_{\Theta_{n}}^{*}(s)\right] \\
& \left.=\frac{1}{S}\left[1-\sum_{n=1}^{\infty}\left(e^{\frac{1}{2} \rho^{2} b^{2 n-2} s^{2}-\lambda b^{n-1} s}-1\right) e^{\left(\frac{\mu\left(1-a^{n}\right)}{(1-a)}+\lambda \sum_{i=1}^{n} b^{i-1}\right) s-\left(\frac{\sigma^{2}\left(1-a^{2 n}\right)}{\left(1-a^{2}\right)}+\rho^{2} \sum_{i=1}^{n} b^{2(i-1)}\right)}\right) \frac{s^{2}}{2}\right]
\end{aligned}
$$

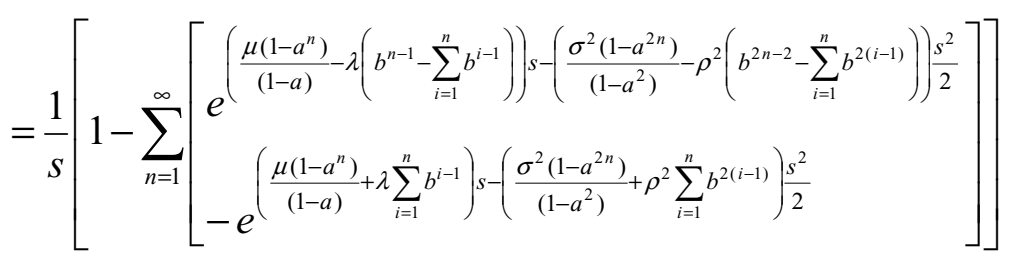

$$
\begin{aligned}
& =\frac{1}{s}\left[1-\sum_{n=1}^{\infty}\left[\begin{array}{c}
e^{\left(\frac{\mu\left(1-a^{n}\right)}{(1-a)}+\lambda \sum_{i=1}^{n-1} b^{i-1}\right) s-\left(\frac{\sigma^{2}\left(1-a^{2 n}\right)}{\left(1-a^{2}\right)}+\rho^{2} \sum_{i=1}^{n-1} b^{2(i-1)}\right) \frac{s^{2}}{2}} \\
-e^{\left(\frac{\mu\left(1-a^{n}\right)}{(1-a)}+\lambda \sum_{i=1}^{n} b^{i-1}\right) s-\left(\frac{\sigma^{2}\left(1-a^{2 n}\right)}{\left(1-a^{2}\right)}+\rho^{2} \sum_{i=1}^{n} b^{2(i-1)}\right) \frac{s^{2}}{2}}
\end{array}\right]\right]
\end{aligned}
$$

Given the uniqueness of the Laplace transform the following expression is an equivalent expression in the time domain:

$$
A_{H}(t)=1-\sum_{n=1}^{\infty}\left[\begin{array}{c}
\Phi\left(\frac{\mu\left(1-a^{n}\right)}{(1-a)}+\lambda \sum_{i=1}^{n-1} b^{i-1}, \frac{\sigma^{2}\left(1-a^{2 n}\right)}{\left(1-a^{2}\right)}+\rho^{2} \sum_{i=1}^{n-1} b^{2(i-1)}\right) \\
-\Phi\left(\frac{\mu\left(1-a^{n}\right)}{(1-a)}+\lambda \sum_{i=1}^{n} b^{i-1}, \frac{\sigma^{2}\left(1-a^{2 n}\right)}{\left(1-a^{2}\right)}+\rho^{2} \sum_{i=1}^{n} b^{2(i-1)}\right)
\end{array}\right] .
$$


The downtime-based point availability function under iid repair with normal quasirenewal operating intervals and normal iid repair intervals is given by

$$
\begin{aligned}
& A^{*}(s)=\frac{1}{s}\left[1-\left(\frac{1}{g_{Y_{1}}^{*}(s)}-1\right) \sum_{n=1}^{\infty} h_{\Theta_{n}}^{*}(s)\right] \\
&=\frac{1}{s}\left[1-\left(e^{\frac{1}{2} \rho^{2} s^{2}-\lambda s}-1\right) \sum_{n=1}^{\infty} e^{n\left(\lambda s-\frac{1}{2} \rho^{2} s^{2}\right)} e^{\frac{\mu\left(1-a^{n}\right)}{(1-a)} s-\frac{\sigma^{2}\left(1-a^{2 n}\right)}{\left(1-a^{2}\right)} \frac{s^{2}}{2}}\right] \\
&=\frac{1}{s}\left[1-\left(e^{\frac{1}{2} \rho^{2} s^{2}-\lambda s}-1\right) \sum_{n=1}^{\infty} e^{\left(n \lambda+\frac{\mu\left(1-a^{n}\right)}{(1-a)}\right) s-\left(n \rho^{2}+\frac{\sigma^{2}\left(1-a^{2 n}\right)}{\left(1-a^{2}\right)}\right) \frac{s^{2}}{2}}\right] \\
&=\frac{1}{s}\left[1-\left(e^{\frac{1}{2} \rho^{2} s^{2}-\lambda s}-1\right) \sum_{n=1}^{\infty} e^{\left(n \lambda+\frac{\mu\left(1-a^{n}\right)}{(1-a)}\right) s-\left(n \rho^{2}+\frac{\sigma^{2}\left(1-a^{2 n}\right)}{\left(1-a^{2}\right)}\right) \frac{s^{2}}{2}}\right] \\
&=\frac{1}{s}-\frac{1}{s}\left(\sum_{n=1}^{\infty} e^{\left((n-1) \lambda+\frac{\mu\left(1-a^{n}\right)}{(1-a)}\right) s-\left((n-1) \rho^{2}+\frac{\sigma^{2}\left(1-a^{2 n}\right)}{\left(1-a^{2}\right)}\right) \frac{s^{2}}{2}}\right] \\
&\left.-\sum_{n=1}^{\infty} e^{\left(n \lambda+\frac{\mu\left(1-a^{n}\right)}{(1-a)}\right) s-\left(n \rho^{2}+\frac{\sigma^{2}\left(1-a^{2 n}\right)}{\left(1-a^{2}\right)}\right) \frac{s^{2}}{2}}\right] .
\end{aligned}
$$

Given the uniqueness of the Laplace transform the following expression is an equivalent expression in the time domain:

$$
A_{H}(t)=1-\sum_{n=1}^{\infty}\left[\begin{array}{l}
\Phi\left((n-1) \lambda+\frac{\mu\left(1-a^{n}\right)}{(1-a)},(n-1) \rho^{2}+\frac{\sigma^{2}\left(1-a^{2 n}\right)}{\left(1-a^{2}\right)}\right) \\
-\Phi\left(n \lambda+\frac{\mu\left(1-a^{n}\right)}{(1-a)}, n \rho^{2}+\frac{\sigma^{2}\left(1-a^{2 n}\right)}{\left(1-a^{2}\right)}\right)
\end{array}\right] .
$$

\subsubsection{Uptime-Based Point Availability Function with Exponential Repair}

Let the duration of the first repair interval be distributed as follows

$$
Y_{1} \sim \exp (\lambda) \rightarrow g_{Y_{1}}(t)=\lambda e^{-\lambda t} \text { for } t>0 .
$$


The uptime-based point availability function with normal quasi-renewal operating intervals and exponential quasi-renewal repair intervals is given by

$$
\begin{aligned}
& A^{*}(s)= \frac{1}{s}\left[\left(1-f_{X_{1}}^{*}(s)\right)+\sum_{n=1}^{\infty}\left(1-f_{X_{n+1}}^{*}(s)\right) h_{\Theta_{n}}^{*}(s)\right] \\
&= \frac{1}{s}\left[\left(1-e^{\mu s-\frac{1}{2} \sigma^{2} s^{2}}\right)+\sum_{n=1}^{\infty}\left[\left(1-e^{\mu a^{n} s-\frac{1}{2} \sigma^{2} a^{2 n} s^{2}}\right) \prod_{i=1}^{n}\left(\frac{\lambda}{\lambda+b^{i-1} s}\right) e^{\mu a^{i-1} s-\frac{1}{2} \sigma^{2} a^{2(i-1)} s^{2}}\right]\right] \\
&= \frac{1}{s}\left[\left(1-e^{\mu s-\frac{1}{2} \sigma^{2} s^{2}}\right)+\sum_{n=1}^{\infty}\left[\left(1-e^{\mu a^{n} s-\frac{1}{2} \sigma^{2} a^{2 n} s^{2}}\right) e^{\sum_{i=1}^{n} \mu a^{i-1} s-\frac{1}{2} \sigma^{2} a^{2(i-1)} s^{2}} \prod_{i=1}^{n}\left(\frac{\lambda}{\lambda+b^{i-1} s}\right)\right]\right] \\
&= \frac{1}{s}\left[\left(1-e^{\mu s-\frac{1}{2} \sigma^{2} s^{2}}\right)\right] \\
&\left.+\sum_{n=1}^{\infty}\left[\left(1-e^{\mu a^{n} s-\frac{1}{2} \sigma^{2} a^{2 n} s^{2}}\right) e^{\left(\frac{\mu\left(1-a^{n}\right)}{(1-a)}\right) s-\left(\frac{\sigma^{2}\left(1-a^{2 n}\right)}{\left(1-a^{2}\right)}\right) \frac{s^{2}}{2}} \prod_{i=1}^{n}\left(\frac{\lambda}{\lambda+b^{i-1} s}\right)\right]\right]
\end{aligned}
$$

The uptime-based point availability function under iid repair with normal quasirenewal operating intervals and exponential iid repair intervals is given by

$$
\begin{aligned}
A^{*}(s) & =\frac{1}{s}\left[\left(1-f_{X_{1}}^{*}(s)\right)+\sum_{n=1}^{\infty}\left(1-f_{X_{n+1}}^{*}(s)\right) h_{\Theta_{n}}^{*}(s)\right] \\
& =\frac{1}{s}\left[\left(1-e^{\mu s-\frac{1}{2} \sigma^{2} s^{2}}\right)+\sum_{n=1}^{\infty}\left[\left(1-e^{\mu a^{n} s-\frac{1}{2} \sigma^{2} a^{2 n} s^{2}}\right) \prod_{i=1}^{n}\left(\frac{\lambda}{\lambda+s}\right) e^{\mu a^{i-1} s-\frac{1}{2} \sigma^{2} a^{2(i-1)} s^{2}}\right]\right] \\
& =\frac{1}{s}\left[\left(1-e^{\mu s-\frac{1}{2} \sigma^{2} s^{2}}\right)+\sum_{n=1}^{\infty}\left[\left(1-e^{\mu a^{n} s-\frac{1}{2} \sigma^{2} a^{2 n} s^{2}}\right)\left(\frac{\lambda}{\lambda+s}\right)^{n} e^{\sum_{i=1}^{n} \mu a^{i-1} s-\frac{1}{2} \sigma^{2} a^{2(i-1)} s^{2}}\right]\right] \\
& =\frac{1}{s}\left[\left(1-e^{\mu s-\frac{1}{2} \sigma^{2} s^{2}}\right)+\sum_{n=1}^{\infty}\left[\left(\frac{\lambda}{\lambda+s}\right)^{n}\left(1-e^{\mu a^{n} s-\frac{1}{2} \sigma^{2} a^{2 n} s^{2}}\right) e^{\left(\frac{\mu\left(1-a^{n}\right)}{(1-a)}\right) s-\left(\frac{\sigma^{2}\left(1-a^{2 n}\right)}{\left(1-a^{2}\right)}\right) \frac{s^{2}}{2}}\right]\right] .
\end{aligned}
$$


3.7.5 Downtime-Based Point Availability Function with Exponential Repair

The downtime-based point availability function with normal quasi-renewal operating intervals and exponential quasi-renewal repair intervals is given by

$$
\begin{aligned}
A^{*}(s) & =\frac{1}{s}\left[1-\sum_{n=1}^{\infty}\left(\frac{1}{g_{Y_{n}}^{*}(s)}-1\right) h_{\Theta_{n}}^{*}(s)\right] \\
& =\frac{1}{s}\left[1-\sum_{n=1}^{\infty}\left(\frac{\lambda+b^{n-1} s}{\lambda}-1\right) e^{\frac{\mu\left(1-a^{n}\right)}{(1-a)} s-\frac{\sigma^{2}\left(1-a^{2 n}\right) s^{2}}{\left(1-a^{2}\right)}{ }^{2}} \prod_{i=1}^{n} \frac{\lambda}{\lambda+b^{i-1} s}\right] \\
& =\frac{1}{s}-\sum_{n=1}^{\infty}(b \lambda)^{n-1} e^{\frac{\mu\left(1-a^{n}\right)}{(1-a)} s-\frac{\sigma^{2}\left(1-a^{2 n}\right) s^{2}}{\left(1-a^{2}\right)}} \prod_{i=1}^{n} \frac{1}{\lambda+b^{i-1} s} .
\end{aligned}
$$

The downtime-based point availability function under iid repair with normal quasirenewal operating intervals and exponential iid repair intervals is given by

$$
\begin{aligned}
A^{*}(s) & =\frac{1}{s}\left[1-\left(\frac{1}{g_{Y_{1}}^{*}(s)}-1\right) \sum_{n=1}^{\infty} h_{\Theta_{n}}^{*}(s)\right] \\
& =\frac{1}{s}\left[1-\left(\frac{\lambda+s}{\lambda}-1\right) \sum_{n=1}^{\infty}\left(\frac{\lambda}{\lambda+s}\right)^{n} e^{\frac{\mu\left(1-a^{n}\right)}{(1-a)} s-\frac{\sigma^{2}\left(1-a^{2 n}\right) s^{2}}{\left(1-a^{2}\right)} 2}\right] \\
& =\frac{1}{s}-\frac{1}{\lambda} \sum_{n=1}^{\infty}\left(\frac{\lambda}{\lambda+s}\right)^{n} e^{\frac{\mu\left(1-a^{n}\right)}{(1-a)} s-\frac{\sigma^{2}\left(1-a^{2 n}\right) s^{2}}{\left(1-a^{2}\right)} 2}
\end{aligned}
$$

The point availability functions under normal operating intervals and normal repair intervals are easily expressed in the time domain. However, the inversion of the point availability functions under normal operating intervals and exponential repair intervals is not as straightforward.

\subsection{Quasi-Renewal Model Under Gamma Operating Intervals}

The quasi-renewal function is constructed and is the foundation for the development of the availability expressions under gamma distributed operating intervals. Uptimebased and downtime-based point availability functions are constructed under the 
assumption of gamma distributed quasi-renewal operating intervals for both the quasirenewal repair case and the iid repair case. The repair intervals are modeled using both the gamma distribution and the exponential distribution. Inversion of the exact transform expression is not possible without truncation. Therefore the exact expression in the transform space is provided rather than an approximate expression in the time domain. Let the duration of the first interval of operation be distributed as follows

$$
X_{1} \sim \operatorname{gamma}(k, \lambda) \rightarrow f_{X_{1}}(t)=\frac{\lambda e^{-\lambda t}(\lambda t)^{k-1}}{(k-1) !} \text { for } t>0 .
$$

The Laplace transform of the density function of the $n^{\text {th }}$ operation interval is

$$
\begin{aligned}
& f_{X_{1}}^{*}(s)=L^{-1}\left\{f_{X_{1}}(t)\right\}=\left(\frac{\lambda}{\lambda+s}\right)^{k} \\
& f_{X_{n}}^{*}(s)=f_{X_{1}}^{*}\left(a^{n-1} s\right)=\left(\frac{\lambda}{\lambda+a^{n-1} s}\right)^{k} .
\end{aligned}
$$

\subsubsection{Gamma Quasi-Renewal Function}

The quasi-renewal function for gamma distributed operating intervals is given by

$$
Q^{*}(s)=\frac{1}{s} \sum_{n=1}^{\infty} \prod_{i=1}^{n} f_{X_{1}}^{*}\left(a^{i-1} s\right)=\frac{1}{s} \sum_{n=1}^{\infty} \lambda^{k n} \prod_{i=1}^{n} \frac{1}{\left(\lambda+a^{i-1} s\right)^{k}}
$$

\subsubsection{Uptime-Based Point Availability Function with Gamma Repair}

Let the duration of the first repair interval be distributed as follows

$$
Y_{1} \sim \operatorname{gamma}(r, \mu) \rightarrow g_{Y_{1}}(t)=\frac{\mu e^{-\mu t}(\mu t)^{r-1}}{(r-1) !} \text { for } t>0 .
$$

The Laplace transform of the density function of the $n^{\text {th }}$ repair interval is

$$
\begin{aligned}
& g_{Y_{1}}^{*}(s)=L^{-1}\left\{g_{Y_{1}}(t)\right\}=\left(\frac{\mu}{\mu+s}\right)^{r} \\
& g_{Y_{n}}^{*}(s)=g_{Y_{1}}^{*}\left(b^{n-1} s\right)=\left(\frac{\mu}{\mu+b^{n-1} s}\right)^{\mathrm{r}} .
\end{aligned}
$$


The uptime-based point availability function with gamma quasi-renewal operating intervals and gamma quasi-renewal repair intervals is given by

$$
\begin{aligned}
A^{*}(s) & =\frac{1}{s}\left[\left(1-f_{X_{1}}^{*}(s)\right)+\sum_{n=1}^{\infty}\left(1-f_{X_{n+1}^{*}}^{*}(s)\right) h_{\Theta_{n}}^{*}(s)\right] \\
& =\frac{1}{s}\left[\left(1-\left(\frac{\lambda}{\lambda+s}\right)^{k}\right)+\sum_{n=1}^{\infty}\left(1-\left(\frac{\lambda}{\lambda+a^{n} s}\right)^{k}\right) \prod_{i=1}^{n}\left(\frac{\lambda}{\lambda+a^{i-1} s}\right)^{k}\left(\frac{\mu}{\mu+b^{i-1} s}\right)^{r}\right] \\
& =\frac{1}{s}\left[\left(1-\left(\frac{\lambda}{\lambda+s}\right)^{k}\right)+\sum_{n=1}^{\infty}\left(1-\left(\frac{\lambda}{\lambda+a^{n} s}\right)^{k}\right)\left(\lambda^{k} \mu^{r}\right)^{n} \prod_{i=1}^{n} \frac{1}{\left(\lambda+a^{i-1} s\right)^{k}\left(\mu+b^{i-1} s\right)^{r}}\right]
\end{aligned}
$$

The uptime-based point availability function under iid repair with gamma quasirenewal operating intervals and gamma iid repair intervals is given by

$$
\begin{aligned}
A^{*}(s) & =\frac{1}{s}\left[\left(1-f_{X_{1}}^{*}(s)\right)+\sum_{n=1}^{\infty}\left(1-f_{X_{n+1}}^{*}(s)\right) h_{\Theta_{n}}^{*}(s)\right] \\
& =\frac{1}{s}\left[\left(1-\left(\frac{\lambda}{\lambda+s}\right)^{k}\right)+\sum_{n=1}^{\infty}\left(1-\left(\frac{\lambda}{\lambda+a^{n} s}\right)^{k}\right)\left(\frac{\mu}{\mu+s}\right)^{n r} \prod_{i=1}^{n}\left(\frac{\lambda}{\lambda+a^{i-1} s}\right)^{k}\right] \\
& =\frac{1}{S}\left[\left(1-\left(\frac{\lambda}{\lambda+s}\right)^{k}\right)+\sum_{n=1}^{\infty}\left(1-\left(\frac{\lambda}{\lambda+a^{n} s}\right)^{k}\right)\left(\frac{\mu}{\mu+s}\right)^{n r} \lambda^{k n} \prod_{i=1}^{n} \frac{1}{\left(\lambda+a^{i-1} s\right)^{k}}\right]
\end{aligned}
$$

\subsubsection{Downtime-Based Point Availability Function with Gamma Repair}

The downtime-based point availability function with gamma quasi-renewal operating intervals and gamma quasi-renewal repair intervals is given by

$$
\begin{aligned}
A^{*}(s) & =\frac{1}{s}\left[1-\sum_{n=1}^{\infty}\left(\frac{1}{g_{Y_{n}}^{*}(s)}-1\right) h_{\Theta_{n}}^{*}(s)\right] \\
& =\frac{1}{S}\left[1-\sum_{n=1}^{\infty}\left(\left(\frac{\mu+b^{n-1} s}{\mu}\right)^{r}-1\right)\left(\lambda^{k} \mu^{r}\right)^{n} \prod_{i=1}^{n} \frac{1}{\left(\lambda+a^{i-1} s\right)^{k}\left(\mu+b^{i-1} s\right)^{r}}\right]
\end{aligned}
$$


The downtime-based point availability function under iid repair with gamma quasirenewal operating intervals and gamma iid repair intervals is given by

$$
\begin{aligned}
A^{*}(s) & =\frac{1}{s}\left[1-\left(\frac{1}{g_{Y_{1}}^{*}(s)}-1\right) \sum_{n=1}^{\infty} h_{\Theta_{n}}^{*}(s)\right] \\
& =\frac{1}{s}\left[1-\left(\left(\frac{\mu+s}{\mu}\right)^{r}-1\right) \sum_{n=1}^{\infty}\left(\frac{\mu}{\mu+s}\right)^{n r} \lambda^{k n} \prod_{i=1}^{n} \frac{1}{\left(\lambda+a^{i-1} s\right)^{k}}\right]
\end{aligned}
$$

\subsubsection{Uptime-Based Point Availability Function with Exponential Repair}

Let the duration of the first repair interval be distributed as follows

$$
Y_{1} \sim \exp (\mu) \rightarrow g_{Y_{1}}(t)=\mu e^{-\mu t} \text { for } t>0 .
$$

The uptime-based point availability function with gamma quasi-renewal operating intervals and exponential quasi-renewal repair intervals is given by

$$
\begin{aligned}
A^{*}(s) & =\frac{1}{s}\left[\left(1-f_{X_{1}}^{*}(s)\right)+\sum_{n=1}^{\infty}\left(1-f_{X_{n+1}}^{*}(s)\right) h_{\Theta_{n}}^{*}(s)\right] \\
& =\frac{1}{s}\left[\left(1-\left(\frac{\lambda}{\lambda+s}\right)^{k}\right)+\sum_{n=1}^{\infty}\left(1-\left(\frac{\lambda}{\lambda+a^{n} s}\right)^{k}\right) \prod_{i=1}^{n}\left(\frac{\lambda}{\lambda+a^{i-1} s}\right)^{k}\left(\frac{\mu}{\mu+b^{i-1} s}\right)\right] \\
& =\frac{1}{s}\left[\left(1-\left(\frac{\lambda}{\lambda+s}\right)^{k}\right)+\sum_{n=1}^{\infty}\left(1-\left(\frac{\lambda}{\lambda+a^{n} s}\right)^{k}\right)\left(\mu \lambda^{k}\right)^{n} \prod_{i=1}^{n} \frac{1}{\left(\lambda+a^{i-1} s\right)^{k}\left(\mu+b^{i-1} s\right)}\right]
\end{aligned}
$$

The uptime-based point availability function under iid repair with gamma quasirenewal operating intervals and exponential iid repair intervals is given by 


$$
\begin{aligned}
A^{*}(s) & =\frac{1}{s}\left[\left(1-f_{X_{1}}^{*}(s)\right)+\sum_{n=1}^{\infty}\left(1-f_{X_{n+1}}^{*}(s)\right) h_{\Theta_{n}}^{*}(s)\right] \\
& =\frac{1}{s}\left[\left(1-\left(\frac{\lambda}{\lambda+s}\right)^{k}\right)+\sum_{n=1}^{\infty}\left(1-\left(\frac{\lambda}{\lambda+a^{n} s}\right)^{k}\right)\left(\frac{\mu}{\mu+s}\right)^{n} \prod_{i=1}^{n}\left(\frac{\lambda}{\lambda+a^{i-1} s}\right)^{k}\right] \\
& =\frac{1}{s}\left[\left(1-\left(\frac{\lambda}{\lambda+s}\right)^{k}\right)+\sum_{n=1}^{\infty}\left(1-\left(\frac{\lambda}{\lambda+a^{n} s}\right)^{k}\right)\left(\frac{\mu}{\mu+s}\right)^{n} \lambda^{k n} \prod_{i=1}^{n} \frac{1}{\left(\lambda+a^{i-1} s\right)^{k}}\right]
\end{aligned}
$$

\subsubsection{Downtime-Based Point Availability Function with Exponential Repair}

The downtime-based point availability function with gamma quasi-renewal operating intervals and exponential quasi-renewal repair intervals is given by

$$
\begin{aligned}
A^{*}(s) & =\frac{1}{s}\left[1-\sum_{n=1}^{\infty}\left(\frac{1}{g_{Y_{n}}^{*}(s)}-1\right) h_{\Theta_{n}}^{*}(s)\right] \\
& =\frac{1}{s}-\frac{1}{s} \sum_{n=1}^{\infty}\left(\frac{\mu+b^{n-1} s}{\mu}-1\right)\left(\mu \lambda^{k}\right)^{n} \prod_{i=1}^{n} \frac{1}{\left(\lambda+a^{i-1} s\right)^{k}\left(\mu+b^{i-1} s\right)} \\
& =\frac{1}{s}-\sum_{n=1}^{\infty}(\mu b)^{n-1} \lambda^{k n} \prod_{i=1}^{n} \frac{1}{\left(\lambda+a^{i-1} s\right)^{k}\left(\mu+b^{i-1} s\right)}
\end{aligned}
$$

The downtime-based point availability function under iid repair with gamma quasirenewal operating intervals and exponential iid repair intervals is given by

$$
\begin{aligned}
A^{*}(s) & =\frac{1}{s}\left[1-\left(\frac{1}{g_{Y_{1}}^{*}(s)}-1\right) \sum_{n=1}^{\infty} h_{\Theta_{n}}^{*}(s)\right] \\
& =\frac{1}{s}-\frac{1}{\mu} \sum_{n=1}^{\infty}\left(\frac{\mu}{\mu+s}\right)^{n} \lambda^{k n} \prod_{i=1}^{n} \frac{1}{\left(\lambda+a^{i-1} s\right)^{k}}
\end{aligned}
$$

\subsection{Summary}

The Laplace transforms of the uptime-based and downtime-based point availability functions are found for the quasi-renewal process. The two forms of the point availability are based on complementary events and are equivalent. The Laplace 
transform must be inverted from the transform space to the time domain in order to analyze time-dependent system behavior. When the operating intervals and repair intervals both follow a normal distribution the inversion is straightforward. However, in the other cases explored the infinite sum in the transform space must be truncated before inversion to the time domain is possible. Truncation of the transform expression leads to an approximate expression in the time domain after inversion. The computational difficulty of approximating the exact expressions developed in this chapter is discussed in Chapter 4. Although the transform expressions of the uptime-based and downtime-based point availability are exact and equivalent, after truncation and inversion they provide two different approximations in the time domain. The uptime-based availability function provides a lower bound on the point availability while the downtime-based availability function provides an upper bound on the point availability. This property is useful in identifying the error in point availability calculations and in choosing the point of truncation necessary to achieve a desired degree of accuracy in such calculations. In addition, it appears that this development may be applicable in a broader context than that of just the quasi-renewal process. These general results in 3.29 and 3.32 may be useful in determining the availability behavior of any system that is characterized as having independent and unique operating intervals and repair intervals.

Several specific point availability expressions under various assumptions regarding the distribution of operating intervals and repair intervals are contained above. The timedependent system behavior of each of the cases is examined in the next chapter. Graphical and numerical results are obtained and the observed behavior is discussed. 


\section{Chapter 4 - Analysis and Discussion}

The quasi-renewal function and two point availability functions are developed for several distributions on operating time and repair time in the previous chapter. This chapter will explore the time-dependent behavior of these specific models. Each model is characterized by a set of quantities that correspond to the deterioration of the operation intervals, the deterioration of the repair intervals, the parameters of the theoretical distribution on the intervals of operation, and the parameters of the theoretical distribution on the intervals of repair. The impact that each of these quantities has on the behavior of the point availability function is described. Particularly, an effort is made to address the sensitivity of the point availability to each of these quantities. It is assumed that the mean of the distribution on the operation intervals is simply a measure of the scale on which behavior is observed. More importantly, consideration is given to the impact of the ratio of the mean of the first repair interval to the mean of the first operation interval, the degree of deterioration of the operation intervals, the degree of deterioration of the repair intervals, and the size of the variance relative to the mean of both the repair and operation intervals.

Issues related to the truncation necessary to invert the exact Laplace transforms and obtain numerical results are discussed. Truncation of the transform expression has an impact on both the quasi-renewal function and the point availability function. In addition, the behavior of the truncated uptime-based and downtime-based point availability functions is explored as they are used to bound the true availability.

\subsection{Computation}

In the case where both the operating intervals and repair intervals follow a normal distribution the computation of the quasi-renewal function and the point availability functions is straightforward. All of the relevant functions are expressed in the time domain and only require the ability to approximate the cumulative distribution function of the normal distribution. This being the case, the computation time is relatively small 
and accurate representations of all relevant expressions are obtained for the useful life of the system. For this reason, it is a worthwhile future endeavor to investigate the conditions under which other theoretical distributions on operating intervals and repair intervals can be adequately approximated with the normal distribution for analysis in the quasi-renewal framework.

In all cases other than the one having normal operating and repair times, the approximation of the quasi-renewal function and the point availability functions requires the inversion of a truncated infinite sum in the Laplace transform space. The Laplace transform expressions are exact but become approximations after they are truncated for the purpose of inversion. The number of terms that are inverted, $c$, and the degree of precision in machine-level arithmetic determines the degree of accuracy of the approximation. The sensitivity of these expressions to computational error necessitates that the Laplace transforms are inverted symbolically. Even when inversion is performed symbolically the complexity and nature of the resulting expressions cause machine level rounding error to be a significant limitation in the results that can be obtained. Computational intensity increases significantly for each additional term that is inverted. Therefore, computing power is also a significant limiting factor on the attainable degree of accuracy. Inversion was performed using Mathematica Version 4 on a PC with an 800-megahertz processor and 500 megabytes of RAM. With this equipment it is possible to compute the quasi-renewal functions for the gamma and exponential distributions by inverting the first 20 terms in the infinite sum. The point availability functions are more demanding and could only be approximated by inverting the first 10 to 13 terms for exponentially distributed repair and the first 7 terms for gamma distributed repair. A sample of the Mathematica input and output is provided in Appendix A and Appendix B.

The inversion of the terms in the point availability function based on normal operating intervals and exponential repair intervals is not possible using a conventional software package. Each term has the base of the natural log in the numerator with an exponent that is a function of the transform variable, $s$ and $s^{2}$. The denominator is an 
increasingly complex polynomial in $s$. Another method was not found to invert a Laplace transform of this particular form.

It is important to recognize that the numerical work performed to generate the graphical representations of the quasi-renewal and point availability functions is not exact and possesses a degree of inherent error. Calculations are limited by the machine precision with which internal computer calculations are made and the fact that the exact expressions themselves must be truncated to obtain numerical results. Due to the nature of the expressions in the time domain, the imprecision of machine numbers adds an additional source of error to the calculation of the approximated functions themselves.

The error in numerical analysis plays an important role in this work since some of the inverted Laplace transforms require calculations to be made involving several terms that include $a^{x}$ or $\frac{1}{a^{x}}$ for an extremely large $x$. Since $0<a<1$ and the value of $x$ exceeds 100 regularly, this results in some numbers that are smaller or larger than the set of machine numbers. This does not occur when operating intervals are normally distributed since the inverted form of all the expressions only requires the evaluation of the normal cumulative distribution function. Some of this behavior is seen in the terms corresponding to the larger values of $c$ for exponential operating intervals, where $\mathrm{c}$ is the number of terms of the infinite sum that are evaluated. However, in the case of exponential operating times there is no impact on the qualitative observations made and negligible impact on the numerical results. The frequency and intensity of this occurrence in calculations with gamma operating and repair intervals limited the amount of numerical results obtained. It is only possible to obtain meaningful numerical results for gamma operating intervals when the shape parameter of the gamma distribution is less than 4. Further discussion of this behavior is contained later in this chapter. 


\subsection{Impact of Truncating the Quasi-Renewal Function}

In order to obtain numerical results in the time domain for the quasi-renewal functions and the point availability functions of the models with exponential and gamma intervals of operation it is necessary to truncate the exact expressions. In all but one case the Laplace transform of the quasi-renewal function or the point availability must be truncated before it is inverted to the time domain. The exception is in the case of normal operating intervals and normal repair intervals. Under this condition the quasi-renewal function and the point availability functions are stated as infinite sums of normal cumulative distribution functions that must also be truncated to obtain numerical results. Although the quasi-renewal function for normal operating intervals is stated differently, the impact of truncation on the quasi-renewal function is the same in all of these cases. Similarly, the impact that truncation has on the point availability functions is the same in all cases.

The quasi-renewal function represents the expected number of restarts over time under the assumption that repair times are negligible. In other words, the expected number of operation intervals that are been completed at any point in time. Each term of the infinite sum in the expression for the quasi-renewal function,

$$
Q_{F}(t)=L^{-1}\left\{\frac{1}{s} \sum_{n=1}^{\infty} \prod_{i=1}^{n} f_{\Omega_{n}}^{*}(s)\right\}=\sum_{n=1}^{\infty} F_{\Omega_{n}}(t),
$$

corresponds to the probability that there are $n$ completed operation intervals by time $t$. This statement is true for each term in either the Laplace transform expression or the expression in the time domain. The sum to infinity is equal to the expected value of the number of completed operation intervals at time $t$.

When equation 4.1 is truncated at a point $c$,

$$
Q_{F}(t)=L^{-1}\left\{\frac{1}{s} \sum_{n=1}^{c} \prod_{i=1}^{n} f_{\Omega_{n}}^{*}(s)\right\}=\sum_{n=1}^{c} F_{\Omega_{n}}(t),
$$

this effectively limits the possible number of completed operation intervals under consideration. As a result, the quasi-renewal function asymptotically approaches $c$ in a 
deteriorating system. An example of this behavior for normal operating intervals and exponential operating intervals is shown in Figures 4.1 and 4.2 respectively.

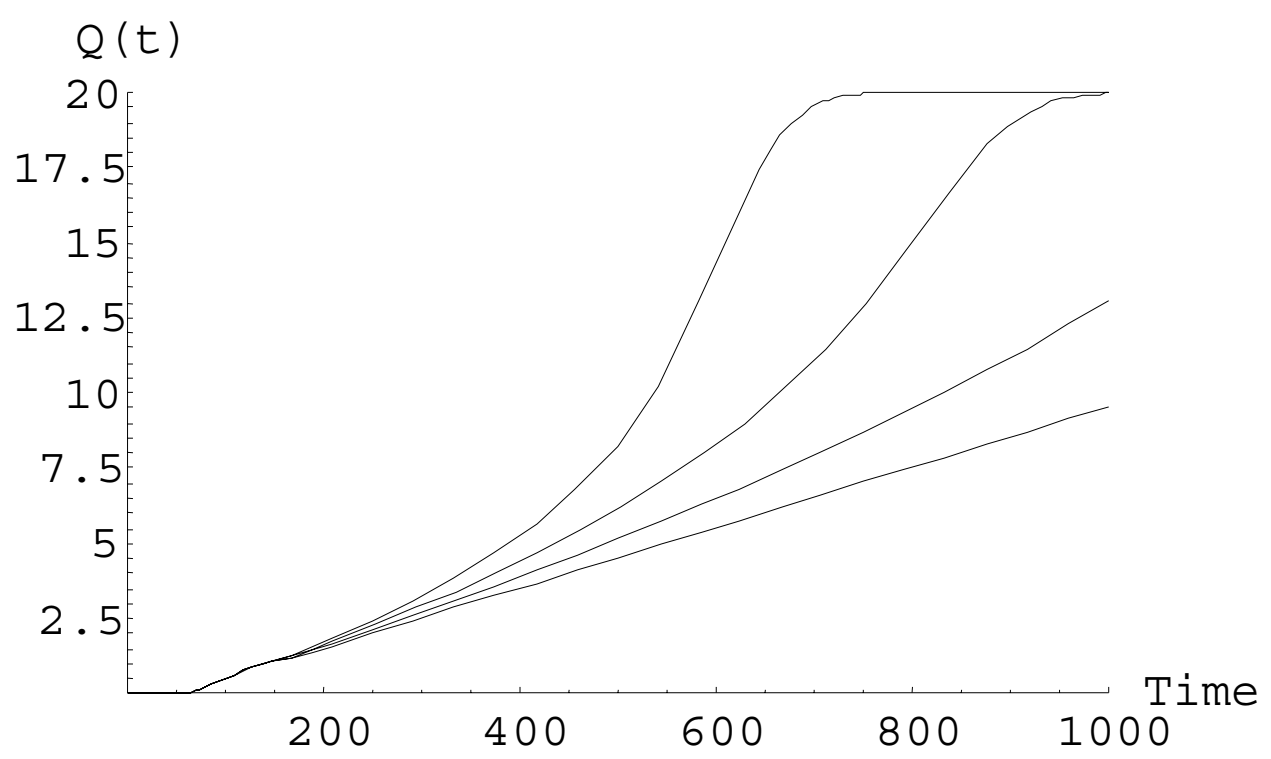

Figure 4.1 Normal $Q_{F}(t)$ with $\left.(\mu=100, \sigma=25, a=\{0.85,0.90,0.95,1.00)\}, c=20\right)$

In Figure 4.1 the first 20 terms in the infinite sum are evaluated so the quasi-renewal function for all cases will asymptotically approach 20 . It is clear that for systems that deteriorate quickly, $a=0.85$, the limit on the quasi-renewal function is reached earlier in the evolution of the process than systems that do not deteriorate as quickly, $a=0.9$ and $a=1.00$. In fact the approximation for the quasi-renewal functions with $a=0.9$ and $a=1.00$ is still adequate at 1000 time units. However, it is clear that at 1000 time units the approximation is rather poor for $a=0.8$ and $a=0.85$. An example of the identical behavior for the truncated quasi-renewal function with exponential operating intervals is shown below. 


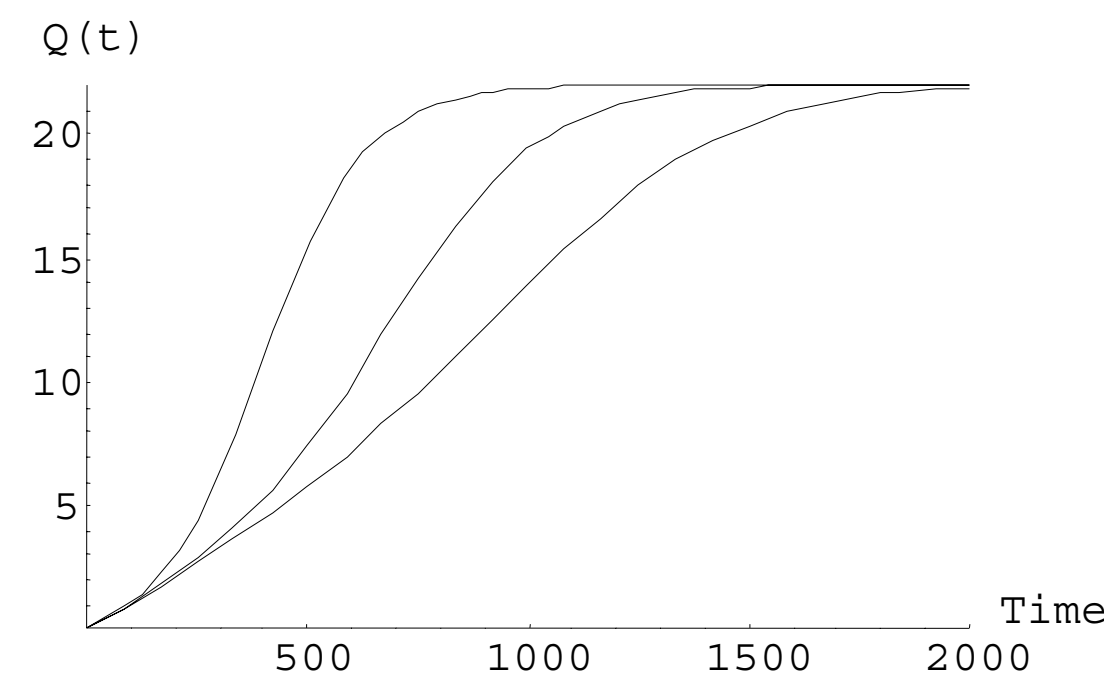

Figure 4.2 Exponential $Q_{F}(t)$ with $\left.(\lambda=0.01, a=\{0.80,0.90,0.95\}), c=22\right)$

The accuracy of the approximation of the quasi-renewal function increases in $c$. The value of $c$ necessary to adequately approximate the quasi-renewal function depends on the true expected number of restarts at the time of interest. If $c$ is less than or near the true expected number of restarts then the approximation will be poor since it starts to asymptotically approach the limiting value, $c$. The approximated function is accurate when the value of $c$ is much larger than the true expected value of the number of restarts. For any deteriorating system the approximation of the quasi-renewal function is more accurate for smaller vales of $t$ since the expected number of restarts is closer to its minimum.

\subsection{Impact of Truncating the Point Availability Expressions}

As discussed in section 3.5.3, the uptime-based and downtime-based point availability functions are used to bound the value of the true point availability. The uptime-based point availability function serves as the lower bound and the downtimebased point availability function serves as the upper bound. The fact that the two point availability expressions can be used to form bounds on the point availability is a direct result of the behavior of these expressions after truncation. The exact expressions for the point availability are equivalent, 


$$
A^{*}(s)=\frac{1}{s}\left[\left(1-f_{X_{1}}^{*}(s)\right)+\sum_{n=1}^{\infty}\left(1-f_{X_{n+1}}^{*}(s)\right) h_{\Theta_{n}}^{*}(s)\right]=\frac{1}{s}\left[1-\sum_{n=1}^{\infty}\left(\frac{1}{g_{Y_{n}}^{*}(s)}-1\right) h_{\Theta_{n}}^{*}(s)\right] .
$$

However,

$$
A_{H}(t)_{\text {Lower }}=L^{-1}\left\{A^{*}(s)_{\text {Lower }}\right\}=L^{-1}\left\{\frac{1}{s}\left[\left(1-f_{X_{1}}^{*}(s)\right)+\sum_{n=1}^{c}\left(1-f_{X_{n+1}}^{*}(s)\right) h_{\Theta_{n}}^{*}(s)\right]\right\}
$$

is a lower bound constructed with the first $c$ terms and

$$
A_{H}(t)_{U p p e r}=L^{-1}\left\{A^{*}(s)_{U p p e r}\right\}=L^{-1}\left\{\frac{1}{s}\left[1-\sum_{n=1}^{c}\left(\frac{1}{g_{Y_{n}}^{*}(s)}-1\right) h_{\Theta_{n}}^{*}(s)\right]\right\}
$$

is an upper bound constructed with the first $c$ terms.

Each term in the infinite sum of the lower bound corresponds to the event that the $n^{\text {th }}$ repair is finished at time $u$ and the $(n+1)^{\text {st }}$ operation interval is of length $(t-u)$ or greater.

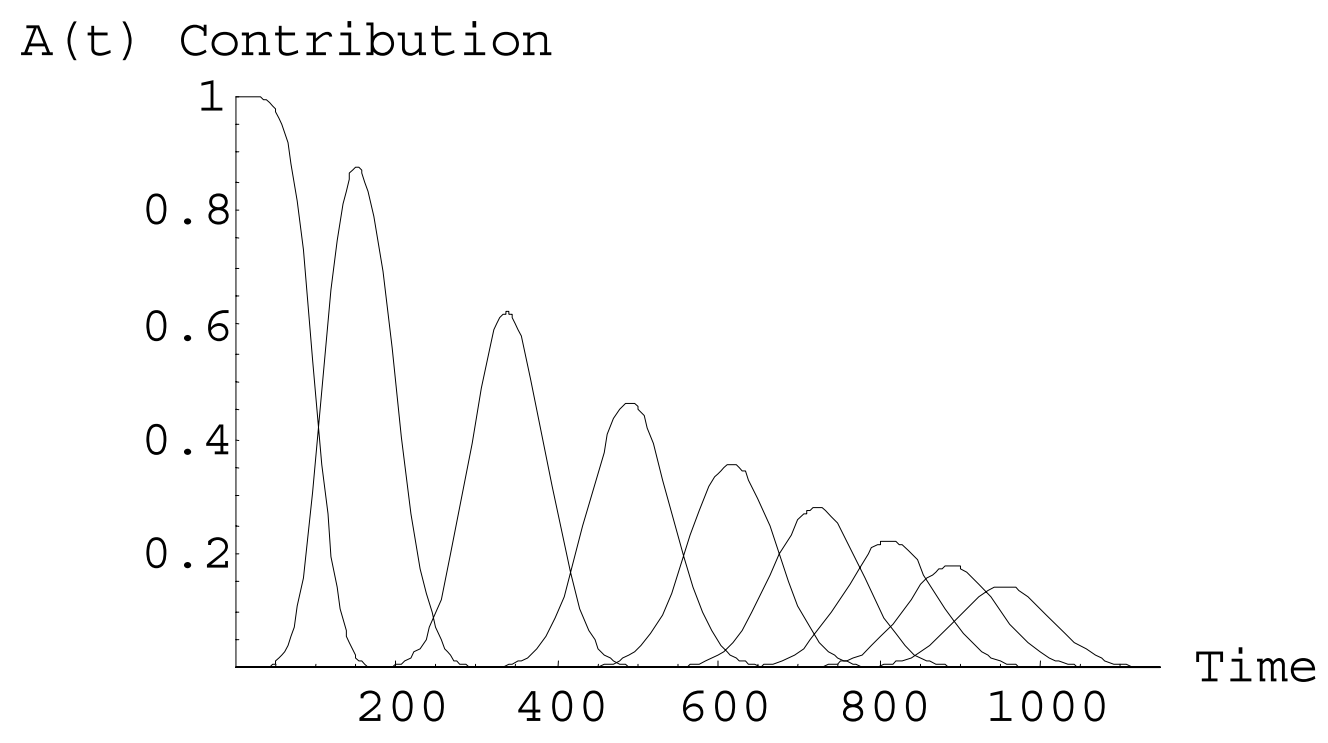

Figure 4.3 Individual Terms for the lower bound on point availability

The contribution that the first several terms in the lower bound makes to the value of the point availability is shown in Figure 4.3. It is important to note that as the process evolves each term makes a smaller contribution to the point availability, but each term has a larger variance than the previous term and therefore makes its contribution over a 
longer interval of time. Truncation limits the number of contributing terms. It becomes apparent from the above graph that when this occurs the lower bound on the availability will drop to 0 when the contribution of the final term becomes negligible. The parallel concept is demonstrated in Figure 4.4 for the upper bound of the point availability.

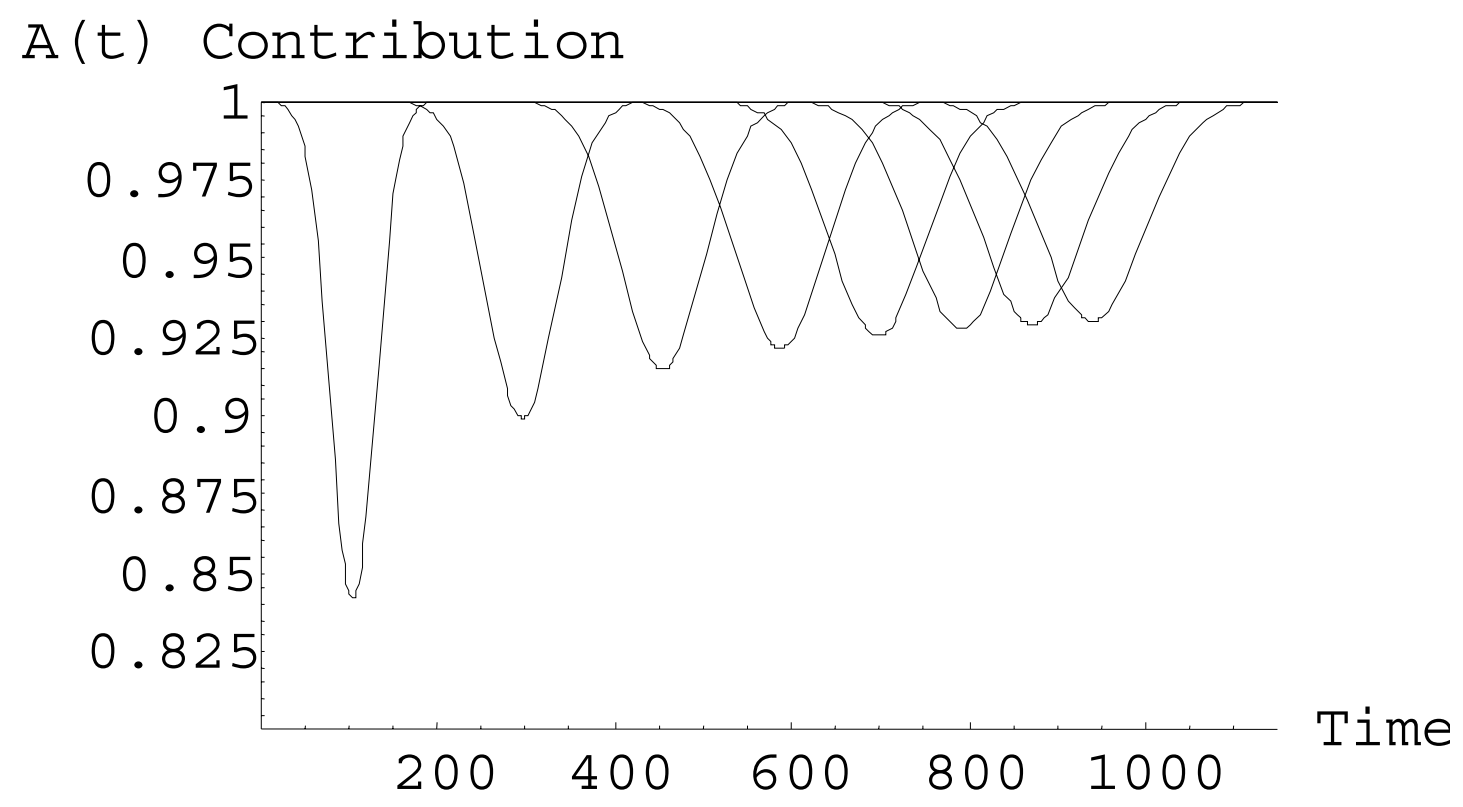

Figure 4.4 Individual terms for the upper bound on point availability

Each term in the sum for the upper bound has a negative contribution to the point availability function, otherwise the same type of behavior is observed. Each term makes a less significant overall contribution but the width of the time interval over which each term makes a contribution is increasing with the cycle number. Truncation limits the number of terms that can make a negative contribution on the point availability. Therefore, the upper bound rises to 1 as the probability mass of the final term becomes negligible. The impact that truncation has on the uptime-based and downtime-based point availability is shown in Figure 4.5. 


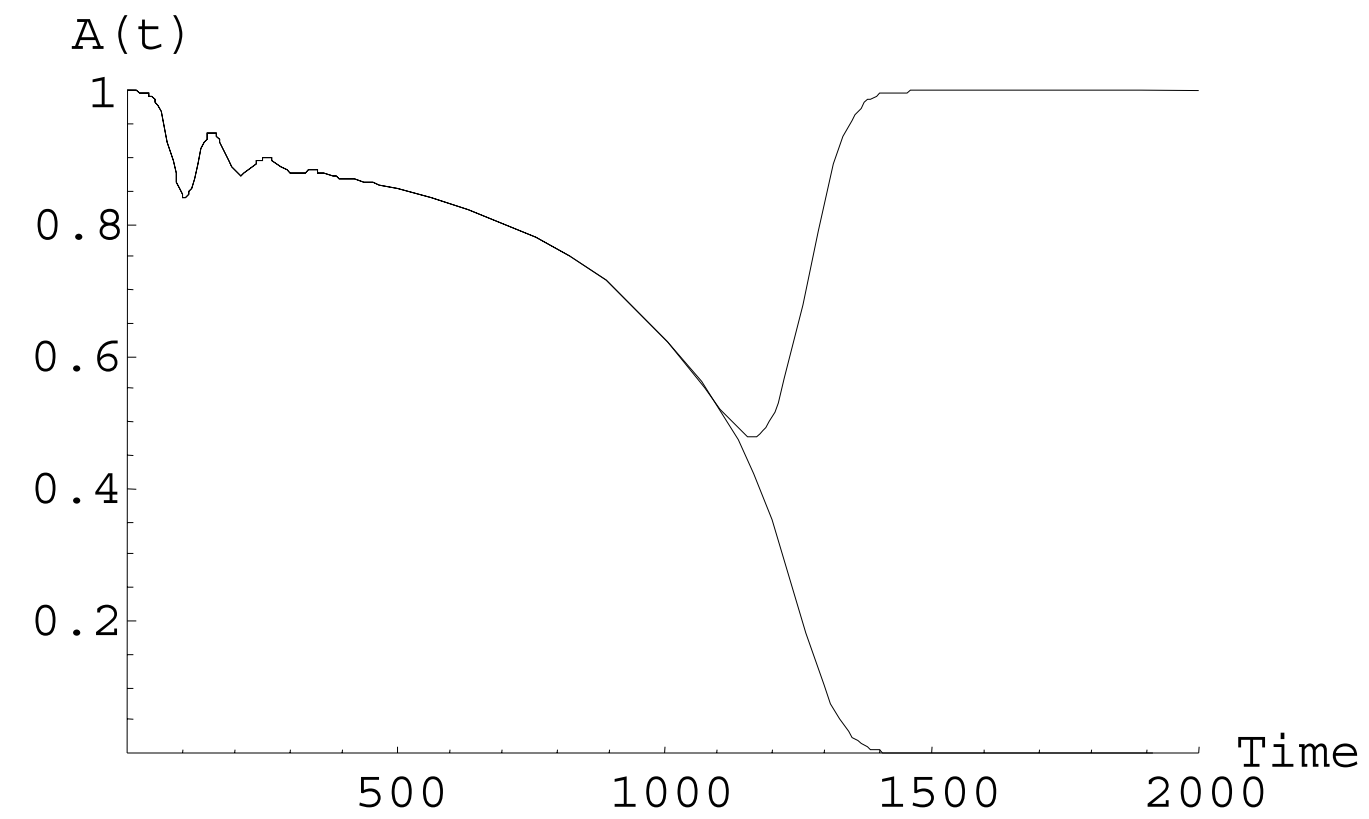

Figure 4.5 Impact of truncation on $A_{H}(t)$ with $c=30$

Increasing the value of $c$ increases the amount of time until the two point availability functions diverge. Decreasing the value of $c$ has the opposite effect. It is clear that the value obtained for the point availability with either function is very accurate up to 1000 time units. After the two functions cease to coincide they diverge rather quickly giving little indication of the location of the true value of the point availability. However, the information gained concerning the interval of time upon which the approximations are accurate is extremely useful. In this situation, it is clear that conclusions can be made with reasonable confidence out to 1000 time units. If it is necessary to forecast farther than 1000 time units, the value of $c$ can be increased.

Since the case of normal operating times and normal repair times is less demanding with respect to computation time, it is possible to evaluate enough terms to drive the upper bound to 0 . When this occurs the point availability is nearly exact since the upper and lower bound coincide until the system has negligible availability. In this manner it is possible to use the upper bound in isolation to obtain an approximation of the point availability to any specified degree of accuracy. This holds true for all situations 
examined although computational demands for the other models make this technique more difficult to implement.

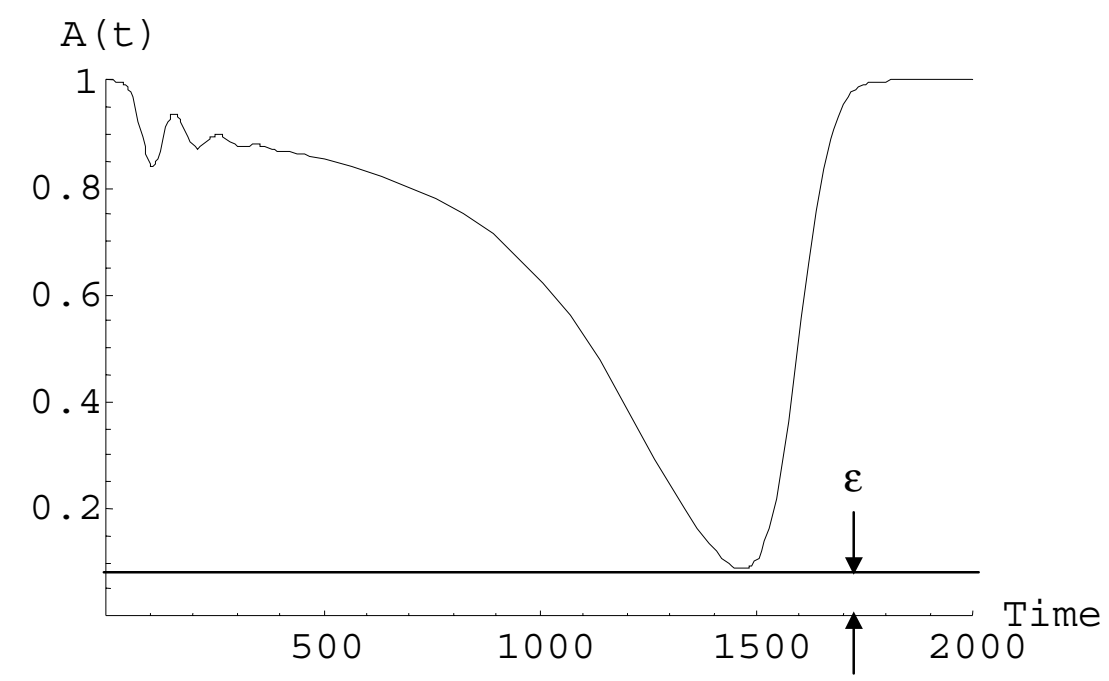

Figure 4.6 Upper bound used to determine error in approximation

In figure 4.6 the upper bound on the point availability is evaluated to a minimum acceptable error value, $\varepsilon$. This corresponds to the maximum error in the point availability approximation for the entire period prior to the time at which $\varepsilon$ is reached. This is true since the lower bound can be no less than 0 , and for any given value of $\mathrm{c}$ the accuracy of the approximation deteriorates with time.

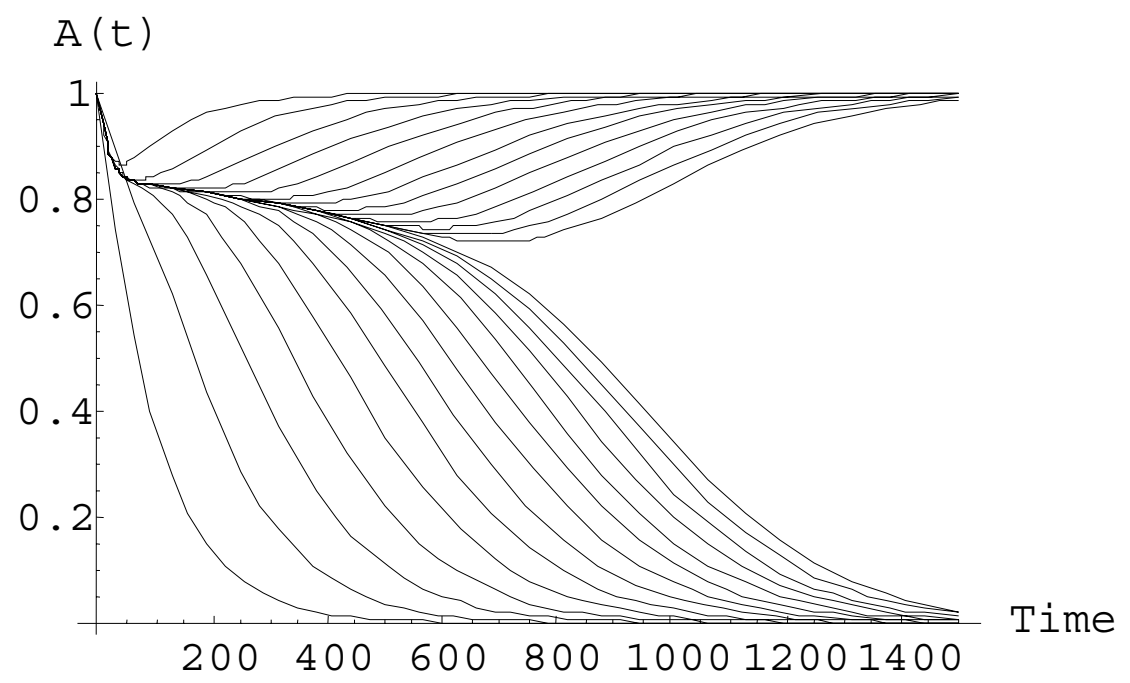

Figure 4.7 Truncation of $A_{H}(t)$ with $(\lambda=0.01, \mu=0.05, c=\{1, \ldots, 13\})$ 
The behavior of the approximating functions as $c$ increases is made clear by Figure 4.7. It is apparent from the above graph that the approximation becomes more accurate as $c$ increases. However, the important information is contained in the two approximate functions with the largest value of $c$ shown in Figure 4.8.

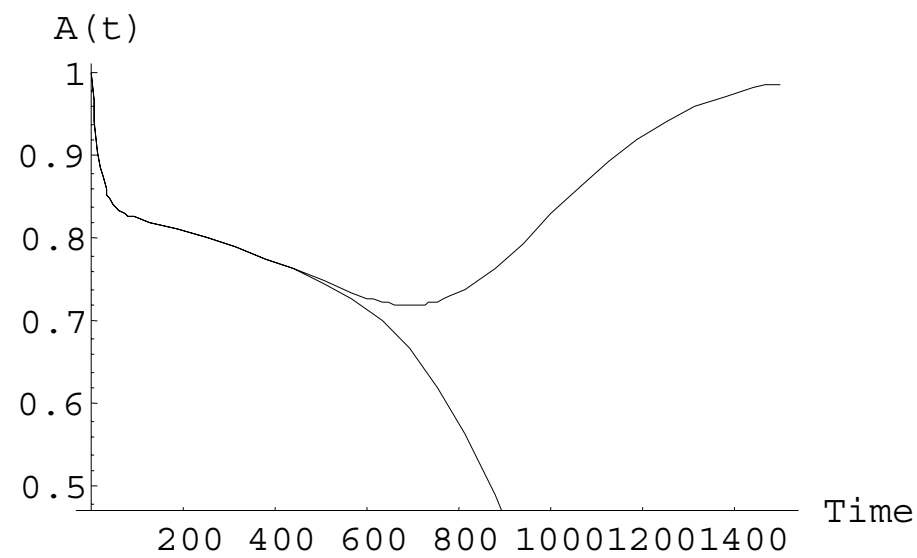

Figure 4.8 Best approximation for $A_{H}(t) \quad(\lambda=0.01, \mu=0.05, c=13)$

These two approximate functions form the tightest bounds on the point availability and give a near exact approximation for the longest period of time.

In addition to the general behavior of the quasi-renewal function and point availability functions, it is necessary to comment on the behavior specific to the various cases analyzed. Specifically, the effect of various deterioration levels and distribution parameters on system behavior is discussed.

\subsection{Exponential Operating Intervals}

Consider intervals of operation that are distributed according to an exponential distribution with rate, $\lambda$. To obtain numerical results in the time domain it is necessary to truncate the infinite sums in the Laplace transform space for the quasi-renewal function and the two point availability functions, equations $3.44,3.45$, and 3.47 respectively. Inverting the terms necessary to approximate the quasi-renewal function takes much less computational time than inverting the terms necessary to approximate either of the point availability functions. 


\subsubsection{Exponential Quasi-Renewal Function}

The first 22 terms of the sum in equation 3.44 were inverted symbolically to the time domain to obtain an approximation of the quasi-renewal function for exponential operating intervals. Describing the behavior of the quasi-renewal function is rather straightforward since the only variables present in the expression are the rate of the exponential distribution, $\lambda$, and the deterioration factor for the operation intervals, $a$. For the purpose of comparison on a common scale, the mean of the first interval of operation is assumed to be 100 time units for all calculations. A graph of three quasi-renewal functions with different deterioration factors is shown in Figure 4.9.

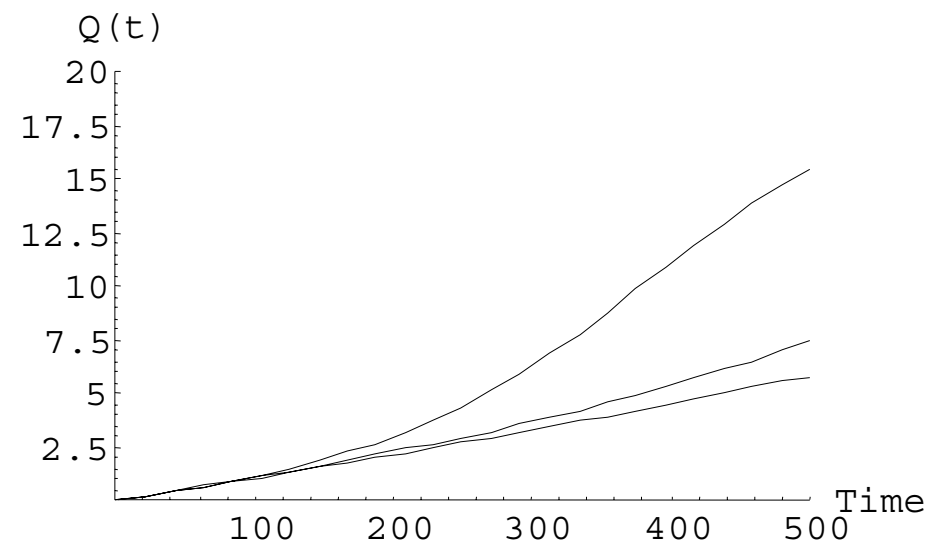

Figure 4.9 Exponential $Q_{F}(t)$ with $(\lambda=0.01, a=\{0.80,0.90,0.95\})$

The behavior of the quasi-renewal function for exponential operating intervals is rather straightforward. For smaller values of the deterioration factor, $a$, the quasi-renewal function increases faster and for larger values of $a$ the quasi-renewal function increases slower.

\subsubsection{Exponential Repair Intervals}

Repair intervals are distributed according to an exponential distribution with rate, $\mu$, and deterioration factor, $b$. The behavior of the point availability is approximated by inverting 12 to 13 terms of the infinite sum expression in the Laplace transform space. Obtaining numerical results from some of the latter terms provided some unexpected 
difficulty related to limitations on computer arithmetic and the machine representation of numbers. This does not affect the qualitative observations made in any way and has a negligible effect on the numerical results that are graphed below. Further discussion is contained later in this chapter. In all cases examined, the mean of the first operating interval is 100 and serves to scale the rest of the analysis. The impact of the length of repair, the rate of deterioration for the operation intervals, and the rate of deterioration for the repair intervals are rather intuitive and is summarized below.

As $a$ decreases the deterioration rate of the system increases and the point availability decreases. This is evident in Figure 4.10. In addition, the point at which the two approximating functions lose accuracy is sooner for smaller values of $a$, causing the point availability bounds to be farther apart at any instance in time than for systems with a larger value of $a$.

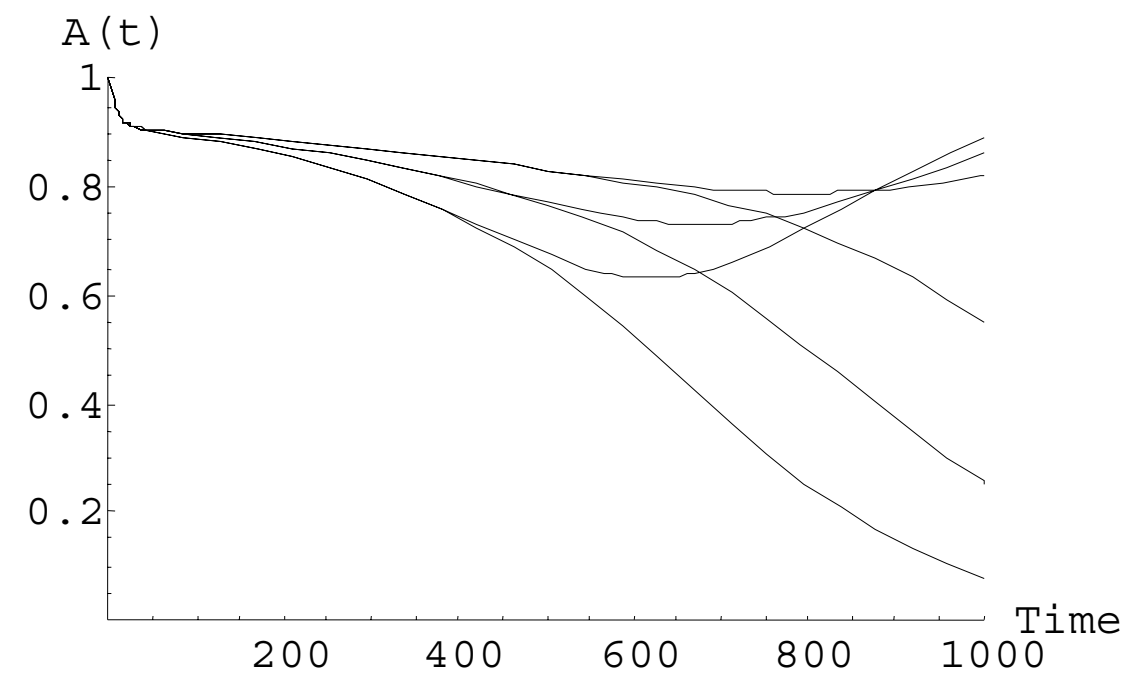

Figure 4.10 Exponential $A_{H}(t)$ with $(\lambda=0.01, a=\{0.85,0.90,0.95\}, \mu=0.10, b=1.1)$

Figure 4.11 shows the impact of varying the mean repair time of the first repair interval. As expected, increasing the mean of the first repair interval causes a reduction in the point availability function. However, the approximation remains accurate for longer periods of time when the mean of the first repair interval is larger. Although the mean of the repair intervals is larger, fewer cycles are completed per unit time and the equipment reaches equivalent levels of deterioration at later points in time. 


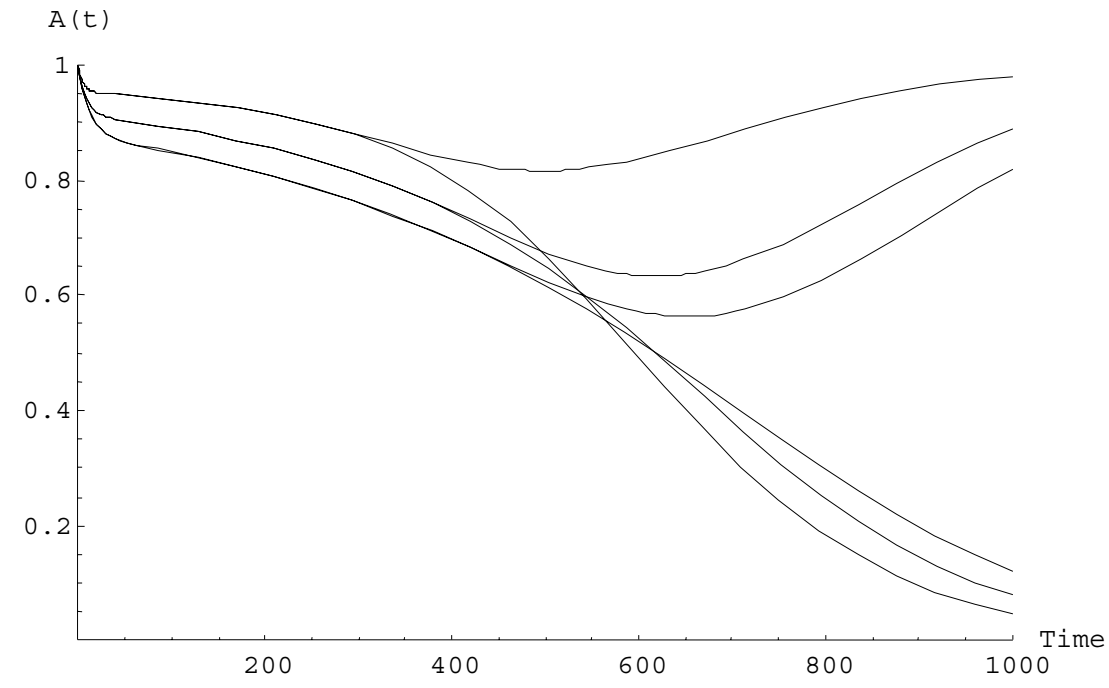

Figure 4.11 Exponential $A_{H}(t)$ with $(\lambda=0.01, a=0.85, \mu=\{1 / 15,1 / 10,1 / 5\}, b=1.1)$

The deterioration of the repair process is also a factor in the behavior of the point availability. Figure 4.12 shows the point availability functions for several different levels of deterioration of the repair process.

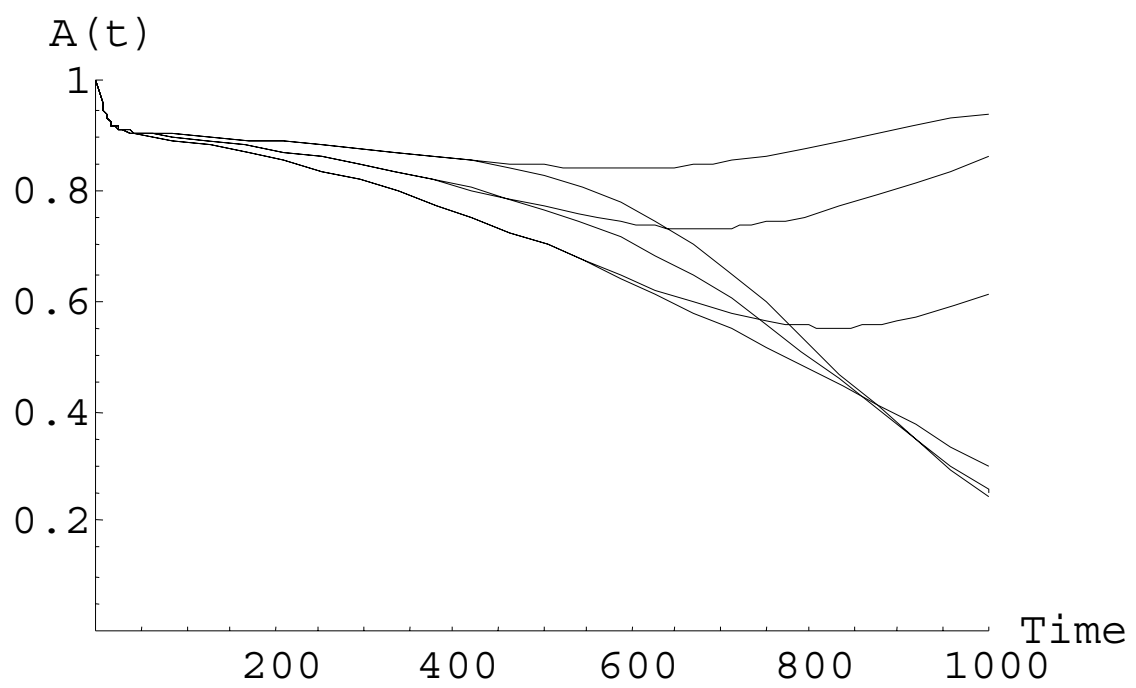

Figure 4.12 Exponential $A_{H}(t)$ with $(\lambda=0.01, a=0.9, \mu=0.10, b=\{1.0,1.1,1.2\})$

As the level of deterioration increases the point availability decreases as expected. Again, the approximation remains accurate longer for a larger level of deterioration since fewer cycles per unit time are completed. 


\subsection{Normally Distibuted Operating Intervals}

Operating intervals are distributed according to a normal distribution with mean, $\mu$, variance, $\sigma^{2}$, and deterioration factor, $a$. In all of the cases examined under the assumption of normally distributed operating intervals, the mean of the first operating interval is 100 time units. As mentioned before, the mean of the first operating interval simply scales the behavior of the quasi-renewal function and the point availability.

\subsubsection{Normal Quasi-Renewal Function}

The normal quasi-renewal function is essentially the expected number of restarts as a function of time when repair time is negligible. Since the system is assumed to deteriorate, the quasi-renewal function is non-decreasing in time. The degree of deterioration has a significant impact on the rate at which the quasi-renewal function increases.

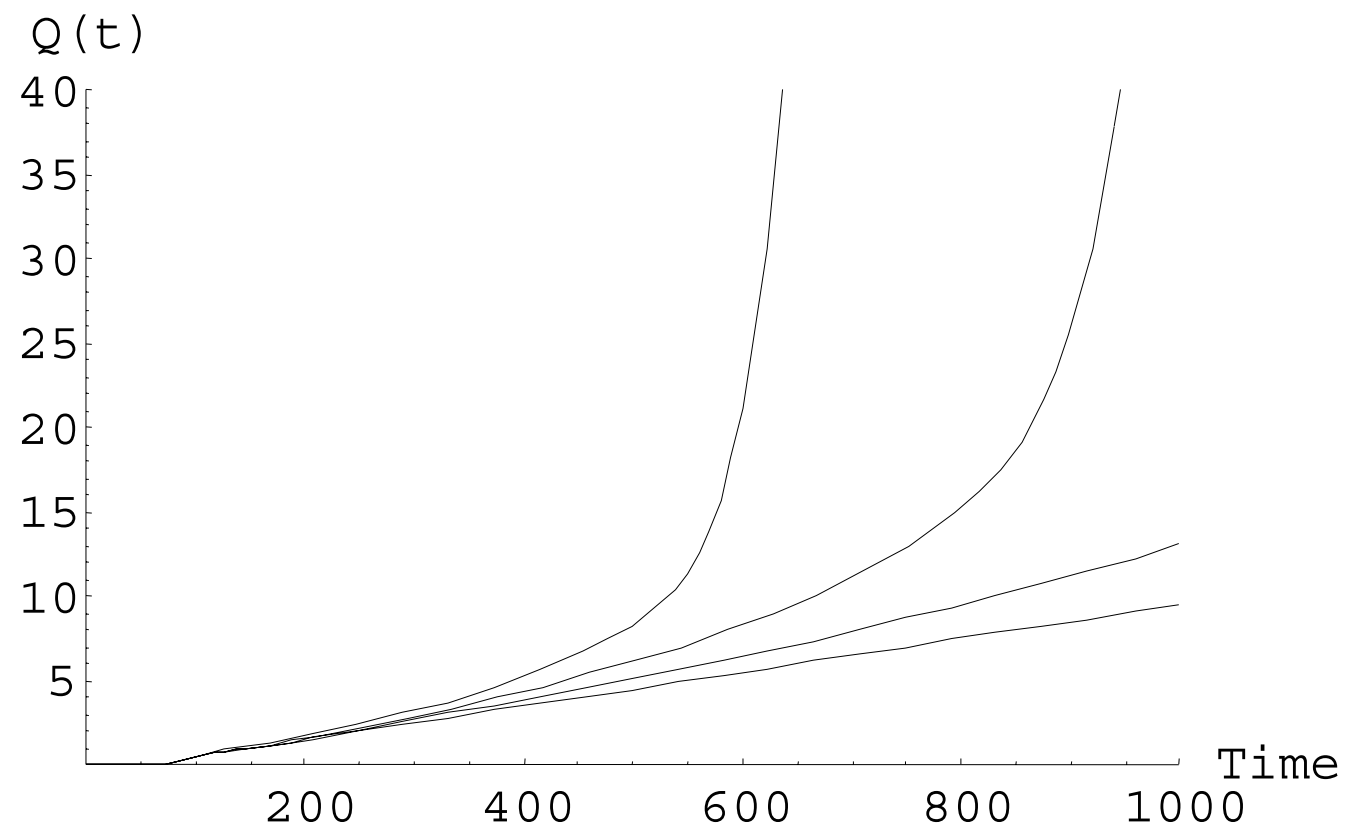

Figure 4.13 Normal $Q_{F}(t)$ with $(\mu=100, \sigma=25, a=\{0.85,0.90,0.95,1.00\})$

This behavior is apparent in Figure 4.13. A smaller value of $a$ indicates a more rapidly deteriorating system and, as shown above, a more rapidly increasing quasi-renewal 
function. The renewal function for a homogeneous process is shown to reinforce the concept that the quasi-renewal process approaches the homogeneous process as the value of $a$ approaches 1 . Another characteristic of the quasi-renewal function is that it appears as though a critical point is reached where the mean of the operation intervals has deteriorated enough so that the expected number of restarts behaves asymptotically. This critical point is reached earlier for smaller values of $a$.

Another factor that affects the behavior of the quasi-renewal function is the ratio between the mean and the variance of the first interval of operation. Each quasi-renewal function in Figure 4.14 corresponds to a quasi-renewal function in Figure 4.13 with the same degree of deterioration. However, the standard deviation of the intervals of operation is 12.5 rather than 25 .

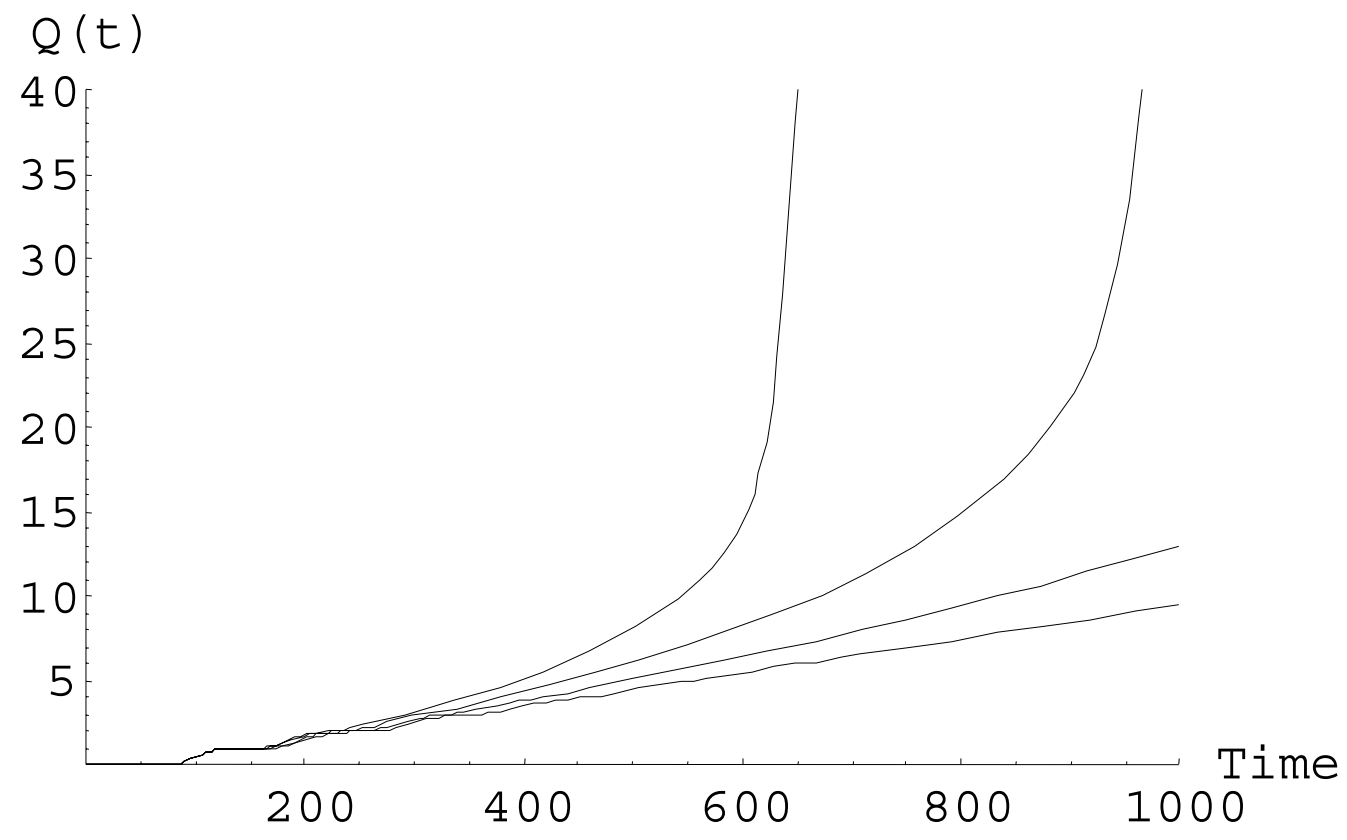

Figure 4.14 Normal $Q_{F}(t)$ with $(\mu=100, \sigma=12.5, a=\{0.85,0.90,0.95,1.00\})$

The overall behavior is the same but it is apparent from Figure 4.15 below that the smaller variance causes the quasi-renewal function to look similar to a step function early in the evolution of the process. In other words, early in the process the quasi-renewal function increases sharply in certain intervals and remains relatively unchanged in others. This effect dissipates as the process evolves. 


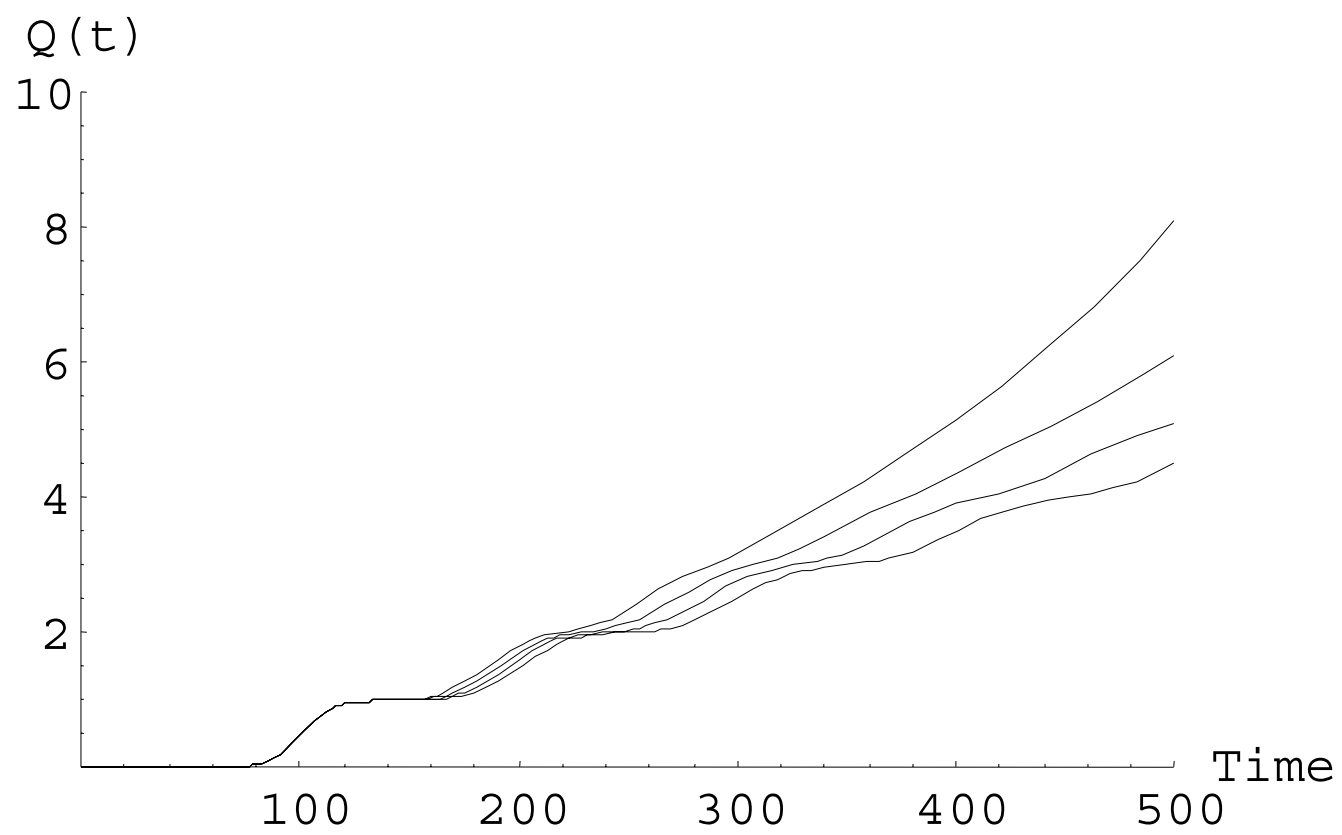

Figure 4.15 Normal $Q_{F}(t)$ with $(\mu=100, \sigma=12.5, a=\{0.85,0.90,0.95,1.00\})$

This is due to the fact that early in the life of the system the first increase in the value of the quasi-renewal function can be attributed to the time at which the first failure occurs. This timing is governed by the distribution on the first operation interval. A small variance causes the timing of the first failure to have a high probability of occurrence in a rather narrow time interval about the mean. The next significant increase in the quasirenewal function is at the most likely interval for the occurrence of the second failure, which is actually the sum of the first two operation intervals. The important fact is that the distribution on the sum of the first two operating intervals has a variance equal to the sum of the variances of the individual operating intervals. This causes the distribution on the time of the second failure to have a significantly larger variance than the distribution on the time of the first failure. This increase in variance propagates over time and causes the quasi-renewal function to show a more uniform behavior rapidly. It should also be noted that this affect dissipates faster for smaller values of $a$, since a small value of $a$ increases the opportunity for a large number of restarts relatively early. 
Figure 4.16 shows the effect that the variance has early in the process holding all other variables constant. As mentioned above, the process with the smaller variance will look similar to a step function longer than a process with a large variance.

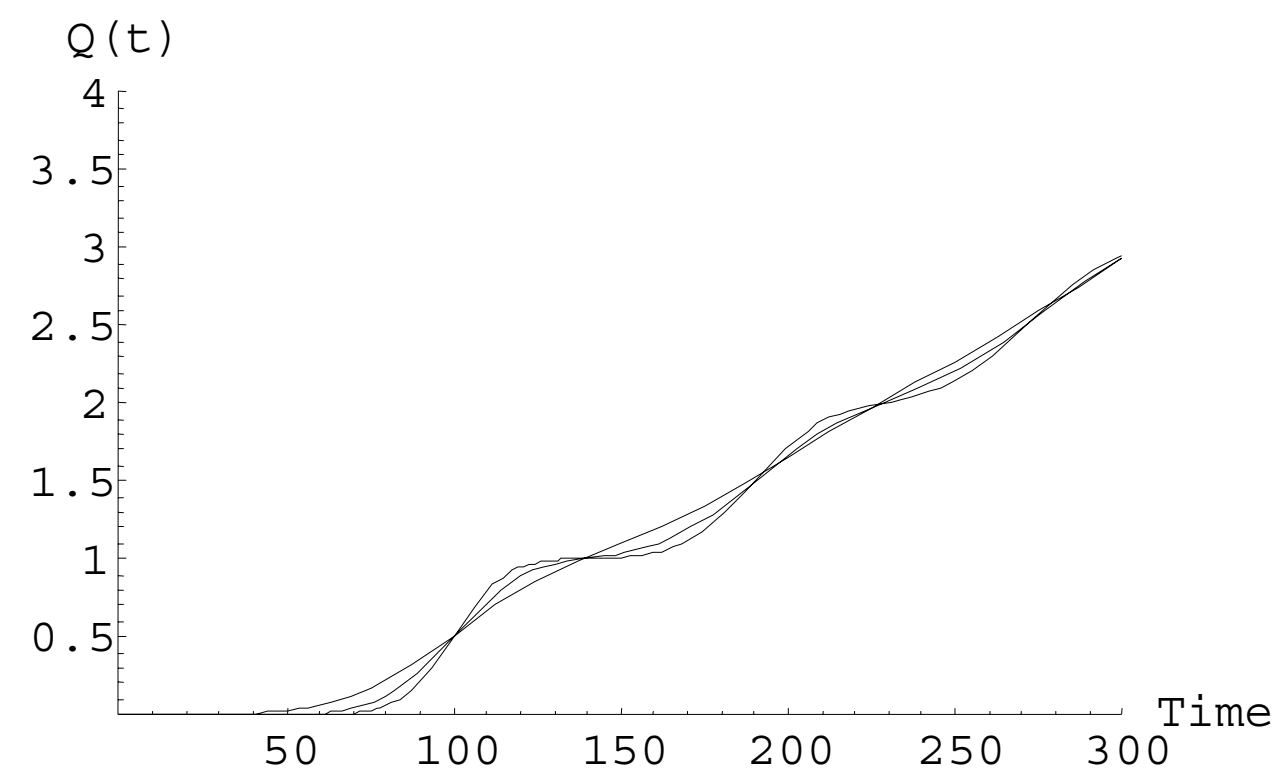

Figure 4.16 Normal $Q_{F}(t)$ with $(\mu=100, \sigma=\{12.5,50 / 3,25\}, a=0.90)$

\subsubsection{Normally Distributed Repair Intervals}

Repair intervals are distributed according to a normal distribution with mean, $\lambda$, variance, $\rho^{2}$, and deterioration factor, $b$. The graph below is representative of the availability function for a quasi-renewal process with normally distributed repair times.

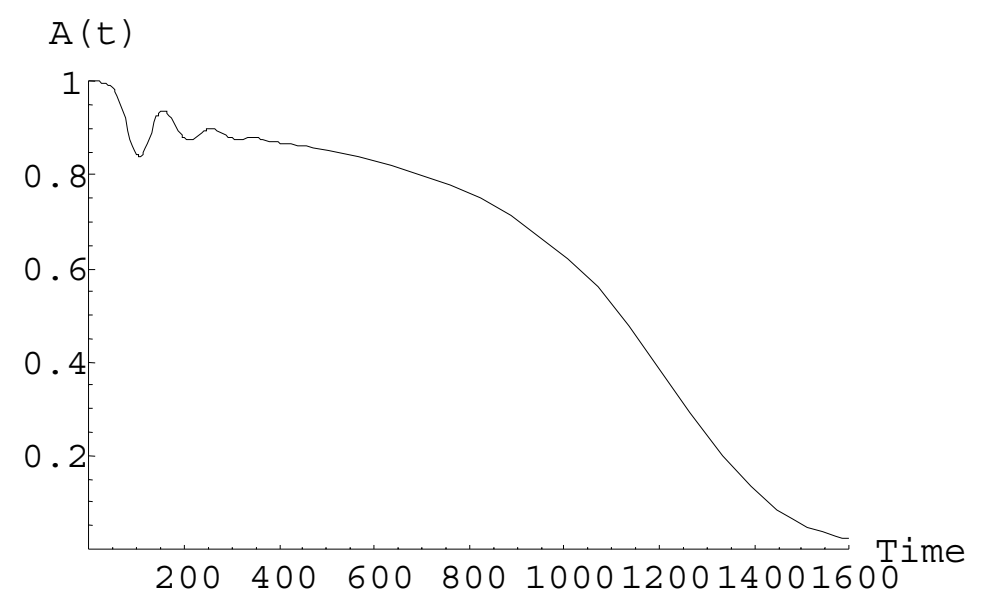

Figure 4.17 Normal $Q_{F}(t)$ with $(\mu=100, \sigma=25, a=0.90, \lambda=10, \rho=2.5, b=1.0)$ 
In the beginning there is a period of fluctuation as the most likely interval for the first failure causes a decrease in the availability and then the most likely interval for the completion of the first repair causes a subsequent increase. As the process evolves this fluctuation dissipates and the availability goes to 0 as the length of the intervals of operation deteriorates. This fluctuation is more pronounced for operation intervals with a smaller variance, which is shown in Figure 4.18.

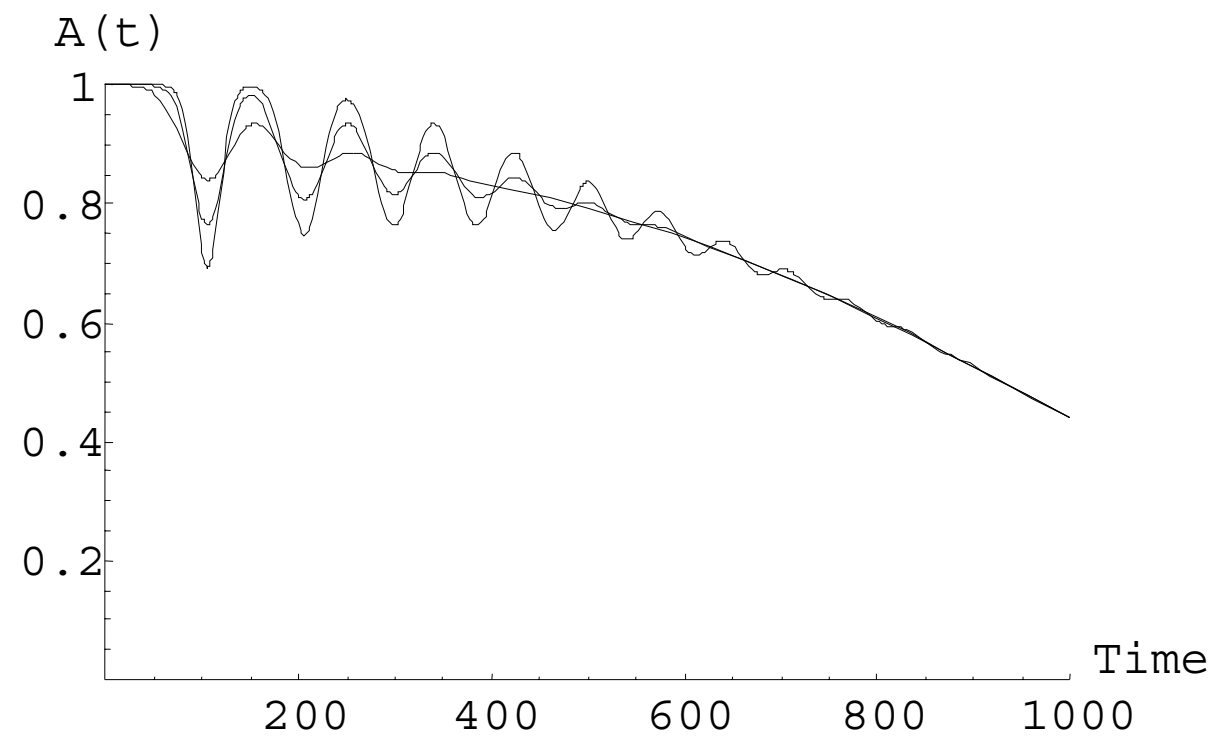

Figure 4.18 Normal $A_{H}(t)$ with

$$
(\mu=100, \sigma=\{12.5,50 / 3,25\}, a=0.90, \lambda=10, \rho=2.5, b=1.1)
$$

Three different values for the standard deviation of the first interval of operation are used while holding all other variables constant to graph the quasi-renewal functions above. The quasi-renewal function with a standard deviation of 12.5 has the largest fluctuation that takes the longest to dissipate. The quasi-renewal function with a standard deviation of 25 fluctuates mildly in the beginning and then is relatively smooth after 350 time units.

The deterioration factor, $a$, has a significant impact on the rate at which the point availability decreases and the time it takes for the system to become impractical to operate without a replacement or overhaul. Three point availability functions are graphed 
in Figure 4.19, each with a different level of deterioration for the operation interval and the remaining variables are identical between functions.

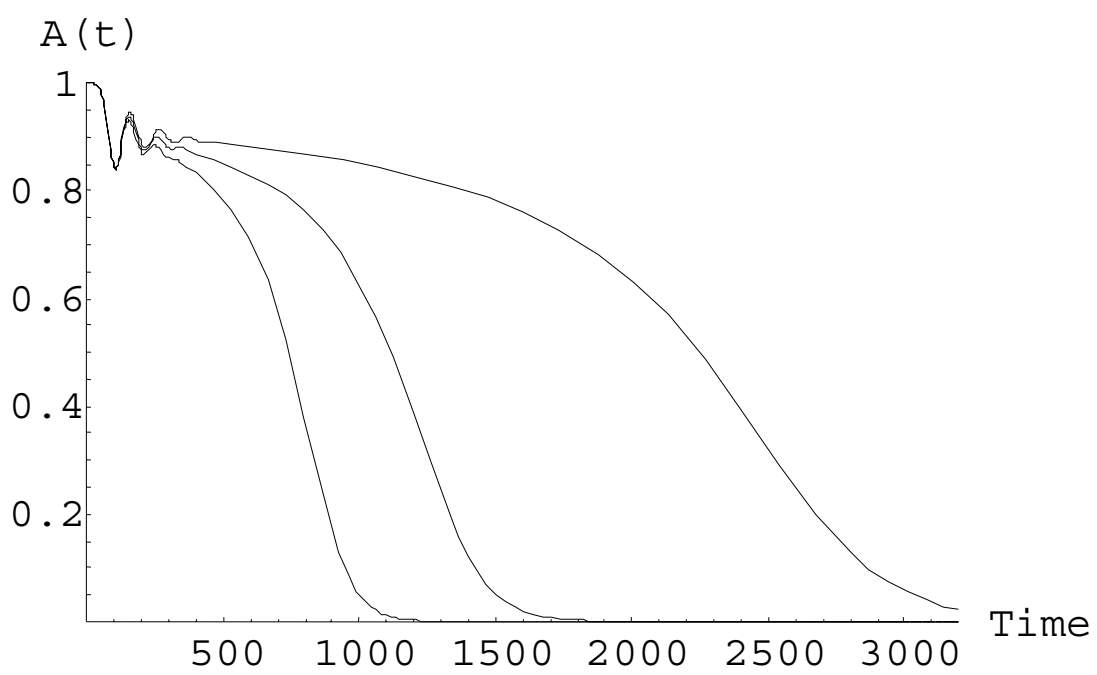

Figure 4.19 Normal $A_{H}(t)$ with

$$
(\mu=100, \sigma=25, a=\{0.85,0.90,0.95\}, \lambda=10, \rho=2.5, b=1.0)
$$

Similarly, the level of deterioration in the repair process, $b$, has a significant impact on the behavior of the availability function. Figure 4.20 shows three different point availability functions that have different levels of repair deterioration while holding all other variables constant.

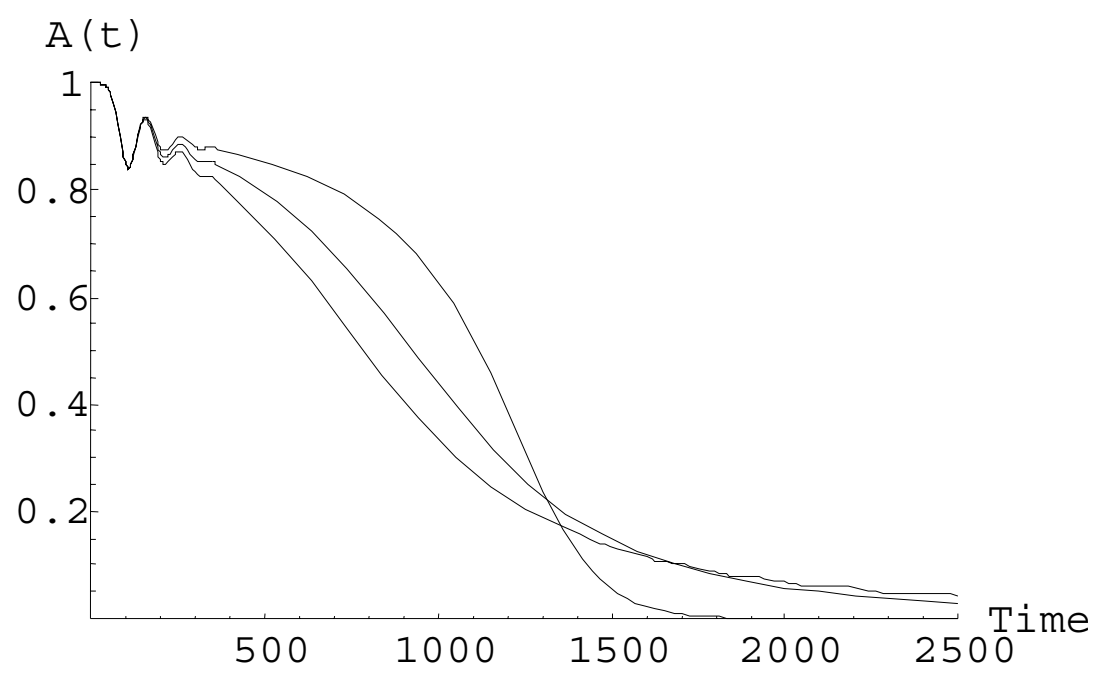

Figure 4.20 Normal $A_{H}(t)$ with

$$
(\mu=100, \sigma=25, a=0.90, \lambda=10, \rho=2.50, b=\{1.0,1.1,1.2\})
$$


The point availability function that has a negligible availability at 2000 time units corresponds to the iid repair case, $b=1.0$. The values for the iid point availability function are the highest early in the evolution of the process, but then become the lowest at about 1350 time units. Because repairs are quicker on average for the iid repair case the proportion of time that the system is being repaired is smaller than for the other two deteriorating repair cases. However, since the repairs occur more quickly, the time it takes to reach the $n^{\text {th }}$ interval of operation in the iid repair case is smaller than the other two cases. This means that the iid repair process will reach the stochastically smaller operation intervals faster than the deteriorating repair processes. At 1350 time units the fact that on average the iid repair process reaches operation intervals that are stochastically smaller at a quicker pace outweighs the fact that the repair intervals are not increasing. The opposite is true for the quasi-renewal process with a larger degree of repair deterioration, $b=1.2$. In the beginning the repair takes longer on average and the system is not as available. As time passes, the fact that repairs take longer in the beginning implies that on average at any time in the future the process will finish a smaller number of cycles and have stochastically larger operating intervals. However, there is little practical advantage to having a process with the highest availability when that availability is near 0.20 .

In addition to the rate of deterioration of repair, the mean value of the first repair interval relative to the mean value of the first operation interval is important in determining the behavior of the point availability function. Figure 4.21 shows the point availability function for three cases in which the mean repair times are different while the remaining variables are identical. Behavior similar to that described above is evident. The case with the smallest mean repair, 5 time units, has the highest availability at first. However, smaller mean repair times cause quicker cycle completions and stochastically smaller operation intervals at earlier points in time than cases with a larger mean repair time. This causes the case with the smallest mean repair time to have the highest point availability early in the evolution of the process and end up with the lowest point availability in the long run. 


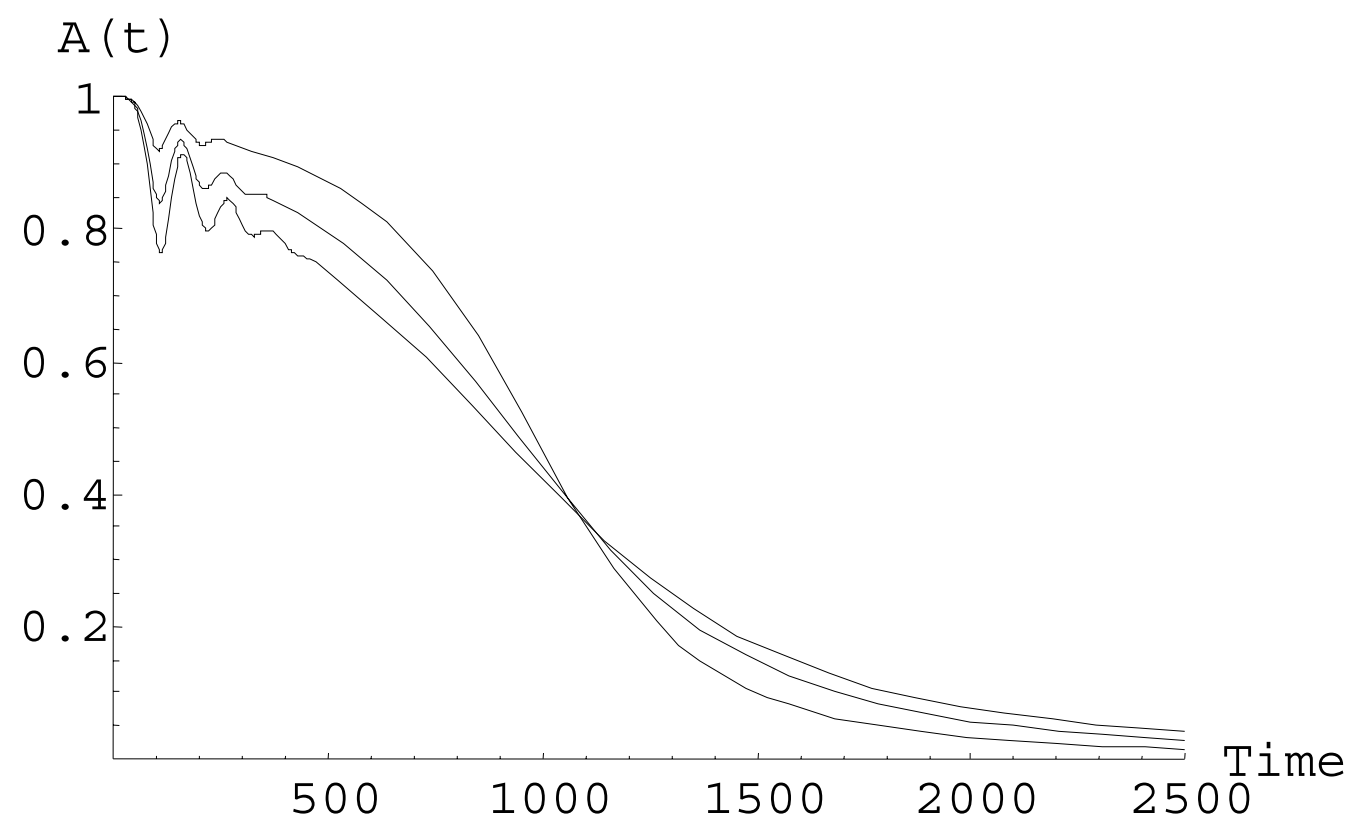

Figure 4.21 Normal $A_{H}(t)$ with

$$
(\mu=100, \sigma=25, a=0.90, \lambda=\{5,10,15\}, \rho=2.5, b=1.1)
$$

A final consideration is the effect of the variation of the first repair interval on the point availability. Since the average length of the repair intervals is relatively small when compared to the average length of the operation intervals at the beginning of the process, the variation of the repair distribution has a negligible impact on the point availability function. Three point availability functions that have different repair variances are graphed in Figure 4.22. The point availability functions practically coincide, indicating that the variation in the repair distribution is relatively significant. The length of the repair intervals does become greater than the length of the operation intervals late in the evolution of the process. However, this occurs when the frequency of repair drives the availability of the system to 0 . In practice this would occur toward the end of the useful life of an ordinary system and replacement would most likely precede that event. 


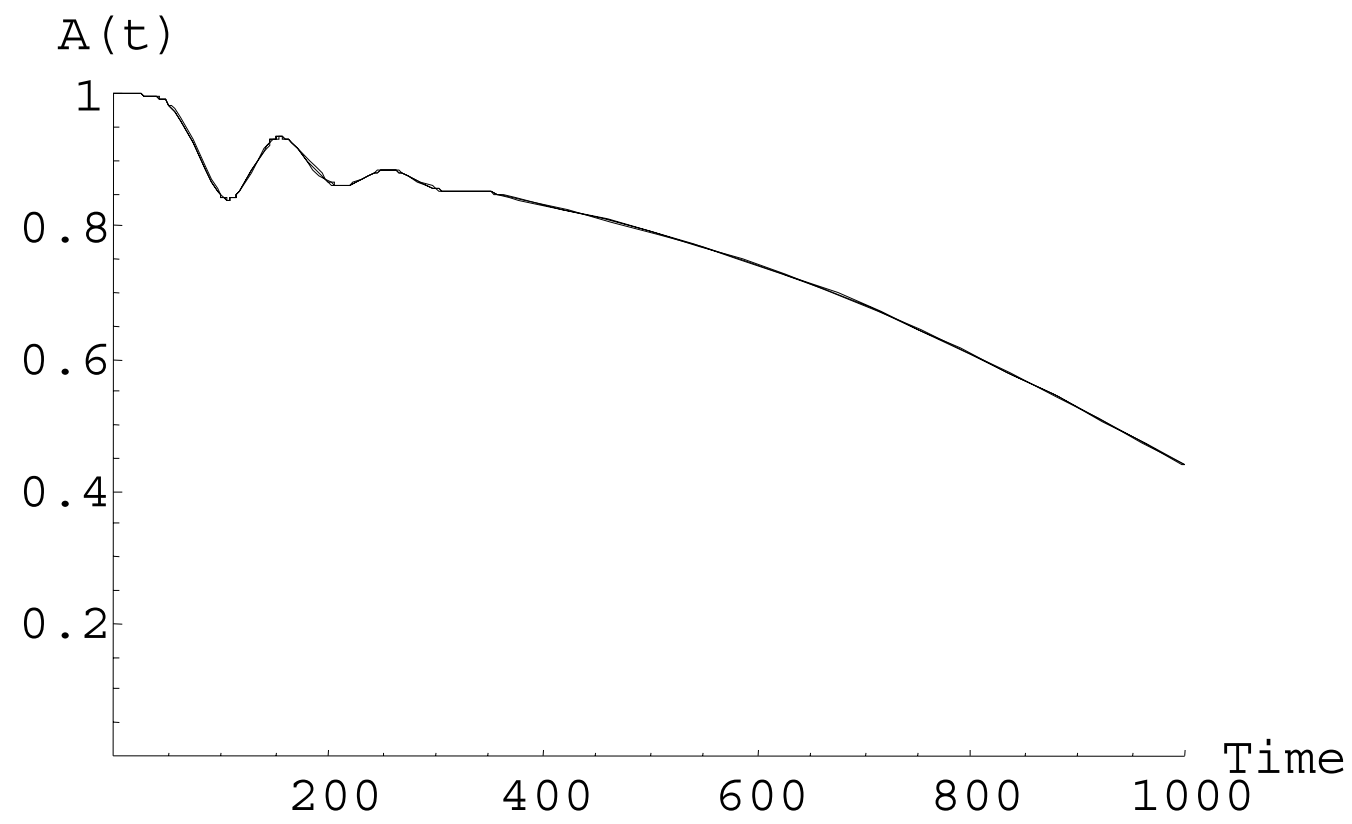

Figure 4.22 Normal $A_{H}(t)$ with

$$
(\mu=100, \sigma=25, a=0.90, \lambda=10, \rho=\{2.50,3.33,5\}, b=1.1)
$$

\subsubsection{Exponential Repair Intervals}

The analysis of the case in which operation intervals follow a normal distribution and repair intervals follow an exponential distribution is not provided. This analysis requires the capability to invert Laplace transforms of the form $\frac{e^{m s-q s^{2}}}{(\lambda+s)^{n}}$ where $m$ and $q$ are real numbers, $\lambda$ is the real number corresponding to rate of the exponential repair time, and $n$ is an integer that corresponds to the term being evaluated. Mathematica is incapable of inverting such an expression either symbolically or numerically and standard Laplace transform tables do not contain a reference to this form. 


\subsection{Gamma Operating Intervals}

The gamma quasi-renewal function and point availability behave in a manner very similar to that of the corresponding exponential functions. This is not surprising since the sum of iid exponential random variables follows a gamma distribution and the exponential distribution is a special case of the gamma distribution. Operating intervals are distributed with a gamma distribution with shape parameter, $k$, scale parameter, $\lambda$, and deterioration factor, $a$. The quasi-renewal function is examined under different values of $k$ and $a$ in the next section. Subsequently, the behavior of the point availability functions is examined under various conditions. It is important to recognize that the gamma operating intervals have mean $\frac{k}{\lambda}$ and variance $\frac{k}{\lambda^{2}}$. This relationship between the shape parameter and the scale parameter is used to scale the functions for comparison.

\subsubsection{Gamma Quasi-Renewal Function}

The quasi-renewal function for gamma operating intervals is approximated with the first 20 terms in the infinite sum for $k=2$. Figure 4.23 shows the quasi-renewal function with a mean of 100 for the first interval of operation and three levels of the deterioration factor. As in all other cases examined, the quasi-renewal function increases faster for smaller values of $a$ which corresponds to a quicker system deterioration. The expected number of restarts reaches the maximum of 20 earlier for smaller values of the deterioration factor. Therefore, the accuracy of the approximation based on the first 20 terms for $a=0.80$ is not adequate for as long as the approximation for $a=0.90$. 


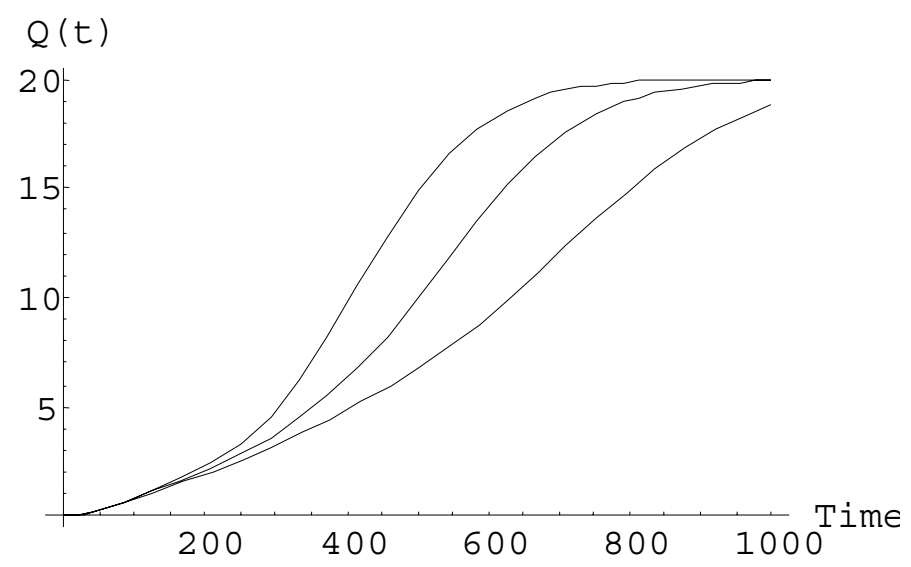

Figure 4.23 Gamma $Q_{F}(t)$ with $(k=2, \lambda=0.02, a=\{0.80,0.85,0.90\})$

The quasi-renewal function is shown in Figure 4.24 under the same deterioration levels, but with a different value of the mean of the first operation interval. Since the mean of the first operation interval in this case is 66.67 the expected number of renewals at any point in time is larger than in Figure 4.23. Since the limit of the quasi-renewal function based on 20 terms is reached relatively quickly, this approximation is adequate for a shorter amount of time than the approximation based on 20 terms above. This is because a larger number of cycles are completed in a shorter amount of time with a smaller mean for the first operation interval.

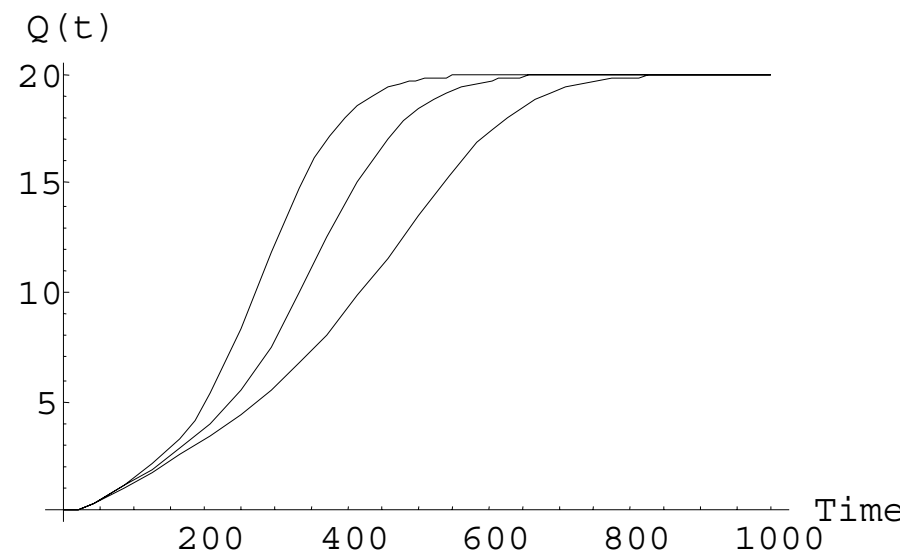

Figure 4.24 Gamma $Q_{F}(t)$ with $(k=2, \lambda=0.03, a=\{0.80,0.85,0.90\})$

The quasi-renewal function for gamma operating intervals with $k=3$ and mean for the first operating interval of 100 is shown in Figure 4.25. Note that the maximum value of the quasi-renewal function is 18 since the first 18 terms were used in this approximation. 


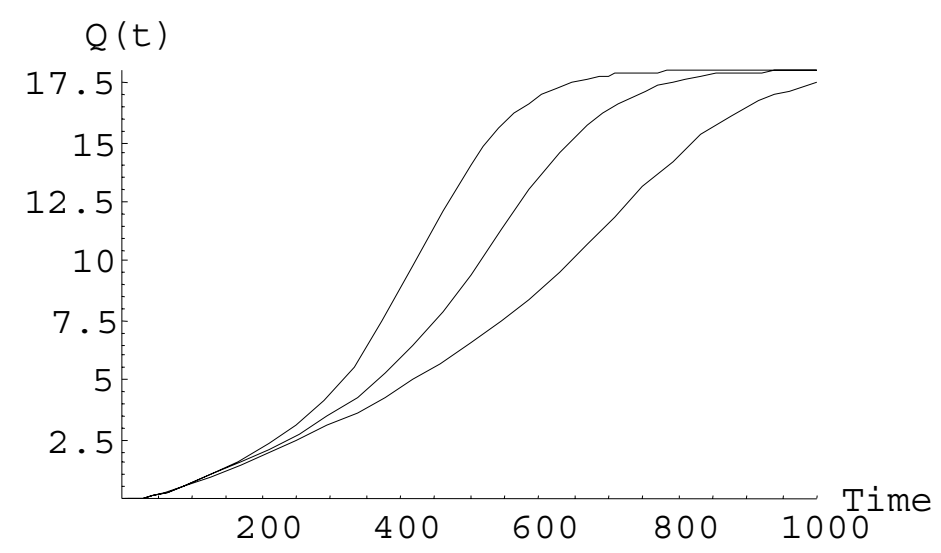

Figure 4.25 Gamma $Q_{F}(t)$ with $(k=3, \lambda=0.03, a=\{0.80,0.85,0.90\})$

To compare the gamma quasi-renewal function with $k=2$ to the gamma quasi-renewal function with $k=3$ the approximation for both functions is made with 18 terms. The value of the mean of the first operation interval for both quasi-renewal functions is the same. Figure 4.26 shows the quasi-renewal function with the same three levels of deterioration for both $k=2$ and $k=3$.

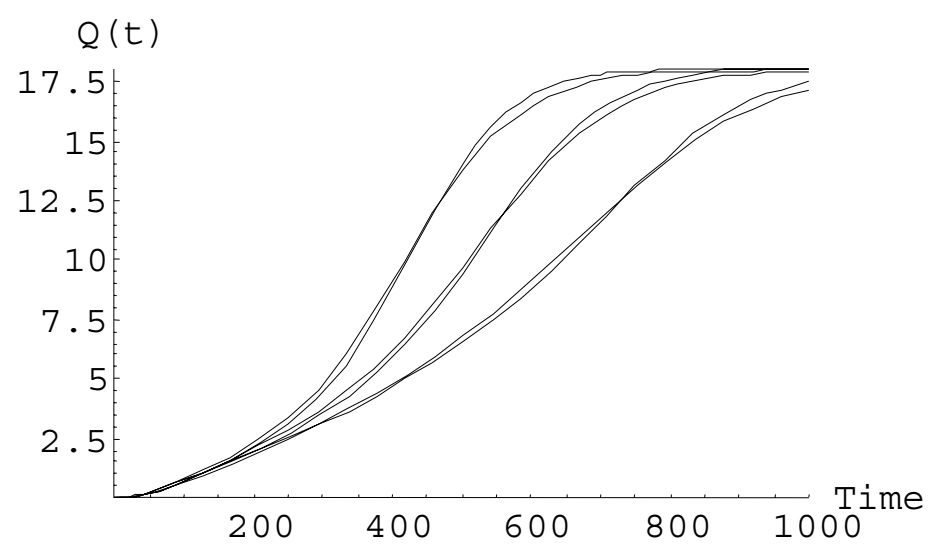

Figure 4.26 Gamma $Q_{F}(t)$ with $(k=\{2,3\}, \lambda=0.03, a=\{0.80,0.85,0.90\})$

This shows that there is little difference between the quasi-renewal function approximations with $k=2$ and $k=3$. However, in cases with a larger difference between the values of $\mathrm{k}$ the difference may become more pronounced. The quasi-renewal function for $k=2$ is larger than the quasi-renewal function for $k=3$ since its probability mass is skewed more toward smaller values of time. 


\subsubsection{Exponential repair intervals}

Repair intervals follow an exponential distribution where $\mu$ is the rate of the first repair interval and $b$ is the deterioration factor for repair. The impact of the mean of the first repair interval and the deterioration factors for operation and repair on the point availability function for gamma operation intervals and exponential repair intervals is examined. The mean of the first gamma operational interval is 100 time units for consistency. Figure 4.27 shows the bounds on the point availability for three different degrees of repair deterioration.

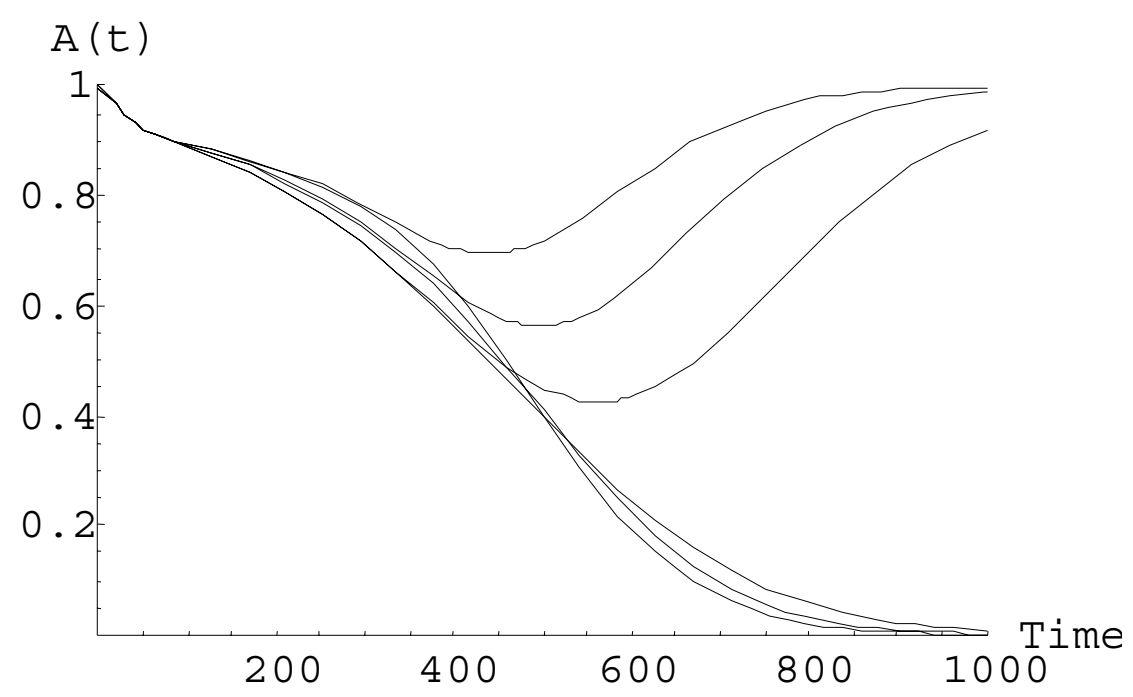

Figure 4.27 Gamma $A_{H}(t)$ with

$$
(k=2, \lambda=1 / 50, a=0.85, r=1, \mu=1 / 10, b=\{1.1,1.2,1.3\})
$$

The point availability remains higher for less severe deterioration conditions in the region where the upper and lower bounds are very tight. The upper bound approximations maintain this relationship for the remainder of the time, however the lower bounds do not. The upper bound corresponding to a smaller deterioration factor starts with larger availability values but after 500 time units the upper bound has the lesser of the three point availability values. This behavior is described earlier. It should be noted that the upper bound is based on the downtime-based point availability function, which is more sensitive to changes in the distribution of the repair intervals. A situation in which the lower bound maintains the expected relationship is shown below. 
A mirror image of the behavior above can be observed in Figure 4.28. This is a graph of the point availability function with three different levels of deterioration in the operating intervals. This causes the point availability to remain higher for the slower deterioration levels in the region where the upper and lower bounds are tight. The lower bound maintains this relationship, however the upper bounds exchange relative positions as the process evolves. This is due to the fact that the lower bound is given by the uptime-based point availability approximation that is more dependent upon the distribution of the operating intervals than the downtime-based point availability function.

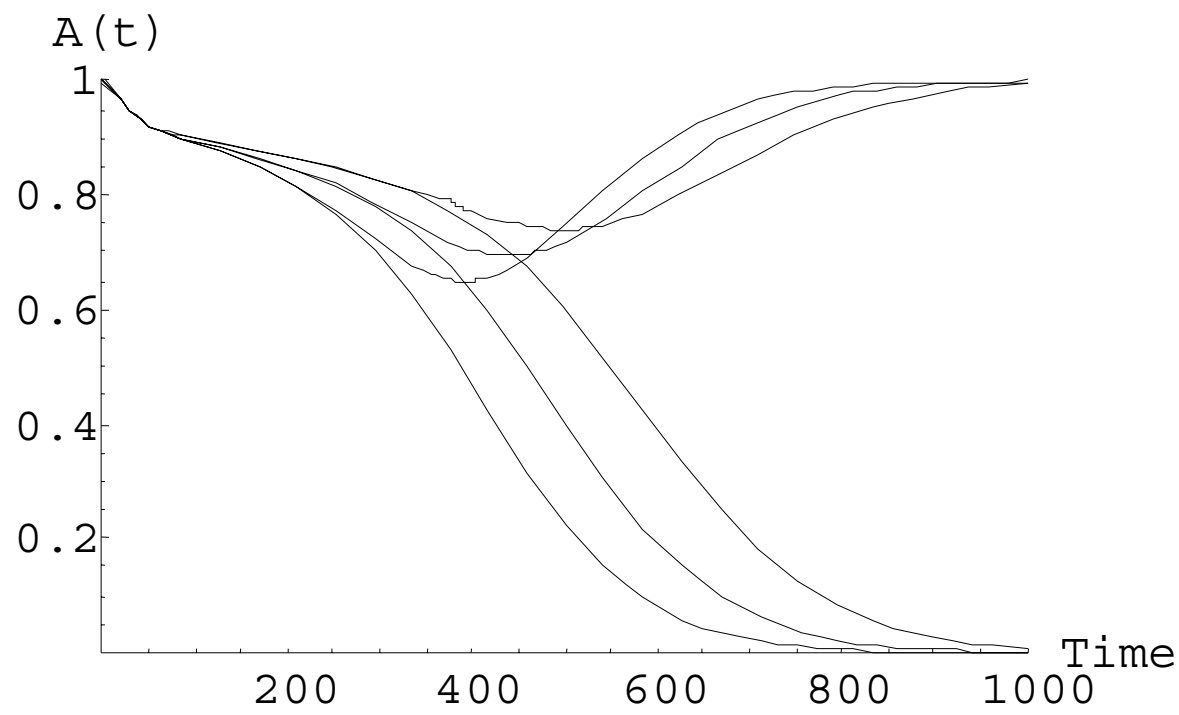

Figure 4.28 Gamma $A_{H}(t)$ with

$$
(k=2, \lambda=1 / 50, a=\{0.75,0.80,0.85\}, r=1, \mu=1 / 10, b=1.1)
$$

Finally, variation of the mean of the first operating interval is studied in Figure 4.28. The point availability is larger for smaller mean repair times while the upper and lower bounds are tight. This relationship is maintained in the upper bound since it is the downtime-based availability function and depends more heavily on the distribution of the repair intervals. 


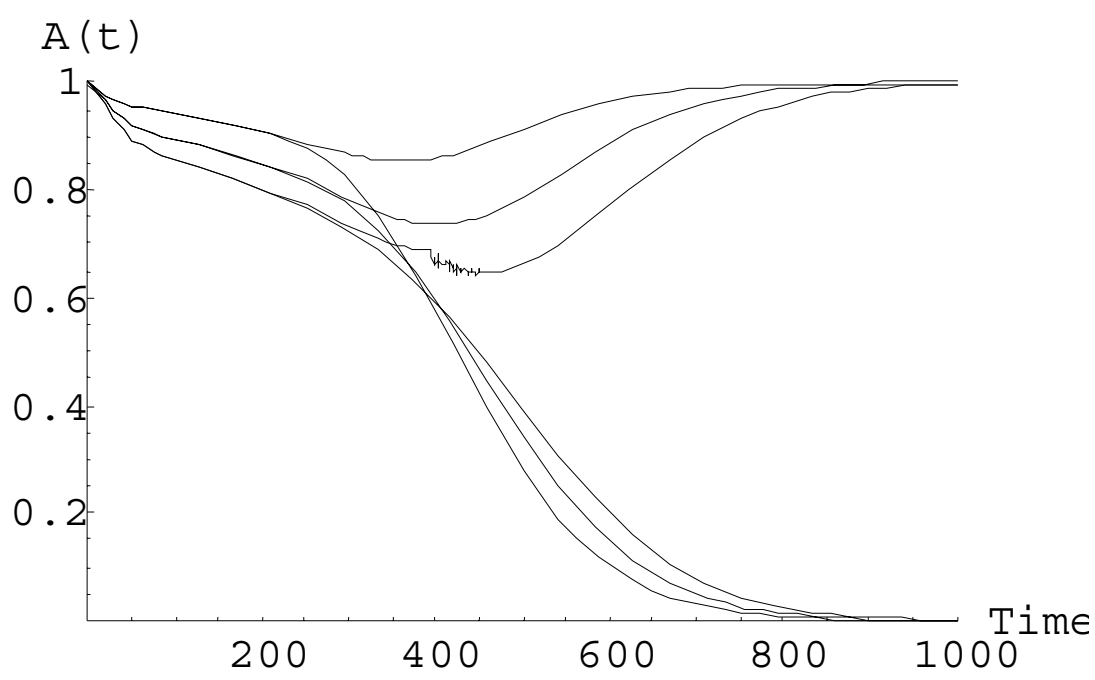

Figure 4.29 Gamma $A_{H}(t)$ with

$$
(k=2, \lambda=1 / 50, a=0.85, r=1, \mu=\{1 / 5,1 / 10,1 / 15\}, b=1.1)
$$

\subsubsection{Gamma repair intervals}

The point availability behavior of a quasi-renewal process with gamma distributed operation intervals and gamma distributed repair intervals is examined below. Operation intervals are distributed according to a gamma distribution with shape parameter, $k$, scale parameter, $\lambda$, and deterioration factor, $a$. Repair intervals are distributed according to a gamma distribution with shape parameter, $r$, scale parameter, $\mu$, and deterioration factor, $b$. Computational limitations allowed only the case where $r=k=2$.

The deterioration factor for the operation intervals is varied in Figure 4.30 for the point availability function. Again, a variation in the parameter of the operation intervals causes a distinct change in the lower bound since it is calculated using the uptime-based point availability approximation. The point availability is larger for larger values of the deterioration factor that corresponds to a slower rate of deterioration. 


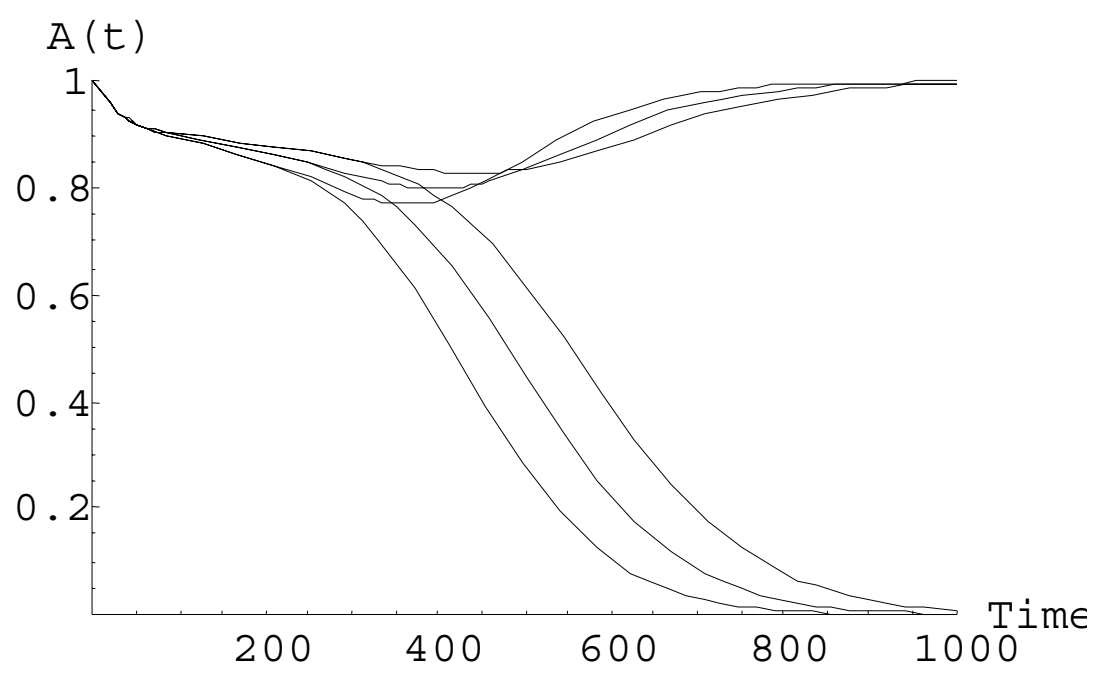

Figure 4.30 Gamma $A_{H}(t)$ with

$$
(k=2, \lambda=2 / 100, a=\{0.80,0.85,0.90\}, r=2, \mu=2 / 10, b=1.1)
$$

The point availability in Figure 4.31 exhibits the corresponding behavior for changes in a repair parameter. Specifically, three different repair deterioration factors are shown below. In this case the upper bound exhibits a distinct increase for smaller values of the repair deterioration factor that correspond to slower deterioration in the repair process. In addition the point availability for smaller values of the repair deterioration is larger in the time interval where the upper and lower bounds are tight.

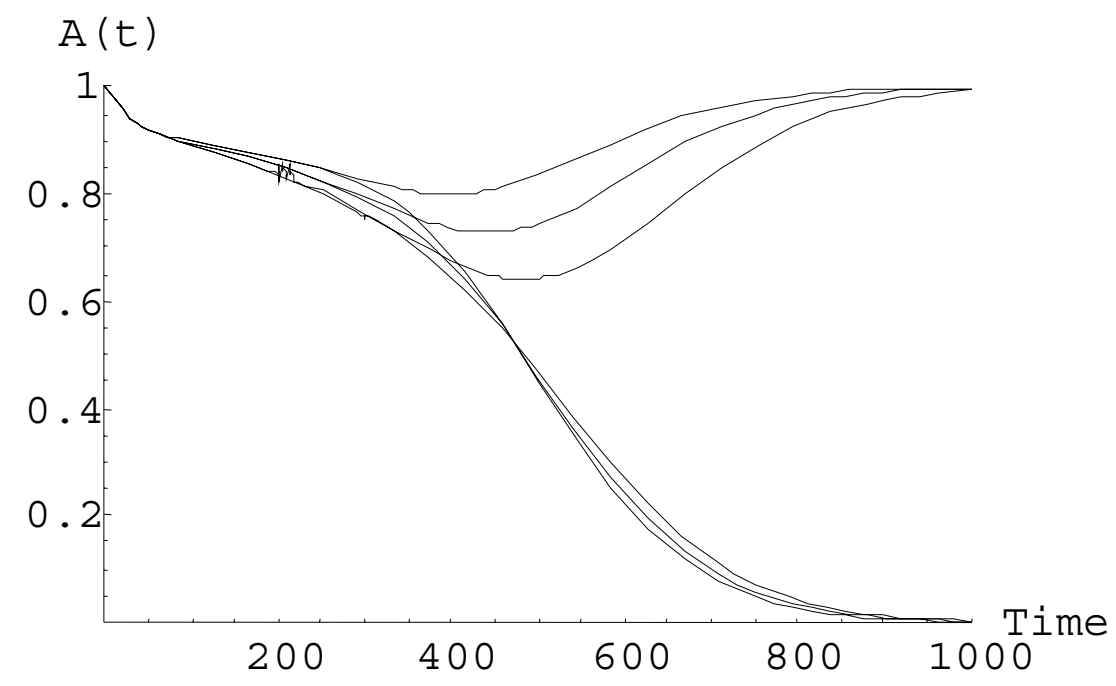

Figure 4.31 Gamma $A_{H}(t)$ with

$$
(k=2, \lambda=2 / 100, a=0.85, r=2, \mu=2 / 10, b=\{1.1,1.2,1.3\})
$$


Finally, the impact of changing the mean of the first operating interval is examined in Figure 4.32. As in other examples, this has an effect that is similar to altering the repair deterioration factor. The point availability is larger for a smaller mean in the first repair interval in the region where the upper and lower bounds are tight.

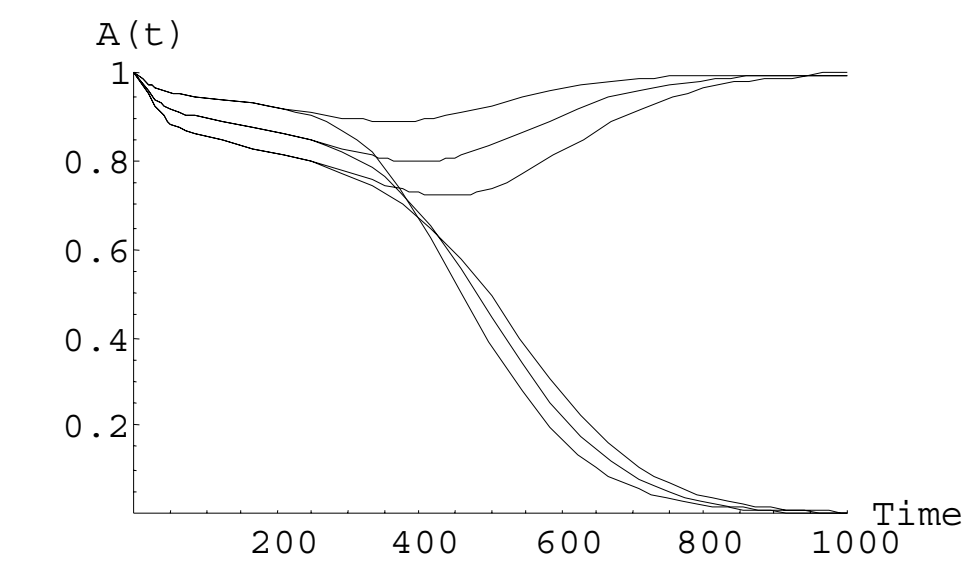

Figure 4.32 Gamma $A_{H}(t)$ with

$$
(k=2, \lambda=2 / 100, a=0.85, r=2, \mu=\{2 / 5,2 / 10,2 / 15\}, b=1.1)
$$

\subsection{Compensation for Computational Limitations}

The limitations on machine arithmetic and number representation impact the numerical results obtained for the quasi-renewal and point availability functions in the case of exponential and gamma operating intervals. A certain amount of error present in the calculations performed and the magnitude of this error prevented the calculation of numerical point availability values for gamma operating intervals with $k \geq 4$. As mentioned earlier, this difficulty is encountered because many of the terms in the point availability functions are lengthy sequences of operations involving $a^{x}$ or $\frac{1}{a^{x}}$ for very large $x$ and $0<a<1$. This is true of the availability function for exponential operating intervals, but is not as severe as the case of the quasi-renewal function and point availability functions for gamma operating intervals. For instance, the downtime based equation for the point availability approximation with gamma operating intervals and gamma repair intervals is 


$$
A^{*}(s)=\frac{1}{S}\left[1-\sum_{n=1}^{c}\left(\left(\frac{\mu+b^{n-1} s}{\mu}\right)^{r}-1\right)\left(\lambda^{k} \mu^{r}\right)^{n} \prod_{i=1}^{n} \frac{1}{\left(\lambda+a^{i-1} s\right)^{k}\left(\mu+b^{i-1} s\right)^{r}}\right] .
$$

Consider just the sixth term that must be inverted

$$
\left(\left(\frac{\mu+b^{6-1} s}{\mu}\right)^{r}-1\right)\left(\lambda^{k} \mu^{r}\right)^{6} \prod_{i=1}^{6} \frac{1}{\left(\lambda+a^{i-1} s\right)^{k}\left(\mu+b^{i-1} s\right)^{r}},
$$

and since the problem occurs for large powers of $a$ consider a much simpler expression and assume $k=4$,

$$
\begin{aligned}
& \prod_{i=1}^{6} \frac{1}{\left(\lambda+a^{i-1} s\right)^{4}}= \\
& \frac{1}{(\lambda+s)^{4}\left(\lambda+a^{1} s\right)^{4}\left(\lambda+a^{2} s\right)^{4}\left(\lambda+a^{3} s\right)^{4}\left(\lambda+a^{4} s\right)^{4}\left(\lambda+a^{5} s\right)^{4}} .
\end{aligned}
$$

Without expanding the denominator it is easy to see that the final term is $a^{60} s^{24}$. Upon inverting the true expression in 4.8 and then summing all $c$ terms, the expression of the availability approximation in the time domain gets rather complex and requires multiplication and division involving hundreds of terms that include $a$ raised to a large power. Therefore, it is essential that the Laplace transforms be inverted symbolically rather than numerically. The numerical inversion of the Laplace transforms adds another source of error that make difficulties in obtaining numerical values for the quasi-renewal and point availability functions even worse. An example of the symbolic expressions in the time domain can be found in Appendix B. Note that the quasi-renewal and point availability expressions are functions of time after inversion. Large order polynomials involving $a$ operate on the time variable hundreds of times in each of the functions considered. In many instances the individual terms are poorly behaved for smaller values of time, but become rather well behaved as time increases. The rounding error involved with each machine calculation propagates to the final product and causes the behavior shown graphically in Figures 4.36, 4.37, and 4.38. 
Consider the case of exponential operating intervals and exponential repair intervals. The truncated downtime-based point availability function is

$$
A^{*}(s)=\frac{1}{s}-\frac{1}{\mu} \sum_{n=1}^{c} b^{n-1}(\mu \lambda)^{n} \prod_{i=1}^{n} \frac{1}{\left(\lambda+a^{i-1} s\right)\left(\mu+b^{i-1} s\right)} .
$$

Therefore, the form of each individual term that makes a contribution to the value of the availability function can be stated

$$
L^{-1}\left\{\frac{1}{\mu} b^{n-1}(\mu \lambda)^{n} \prod_{i=1}^{n} \frac{1}{\left(\lambda+a^{i-1} s\right)\left(\mu+b^{i-1} s\right)}\right\},
$$

for each of the terms indexed by $n$, where $n=\{1,2, \ldots, c\}$. The third term is examined for a specific set of parameters in Figure 4.33.

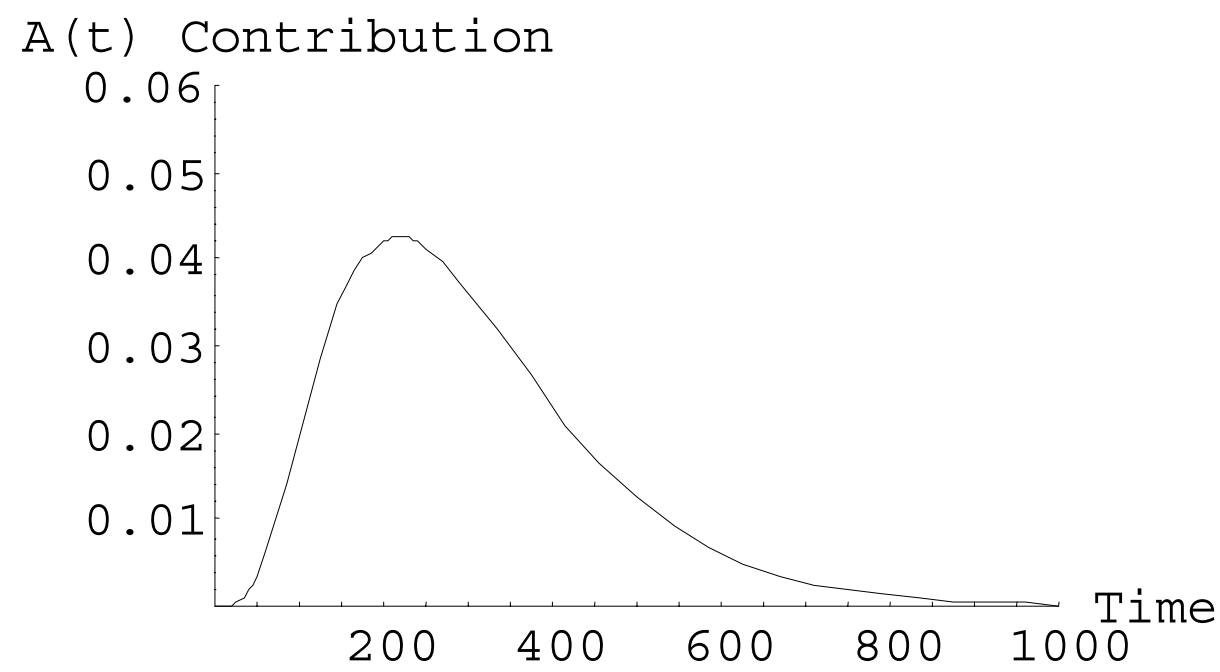

Figure 4.33 Contribution of term 3 to $A_{H}(t)$ with $(\lambda=0.01, a=0.9, \mu=0.1, b=1.2)$

It should be noted that this is actually a negative contribution that decreases the upper bound since it is a term in the downtime-based point availability function. This is well behaved and if the function is observed for small values of time it remains well behaved as shown in Figure 4.34. Note the scale of the availability contribution is for a very small range of values near 0 . 


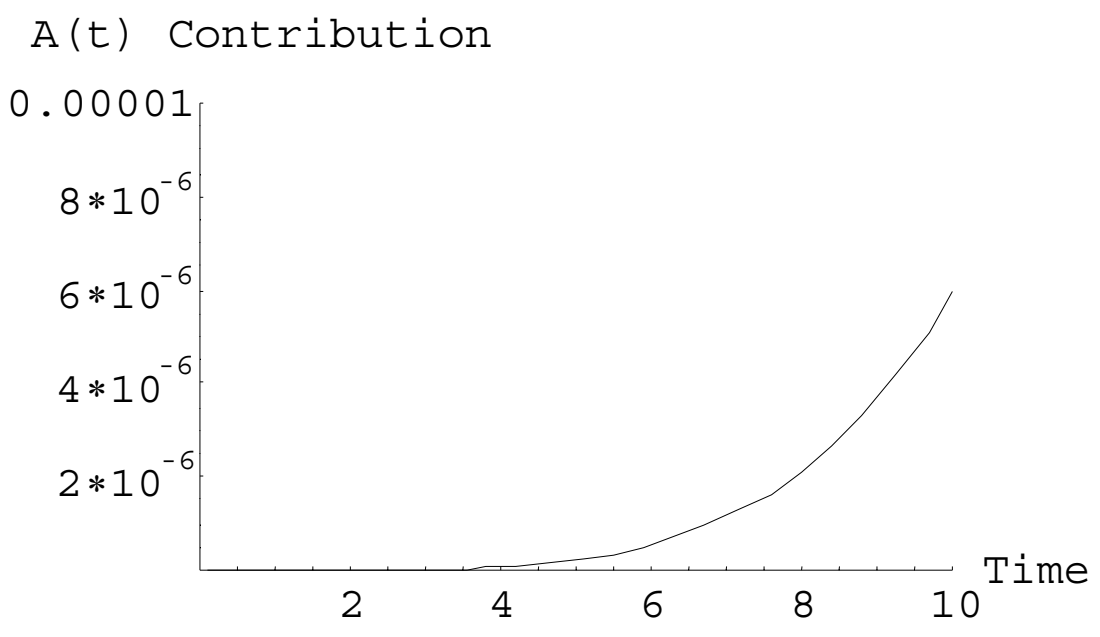

Figure 4.34 Contribution of term 3 to $A_{H}(t)$ with $(\lambda=0.01, a=0.9, \mu=0.1, b=1.2)$

The third term is well behaved since there are relatively few high order polynomials of $a$. Consider the thirteenth term with the same parameter values shown in Figure 4.35.

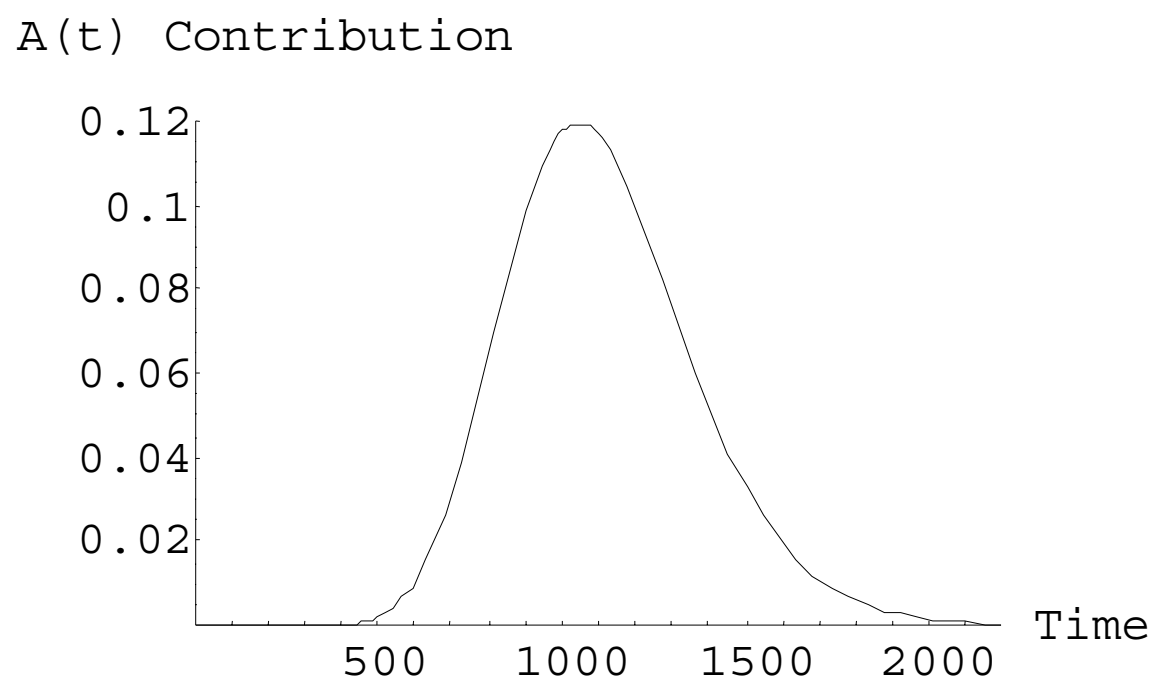

Figure 4.35 Contribution of term 13 to $A_{H}(t)$ with $(\lambda=0.01, a=0.9, \mu=0.1, b=1.2)$

This function appears rather well behaved, but if it is examined for small values of time it is apparent that this is not the case. 


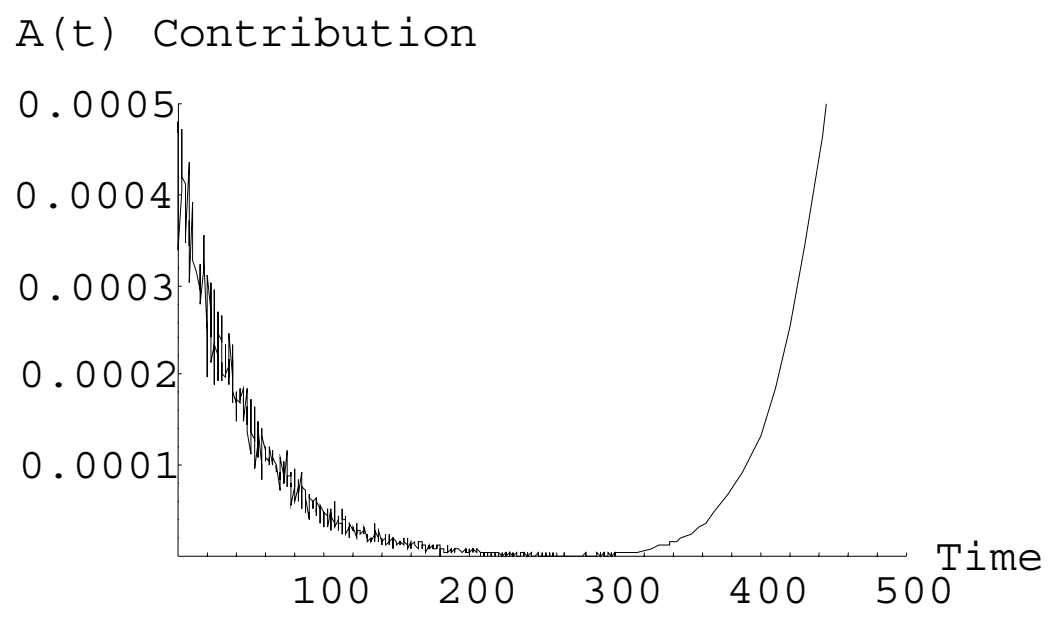

Figure 4.36 Contribution of term 13 to $A_{H}(t)$ with $(\lambda=0.01, a=0.9, \mu=0.1, b=1.2)$

\begin{abstract}
Although this behavior is incorrect and a function of the several factors described above, the absolute impact on the availability function is rather insignificant. At the very worst point this term subtracts 0.0005 from the value of the point availability. Assume that all terms have this degree of error and that they all coincide at the same point in time. The gross impact on the availability of all 13 terms is 0.0065 . However, the behavior shown is not present in the earlier terms as shown above so this is a very conservative worst-case estimate.
\end{abstract}

The behavior described above is more pronounced for the quasi-renewal and point availability functions for gamma operating intervals since the Laplace transform of the gamma distribution is the same as the exponential except it is raised to a power that corresponds to the shape parameter, $k$ (see 3.40 and 3.70). An example of several consecutive contributing terms in the uptime point availability function for gamma operating intervals and exponential repair intervals is shown in Figure 4.37. 


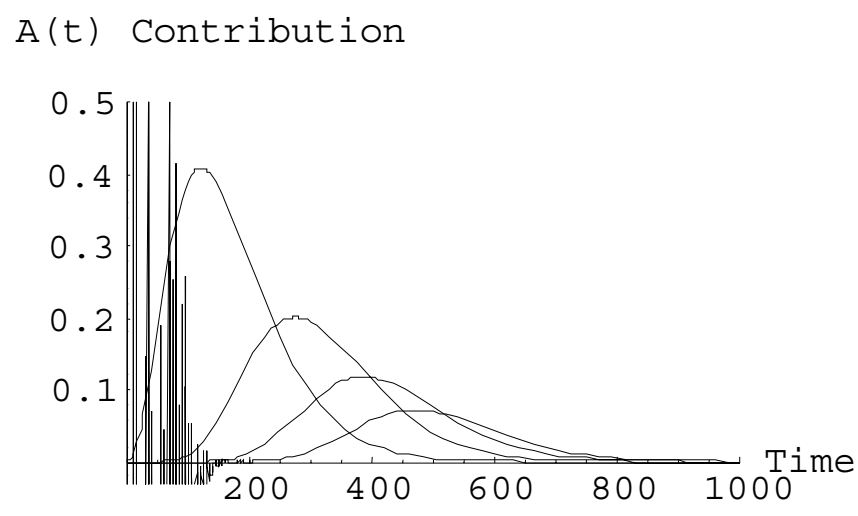

Figure 4.37 Contribution of terms to $A_{H}(t)$ with $(k=2, \lambda=1 / 50, a=0.85, r=1, \mu=0.1, b=1.1)$

Consider the eighth term of the uptime-based point availability function for small values of time in Figure 4.38.

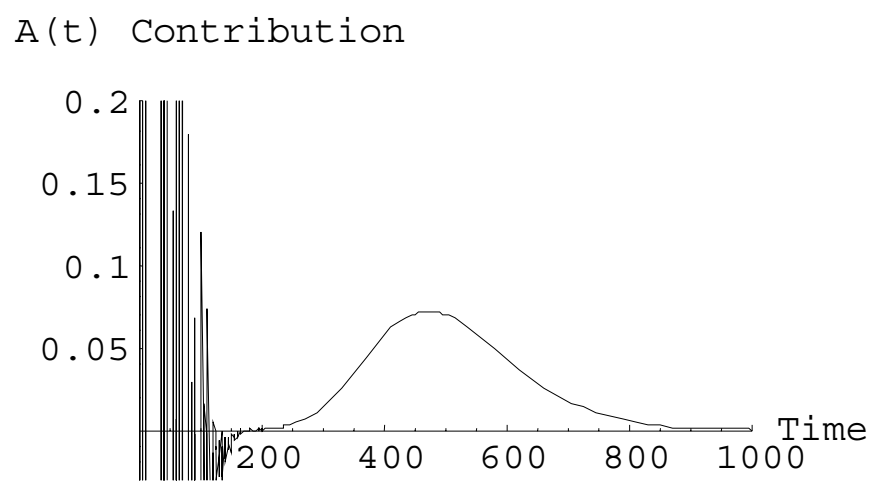

Figure 4.38 Contribution of term 8 to $A_{H}(t)$ with

$$
(k=2, \lambda=1 / 50, a=0.85, r=1, \mu=0.1, b=1.0)
$$

Compared to the error observed in the example with the exponential operating times, the error that is present in calculations for gamma distributed operating intervals is significant. This is especially true if the aggregate impact of the similar behavior for all of the terms used is considered. The maximum precision settings, machine arithmetic settings, and most accurate rounding rules were used to produce the values of the function plotted above. In order to prevent a large amount of accumulated error in the sum that approximates the value of the point availability, the left portion of the function 
is disregarded. In the case above the value of the contribution of the eighth term is considered negligible until it reaches 200 time units. Each term is considered independently and truncated at the appropriate location. This reduces the impact of calculation errors and provides an adequate representation of the availability behavior. Figure 4.39 shows the same terms as in Figure 4.37 with the regions containing large error truncated.

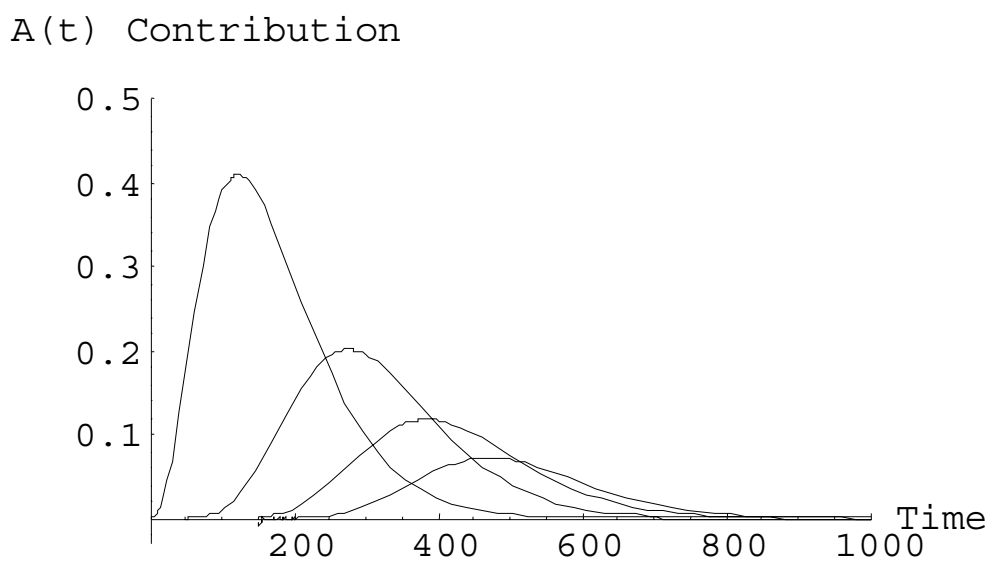

Figure 4.39 Contribution of terms to $A_{H}(t)$ with $(k=2, \lambda=1 / 50, a=0.85, r=1, \mu=0.1, b=1.1)$

The severity of this behavior is influenced by the number of terms evaluated in the infinite sum, the shape parameter of the gamma distribution, the deterioration factor for the operating intervals, and the mean of the first repair interval. As the shape parameter of the gamma distribution increases the error seen above becomes much worse. This behavior also becomes worse as the mean of the first repair interval or the deterioration parameter for the operating intervals becomes too small or too large. The effect for any set of these parameters is cumulative. For instance, if the mean for exponential operating intervals $(k=1)$ is 100 and the mean of the first repair interval is 10 , then the value of the availability function is well behaved at $a=0.9$ for a large number of terms. However, if gamma operating intervals are considered $(k=4)$ with a mean repair for the first operating interval of 10 , then the availability function at $a=0.9$ is impossible to obtain with normal computing resources for more than 4 terms of the infinite sum before the magnitude of the error overwhelms the true value of the function. These consequences are completely 
relative to the number of terms in the infinite sum that are evaluated and the accuracy desired. The error behavior and computing power are the two limiting factors on the depth of analysis possible. It is important to note that the error behavior prevented the calculation of numerical results from terms that were inverted symbolically to the time domain. Therefore, a computer with a larger set of machine values may provide the only solution for obtaining these results. 


\section{Chapter 5 - Conclusions}

This dissertation provides the exact expressions for the point availability and quasirenewal function of a system that deteriorates according to a quasi-renewal process. Deterioration under a quasi-renewal process provides a means to model repair actions that are not quite perfect, but are also not totally ineffective. Therefore, the quasirenewal process is a much more realistic approach in modeling the impact of repair than models based on the minimal repair or perfect repair assumptions. In addition, the behavior of equipment is modeled over time rather than being modeled around limiting behavior. It is interesting to note that the average availability, limiting availability, and limiting average availability are all defined in terms of the point availability. For this reason, it is apparent that the most basic measure of repairable equipment behavior is the point availability and that all other measures are secondary. However, analyses that consider the point availability rather than limiting or average availability are rare. Implied from the statements above, the construction of the point availability is the fundamental first step in truly understanding the availability behavior of any system. It is now possible to examine the time-dependent behavior of a quasi-renewal process and make predictions regarding the performance of a system that deteriorates accordingly. Particularly, it is now possible to define repair and replacement strategies that consider and potentially optimize system point availability over time. This is not possible within the confines of many other non-homogeneous process models.

The development of point availability results for a non-homogeneous process is fairly unique. A detailed framework is provided for expressing and calculating the point availability of a system using the quasi-renewal process to model the deterioration of operating and repair intervals. Specific forms of the point availability and quasi-renewal functions are provided under the assumption of several theoretical distributions on the operating and repair intervals. The quasi-renewal function is used to determine the expected number of system restarts at any point in time under the assumption that repair times are negligible. If repair times are significant, which is often the more realistic 
assumption, then the point availability expressions describe the probability that the system is operational at any point in time.

Two equivalent expressions for the point availability are constructed. These are used to develop bounds on the true value of the point availability when further accurate results are difficult to obtain. The upper bound is useful in another way. It is possible to produce a point availability function with an arbitrary degree of accuracy by causing the value of the upper bound to fall below the desired maximum error. This determines a stopping criterion for the number of terms that must be evaluated in the infinite sum expressions for the point availability. However, computational resources must be sufficient to evaluate enough terms to drive the upper bound on the point availability near 0 to exploit this property.

Numerical results under the assumption of repair and operating intervals that deteriorate according to a quasi-renewal process provide insight into the behavior of the point availability and renewal-type functions for a non-homogeneous process. As mentioned earlier, modeling efforts that do not rely on limiting behavior or that assume minimal repair or renewal is unfortunately uncommon. This effort provides analytical results in the very interesting and realistic class of problems that do not make any of these assumptions. Future research that considers more flexible models for non-homogeneous processes is necessary.

Both computational power and the precision of machine arithmetic are issues in obtaining numerical results for the quasi-renewal and point availability functions. In order to obtain useful results, the Laplace transforms must be inverted symbolically which is computationally demanding. Following symbolic inversion, the resulting expression in the time domain must be solved for particular parameter values. The nature of the expressions in the time domain are such that the rounding error resulting from machine arithmetic involving very large and very small numbers induces a certain amount of error in the numerical results. This becomes more or less pronounced depending on the combination of parameter values that are used. Typically, as the 
deterioration factor of the operating or repair intervals increases, corresponding to slowly deteriorating operating intervals or quickly deteriorating repair intervals, the machine error becomes worse. In addition, the use of theoretical distributions with an exponential form, such as the gamma distribution, amplifies this behavior. In some cases the machine error masks the true behavior of the function and prevents the calculation of numerical results for symbolic expressions obtained.

Some general statements can be made about the sensitivity of the point availability functions to certain parameters for gamma and exponentially distributed operating intervals. When a repair parameter is altered it causes a distinct and predictable shift in the upper bound, but the behavior of the lower bound is altered in a more subtle fashion. This is because the upper bound is calculated using the downtime-based point availability approximation, which is more dependent upon the repair distribution than the distribution of the operating intervals. Similarly, changes in an operation interval parameter causes a distinct and predictable shift in the lower bound since the lower bound is calculated using the downtime-based point availability function, which is more dependent upon the distribution on the operating intervals. 


\section{Chapter 6 - Future Research}

The construction of point availability and quasi-renewal functions for specific theoretical distributions on operation and repair intervals demonstrates the potential for the practical application of the quasi-renewal process in the modeling and analysis of equipment behavior. Further exploration of the time-dependent behavior of nonhomogeneous models is necessary to correctly model the majority of situations encountered in practice. This analysis of the quasi-renewal model is a step in this direction. However, a similar study of other non-homogeneous models, such as Kijima's [1988] virtual age model should be undertaken.

In addition to the continued study of the time-dependent availability behavior of non-homogeneous processes in general, there are several opportunities for direct extension of the work that is contained in this dissertation. One very important consideration is the development of preventive maintenance strategies to increase the availability performance of a system under the assumption of quasi-renewal operating intervals and repair intervals. The point availability and quasi-renewal functions described have value in making predictions regarding the behavior of a system. However, the construction of an optimal preventive maintenance strategy makes use of the predictive power that is now possible to modify system performance in a positive direction. For example, the point at which preventive maintenance is warranted may be defined in terms of a threshold value for the point availability. In addition, the time at which replacement is more desirable than preventive maintenance should be addressed.

If this model is to be used in practice, it is necessary to develop a method for estimating the deterioration parameters and distribution parameters for the operation and repair intervals from existing process data. A method for parameter estimation indicates the data collection efforts that are necessary and facilitates implementation of the quasirenewal model. 
The normal, gamma, and exponential distributions are examined as candidate distributions for the intervals of operation. However, the Weibull distribution is the most widely used distribution in reliability and availability analyses. For this reason the point availability and quasi-renewal functions should be constructed under the assumption of Weibull distributed operating intervals. Lomnicki's [1966] method for approximating the Weibull distribution may be the most promising avenue of exploration to this end.

In addition to incorporating the Weibull distribution, an effort should be made to determine the conditions under which the normal distribution can serve as an adequate approximation for other theoretical distributions on the length of operating intervals. The tractability and ease of numerical computation in the case of normal operating intervals should be fully exploited.

Finally, computing power and machine rounding error are the two most significant limitations on the ability to provide more extensive numerical calculations. Mainframe computing resources may provide the capability to symbolically invert the infinite expressions for the point availability and quasi-renewal functions to a greater degree. If this capability is coupled with a method to reduce machine rounding error in the numerical calculations of the inverted expressions it may be possible to compute near exact expressions for the point availability function in the cases of the exponential and gamma distributed operating intervals. 


\section{References}

1. Ascher, H. (1968) "Evaluation of Repairable System Reliability Using the 'Bad-asOld' Concept," IEEE Transactions on Reliability, R-17, 103-110.

2. Ascher, H. and Feingold, H. (1984) Repairable Systems Reliability, Marcel Dekker, Inc., New York.

3. Abdel-Hameed, M. (1995) "Inspection, Maintenance and Replacement Models," Computers and Operations Research, Vol. 22, No. 4, pp. 435-441.

4. Abramowitz, M. and Stegun, I. A. (1972) "Handbook of Mathematical Functions," Dover Publications, Inc., New York.

5. Balaban, H. S. (1978) "A Stochastic Characterization of Failure Processes under Minimal Repair," Unpublished Ph. D. Dissertation, George Washington University.

6. Barlow, R. and Hunter, L. (1960) "Optimum Preventive Maintenance Policies," Operations Research, Vol. 8, pp. 90-100.

7. Barlow, R. and Proschan, F. (1967) Mathematical Theory of Reliability, John Wiley \& Sons, Inc., New York.

8. Barlow, R. and Proschan, F. (1975) Statistical Theory of Reliability and Life Testing, Holt, Rinehart, and Winston, Inc., New York

9. Barlow, R. and Proschan, F. (1981) Statistical Theory of Reliability and Life Testing, To Begin With, Silver Spring, Maryland.

10. Bassin, W. M. (1969) "Increasing Hazard Functions and Overhaul Policy," Proceedings of the Annual Reliability and Maintainability Symposium, pp. 173-180.

11. Baxter, L. A., Kijima, M., and Tortorella, M. (1996) "A Point Process Model for the Reliability of a Maintained System Subject to General Repair," Communications in Statistics, Vol. 12, No. 1, pp. 37-65.

12. Berg, M. and Epstein, B. (1976) "A Modified Block Replacement Policy," Naval Research Logistics Quarterly, Vol. 23, pp. 15-24.

13. Block, H. W., Borges, W. S., Savits, T. H. (1985) "Age-Dependent Minimal Repair," Journal of Applied Probability, Vol. 22, pp. 370-385.

14. Brown, M. and Proschan, F. (1983) "Imperfect Repair," Journal of Applied Probability, Vol. 20, pp. 851-859. 
15. Cassady, C. R., Maillart, L. M., Bowden, R. O., and Smith B. K. (1998) "Characterization of Optimal Age-Replacement Policies," Proceedings of the Annual Reliability and Maintainability Symposium, pp. 170-175.

16. Chan, J. K. and Shaw, L. (1993) "Modeling Repairable Systems with Failure Rates that Depend on Age and Maintenance," IEEE Transactions on Reliability, Vol. 42, No. 4, pp. 566-571.

17. Clarke, A. B. and Disney, R. L. (1985) Probability and Random Processes: A First Course with Applications, John Wiley and Sons, Inc.

18. Cox, D. (1962) Renewal Theory, Methuen and Co., Ltd., London.

19. Dagpunar, J. S. (1997) "Renewal-Type Equations for a General Repair Process," Quality and Reliability Engineering International, Vol. 13, pp. 235-245.

20. Dagpunar, J. S. (1998) "Some Properties and Computational Results for a General Repair Process," Naval Research Logistics, Vol. 45, pp. 391-405.

21. Daigle, J. N. (1992) Queueing Theory for Telecommunications, Addison-Wesley Publishing Co., Inc.

22. Engelhardt, M. and Bain, L. J. (1986) "On the Mean Time Between Failures For Repairable Systems," IEEE Transactions on Reliability, Vol. R-35, No. 4, pp. 419422.

23. Esary, J. D., Marshall, A. W., and Proschan, F. (1973) "Shock Models and Wear Processes," Annals of Probability, Vol. 1, pp. 627-650.

24. Finkelstein, M. S. (1993a) "A Scale Model of General Repair," Microelectronics Reliability, Vol. 33, No. 1, pp. 41-44.

25. Finkelstein, M. S. (1993b) "On Some Models of General Repair," Microelectronics Reliability, Vol. 33, No. 5, pp. 663-666.

26. Flehinger, B. (1962) "A General Model for the Reliability of Systems under Various Preventive Maintenance Policies," The Annals of Mathematical Statistics, Vol. 33, No. 1, pp. 137-146.

27. Hoel, P. G., Port, S. C., and Stone, C. J. (1971) "Introduction to Probability Theory," Houghton Mifflin Company, Boston.

28. Iyer, S. (1992) "Availability Results for Imperfect Repair," Sankhya: The Indian Journal of Statistics, Vol. 54, Series B, Pt. 2, pp. 249-256. 
29. Kijima, M., Morimura, H., and Suzuki, Y. (1988) "Periodical Replacement Problem without Assuming Minimal Repair," European Journal of Operational Research, Vol. 37, pp. 194-203.

30. Kijima, M. (1989) "Some Results for Repairable Systems with General Repair," Journal of Applied Probability, Vol. 26, pp. 89-102.

31. Kijima, M. and Nakagawa, T. (1992) "Replacement Policies of a Shock Model with Imperfect Preventive Maintenance," European Journal of Operational Research, Vol. 57, pp. 100-110.

32. Lam Yeh (1988) "A Note on the Optimal Replacement Problem," Advances in Applied Probability, Vol. 20, pp. 479-482.

33. Lam Yeh (1990) "A Repair Replacement Model," Advances in Applied Probability, Vol. 22, pp. 494-497.

34. Lemoine, A. J. and Wenocur, M. L. (1985) "On Failure Modeling," Naval Research Logistics Quarterly, Vol. 32, pp. 497-508.

35. Lemoine, A. J. and Wenocur, M. L. (1986) "A Note On Shot-Noise and Reliability Modeling," Operations Research, Vol. 34, No. 2, pp. 320-323.

36. Lie, C. H. and Chun, Y. H. (1986) "An Algorithm for Preventive Maintenance Policy," IEEE Transactions on Reliability, Vol. R-35, No. 1, pp. 71-75.

37. Lomnicki, Z. A. (1966) "A Note on the Weibull Renewal Process," Biometrika, Vol. 53, pp. 375-381.

38. Luss, H. (1976) "Maintenance Policies when Deterioration can be Observed by Inspection," Operations Research, Vol. 24, pp. 359-366.

39. Makis, V. and Jardine, K. S. (1993) "A Note on Optimal Replacement Policy under General Repair," European Journal of Operational Research, Vol. 69, pp. 75-82.

40. Malik, M. A. K. (1979) "Reliable Preventive Maintenance Scheduling," AIIE Transactions, Vol. 11, pp. 221-228.

41. McCall, J. (1965) "Maintenance Policies for Stochastically Failing Equipment: A Survey," Management Science, Vol. 11, No. 5, pp. 493-524.

42. Murdock, W. (1995) "Component Availability for an Age Replacement Preventive Maintenance Policy," Unpublished Ph.D. Dissertation, Virginia Polytechnic Institute and State University, Department of Industrial and Systems Engineering. 
43. Murthy, D. N. P., and Nguyen, D. G. (1982) "A Note on Extended Block Replacement Policy with Used Items," Journal of Applied Probability, Vol. 19, pp. 885-889.

44. Murthy, D. N. P. (1991) "A Note on Minimal Repair," IEEE Transactions on Reliability, Vol. 40, No. 2, pp. 245-246.

45. Nachlas, J. A. (1989) "Availability Distribution Based Preventive-Maintenance Strategies," International Journal of Modeling and Simulation, Vol. 9, pp. 49-52.

46. Nachlas, J. A. and Cassady, C. R. (1999) "Preventive Maintenance Study: A Key Component in Engineering Education to Enhance Industrial Productivity and Competitiveness," European Journal of Engineering Education, Vol. 24, No. 3, pp. 299-309.

47. Osaki, S. and Nakagawa, T. (1976) "Bibliography for Reliability and Availability of Stochastic Systems," IEEE Transactions on Reliability, Vol. R-25, pp. 284-287.

48. Pham, H. and Wang, H. (1996) "Imperfect Maintenance," European Journal of Operational Research, Vol. 94, pp. 425-438.

49. Pierskalla, W. and Voelker, J. (1976) "A Survey of Maintenance Models: The Control and Surveillance of Deteriorating Systems," Naval Research Logistics Quarterly, Vol. 23, pp. 353-388.

50. Rangan, A. and Sarada, G. (1992) "Some Results on the Life Distribution Properties of Systems Subject to Shocks and General Repair," Microelectronics Reliability, Vol. 33, No. 1, pp. 1-6.

51. Reineke, D., Murdock, W., Pohl, E., and Rehmert, I. (1999) "Improving Availability and Cost Performance for Complex Systems with Preventive Maintenance," Proceedings of the Annual Reliability and Maintainability Symposium, pp. 383-388.

52. Ross, S. M. (1981) "Generalized Poisson Shock Models," Annals of Probability, Vol. 9, pp. 896-898.

53. Sandve, K. and Aven, T. (1999) "Cost Optimal Replacement of Monotone, Repairable Systems," European Journal of Operational Research, Vol. 116, pp. 235248.

54. Shaked, M. and Shanthikumar, J. G. (1986) "Multivariate Imperfect Repair," Operations Research, Vol. 34, No. 3, pp. 437-448. 
55. Shaked, M. and Zhu, H. (1992) "Some Results on Block Replacement Policies and Renewal Theory," Journal of Applied Probability, Vol. 29, pp. 932-946.

56. Shanthikumar, J. G. and Sumita, U. (1983) "General Shock Models Associated with Correlated Renewal Sequences," Journal of Applied Probability, Vol. 20, pp. 600614.

57. Sherif, Y. and Smith, M. (1981) "Optimal Maintenance Models for Systems Subject to Failure - A Review," Naval Research Logistics Quarterly, Vol. 28, pp. 47-74.

58. Shue, S. H. and Griffith, W. S. (1992) "Multivariate Imperfect Repair," Journal of Applied Probability, Vol. 29, pp. 947-956.

59. Shue, S. H. (1993) "A Generalized Model for Determining Optimal Number of Minimal Repairs before Replacement," European Journal of Operational Research, Vol. 69, pp. 38-49.

60. Shue, S. H. (1994) "Extended Block Replacement Policy with Used Item and General Random Repair Cost," European Journal of Operational Research, Vol. 79, pp. 405416.

61. Shue, S. H. (1996) "A Modified Block Replacement Policy with Two Variables and General Random Minimal Repair Cost," Journal of Applied Probability, Vol. 33, pp. 557-572.

62. Shue, S. H. (1998) "A Generalized Age and Block Replacement of a System Subject to Shocks," European Journal of Operational Research, Vol. 108, pp. 345-362.

63. Spiegel, M. R. (1965) "Schaum's Outline of Theory and Problems of Laplace Transforms," McGraw-Hill, New York.

64. Stadje, W. and Zuckerman, D. (1991) "Optimal Maintenance Strategies for Repairable Systems with General Degree of Repair," Journal of Applied Probability, Vol. 28, pp. 384-396.

65. Tango, T. (1978) "Extended Block Replacement Policy with Used Item," Journal of Applied Probability, Vol. 15, pp. 560-572.

66. Valdez-Flores, C. and Feldman, R. (1989) "A Survey of Preventive Maintenance Models for Stochastically Deteriorating Single-Unit Systems," Naval Research Logistics, Vol. 36, pp. 419-446. 
67. Vaurio, J. K. (1999) "Availability and Cost Functions for Periodically Inspected Preventively Maintained Units," Reliability Engineering and Systems Safety, Vol. 63, pp. 133-140.

68. Wang, H. and Pham, H. (1996a) "Optimal Maintenance Policies for Several Imperfect Repair Models," International Journal of Systems Science, Vol. 27, No. 6, pp. 543549.

69. Wang, H. and Pham, H. (1996b) "A Quasi-renewal Process and its Applications in Imperfect Maintenance," International Journal of Systems Science, Vol. 27, No. 10, pp. 1055-1062.

70. Wang, H. and Pham, H. (1997) "Changes to: 'A Quasi-renewal Process and its Applications in Imperfect Maintenance'," International Journal of Systems Science, Vol. 28, No. 12, pp. 1329.

71. Wortman, M. A., Klutke, G., and Ayhan, H. (1994) "A Maintenance Strategy for Systems Subjected to Deterioration Governed by Random Shocks," IEEE Transactions on Reliability, Vol. 43, No. 3, pp. 439-445.

72. Zhang, F. and Jardine, A. K. S. (1998) "Optimal Maintenance Models with Minimal Repair, Periodic Overhaul, and Complete Renewal," IIE Transactions, Vol. 30, pp. 1109-1119.

73. Zuckerman, D. (1980) "Inspection and Replacement Policies," Journal of Applied Probability, Vol. 17, pp. 168-177. 


\section{Appendix A - Sample Mathematica Input}

\section{A.1 Exponential $(\lambda)$ Operating \& Exponential $(\mu)$ Repair}

(* this section computes each term neoessary to cormoute the UPTIM-

BASED POINI AVATIABIIITY of a system with quasi-

renewal operating times distributed with rate $\lambda$ and deterioration factor $a$,

and QUASI-RENEWAL REPAIR TIMES distributed with rate $\mu$ and deterioration factor b. Each

term can be added to precoding availability to generate a more accurate availability expression *)

InverselaplaceTransform $\left[\frac{1}{\lambda+s}, s, t\right]$

$\mathrm{Do}\left[\right.$ Print [InverselaplacoIransform $\left.\left.\left[\frac{(a \mu \lambda)^{n}}{\lambda+a^{n} s} \prod_{1=1}^{n} \frac{1}{\left(\lambda+a^{1-1} s\right)\left(\mu+b^{1-1} s\right)}, s, t\right]\right],\{n, 20\}\right]$

(* this section computes each term neoessary to compute the DowNIIME-

BASED POINI AVATIABIITIY of a system with quasi-

renewal operating times distributed with rate $\lambda$ and deterioration factor $a$,

and QUASI-RENEWAL REPAIR TMES distributed with rate $\mu$ and deterioration factor $b$. Each

term can be added to precoding availability to generate a more accurate availability expression *)

DoI

InverselaplaceTransform $\left[(b \mu)^{n-1} \lambda^{n} \prod_{1=1}^{n} \frac{1}{\left(\lambda+a^{i-1} s\right)\left(\mu+b^{i-1} s\right)}, s, t\right]$;

Print $[\mathrm{n}]$;

$,\{n, 30\}]$ 


\section{A.2 Normal $(\mu, \sigma)$ Operating \& $\operatorname{Normal}(\lambda, \rho)$ Repair}

(* THIS SECTION COMPUTES THE UPTIME-BASED AVAIIABHITTY FOR NORMAL QUASI-

RENEWAL OPERATING INIERVAIS AND NOFMAL QUASI-RENEWAL REPAIR INIERVAIS *)

$\ll$ Statistics 'ContinuousDistributions`

quasinomalrep $\left[\mu, \sigma_{-}, a_{-}, \lambda_{-}, \rho_{-}, b_{-}, t_{-}, l_{1}\right]:=$

$1-\operatorname{CDF}[$ NormalDistribution $[\mu, \sigma], t]+$

$$
\begin{aligned}
& \sum_{n=1}^{\lim }\left(\operatorname{CDF}\left[\text { NormalDistribution }\left[\frac{\mu\left(1-a^{n}\right)}{1-a}+\lambda \sum_{i=1}^{n} b^{i-1}, \sqrt{\frac{\sigma^{2}\left(1-a^{2 n}\right)}{\left(1-a^{2}\right)}+\rho^{2} \sum_{i=1}^{n} b^{2}(i-1)}\right], t\right]-\right. \\
& \left.\quad \operatorname{CDF}\left[\text { NormalDistribution }\left[\mu a^{n}+\frac{\mu\left(1-a^{n}\right)}{1-a}+\lambda \sum_{i=1}^{n} b^{i-1}, \sqrt{\sigma^{2} a^{2 n}+\frac{\sigma^{2}\left(1-a^{2}\right)}{\left(1-a^{2}\right)}+\rho^{2} \sum_{i=1}^{n} b^{2(i-1)}}\right], t\right]\right)
\end{aligned}
$$

(* THIS SECIION COMPUTES THE DONNTTME-BASED AVATIABIIITY FOR NORMAL QUASIRENEENAL OPERATTING TNIEKRVAIS AND NORMAL QUASI-

RENEWAL REPATR INIERVAIS -- SINCE THIS IS THE UPEER BOUND ON THE AVATIABIITIY, WHEN THIS GOES TO ZWBRO WE KNOW WE HAVE A VERY ACCURATE AVAIIABIIITTY EUNCIION *)

quasinormalrepdowntime $\left[\mu_{-}, \sigma_{-}, a_{-}, \lambda_{-}, \rho_{-}, b_{-}, t_{-}, l_{1 i m}\right]:=$

1 -

$$
\begin{aligned}
& \sum_{n=1}^{\lim }\left(\operatorname{CDF}\left[\text { NomalDistribution }\left[\frac{\mu\left(1-a^{n}\right)}{1-a}+\lambda \sum_{i=1}^{n-1} b^{i-1}, \sqrt{\frac{\sigma^{2}\left(1-a^{2 n}\right)}{\left(1-a^{2}\right)}+\rho^{2} \sum_{i=1}^{n-1} b^{2}(i-1)}\right], t\right]\right. \\
& \quad \operatorname{CDE}\left[\text { NormalDistribution } \left[\frac{\mu\left(1-a^{n}\right)}{1-a}+\lambda \sum_{i=1}^{n} b^{i-1}, \sqrt{\left.\left.\left.\frac{\sigma^{2}\left(1-a^{2 n}\right)}{\left(1-a^{2}\right)}+\rho^{2} \sum_{i=1}^{n} b^{2(i-1)}\right], t\right]\right)}\right.\right.
\end{aligned}
$$




\title{
A.3 Gamma $(2, \lambda)$ Operating \& Exponential $(\mu)$ Repair
}

\author{
< NumericalMath 'CamputerArithmetic' \\ SetArithmetic $[10,10$, RoundingRule $\rightarrow$ RoundroInfinity, ExponentRange $\rightarrow\{-1000,1000\}$, \\ Mixed Moda $\rightarrow$ False, IchalDivide $\rightarrow$ False] \\ $\{10,10$, RoundingRule $\rightarrow$ RoundToInfinity, \\ ExponentRange $\rightarrow\{-1000,1000\}$, MixedMode $\rightarrow$ False, IdealDivide $\rightarrow$ False $\}$
}

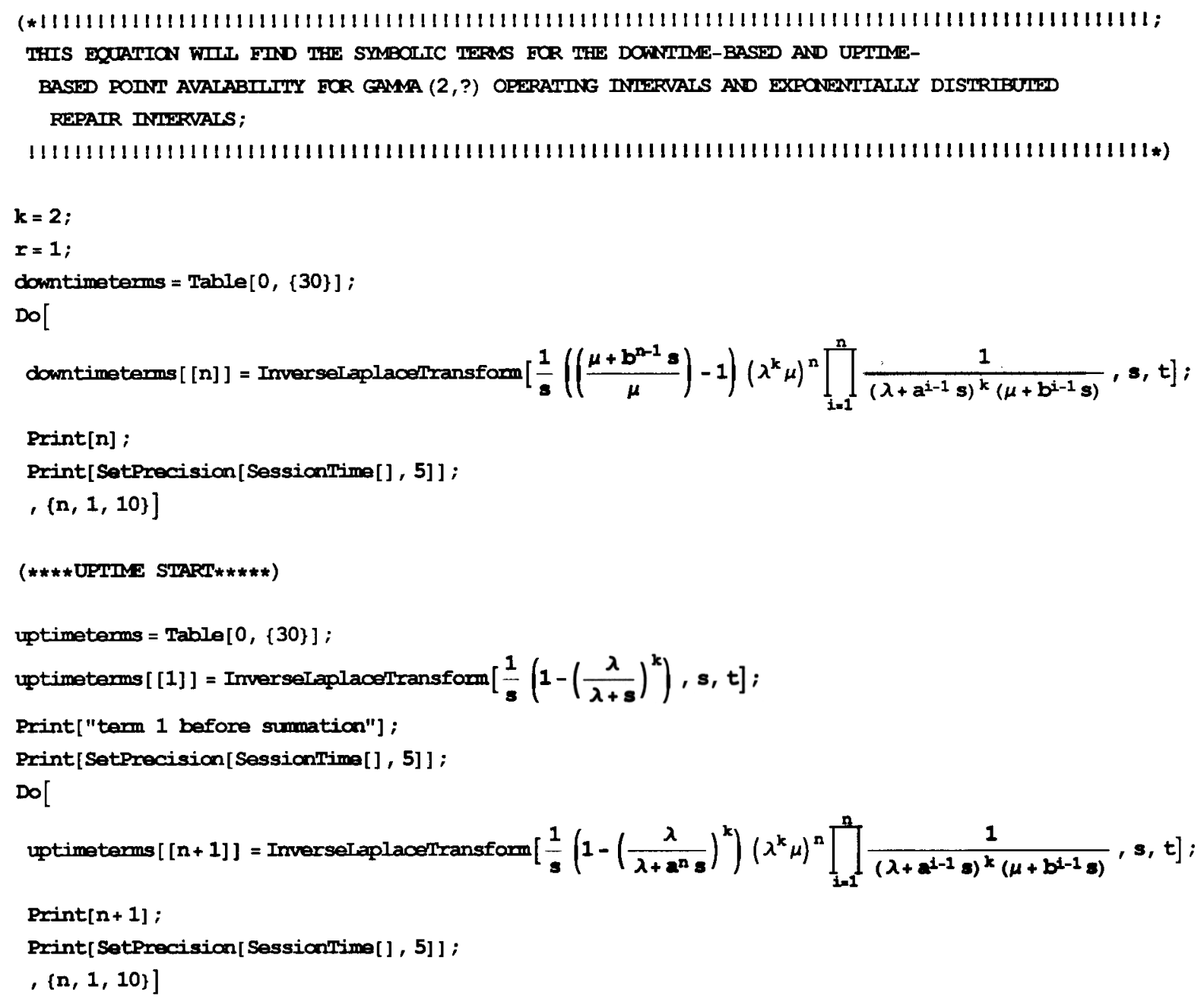




\section{A.4 Gamma $(2, \lambda)$ Operating \& Gamma $(2, \mu)$ Repair}

$\ll$ NumaricalMath 'ComputerArithmetic'

SetArithmetic $[10,10$, RoundingRule $\rightarrow$ RoundroInfinity, EqponentRange $\rightarrow\{-1000,1000\}$, Mixediode $\rightarrow$ False,

Idabivivida $\rightarrow$ False]

$\{10,10$, RoundingRule $\rightarrow$ RoundToInfinity, ExponentRange $\rightarrow\{-1000,1000\}$, MixedMode $\rightarrow$ False, IdealDivide $\rightarrow$ False $\}$

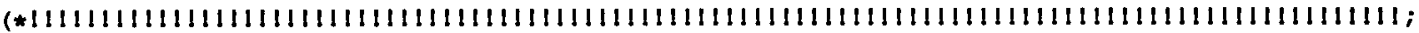
THIS EQTATION WILL FIND THE SMMOTIC TEFPS FOR TFE DOTNIME-BASED AND UPTIME-

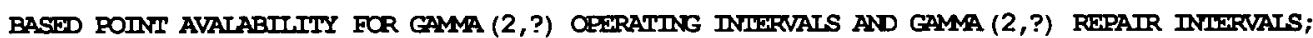

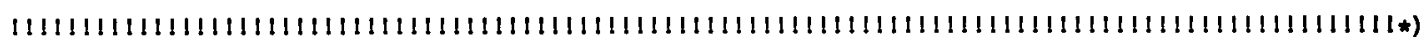

$\mathbf{k}=2$;

$r=2$;

downtimeterns $=$ Table $[0,\{30\}]$;

Do[

downtimeterms $[n]]=$ Inverselaplacolransform $\left[\frac{1}{s}\left(\left(\frac{\mu+b^{n-1} s}{\mu}\right)^{x}-1\right)\left(\lambda^{k} \mu^{x}\right)^{n} \prod_{i=1}^{n} \frac{1}{\left(\lambda+a^{i-1} s\right)^{k}\left(\mu+b^{d-1} s\right)^{x}}, s, t\right] ;$ Print $[n]$;

Print [SetPrecision[SessionTima [], 5] ] ;

$,\{n, 1,7\}]$

$(* * * *$ UPIIME START $* * * * *)$

Lptimatanns $=$ Table $[0,\{30\}]$;

uptimeterns $[[1]]=$ IrvarselaplaceTransform $\left[\frac{1}{s}\left(1-\left(\frac{\lambda}{\lambda+s}\right)^{k}\right), s, t\right]$;

Print["tarm 1 before sumation"] ;

Print [SetPrecision [ SessianTime[], 5] ] ;

Do[

uptimeterms $[n+1]]=$ InverseLaplacetransform $\left[\frac{1}{s}\left(1-\left(\frac{\lambda}{\lambda+a^{n} s}\right)^{k}\right)\left(\lambda^{k} \mu^{r}\right)^{n} \prod_{1=1}^{n} \frac{1}{\left(\lambda+a^{d-1} s\right)^{k}\left(\mu+b^{d-1} s\right)^{x}}, s, t\right]$;

Print $[n+1]$;

Print [SetPrecision [SessionTime [] , 5] ] ;

$,(n, 1,7)]$ 


\section{Appendix B - Sample Inverted Laplace Transforms}

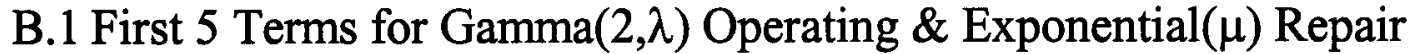

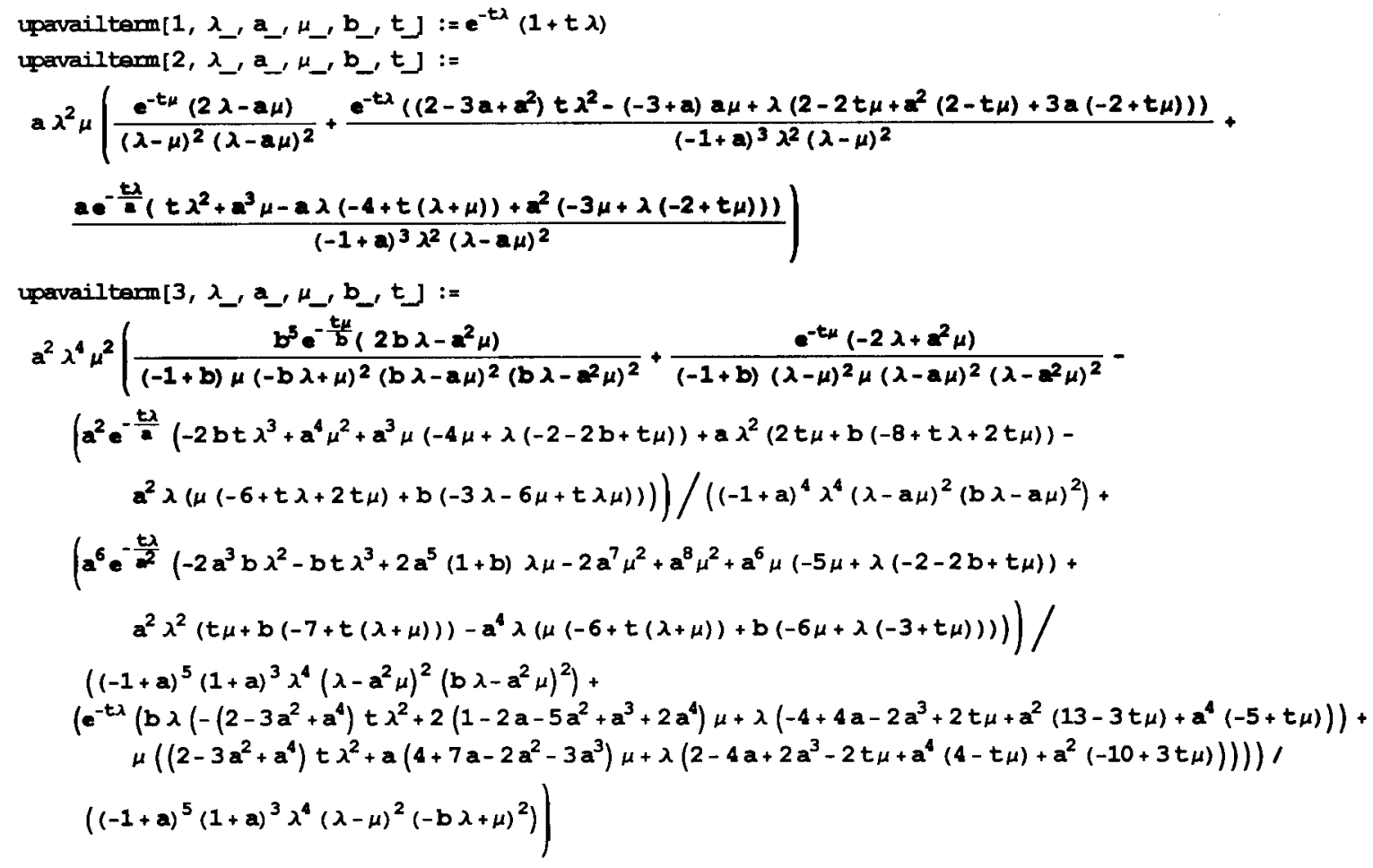


upavailterm $\left[4, \lambda_{-}, a_{-}, \mu_{-}, b_{-}, t_{-}\right]:=$

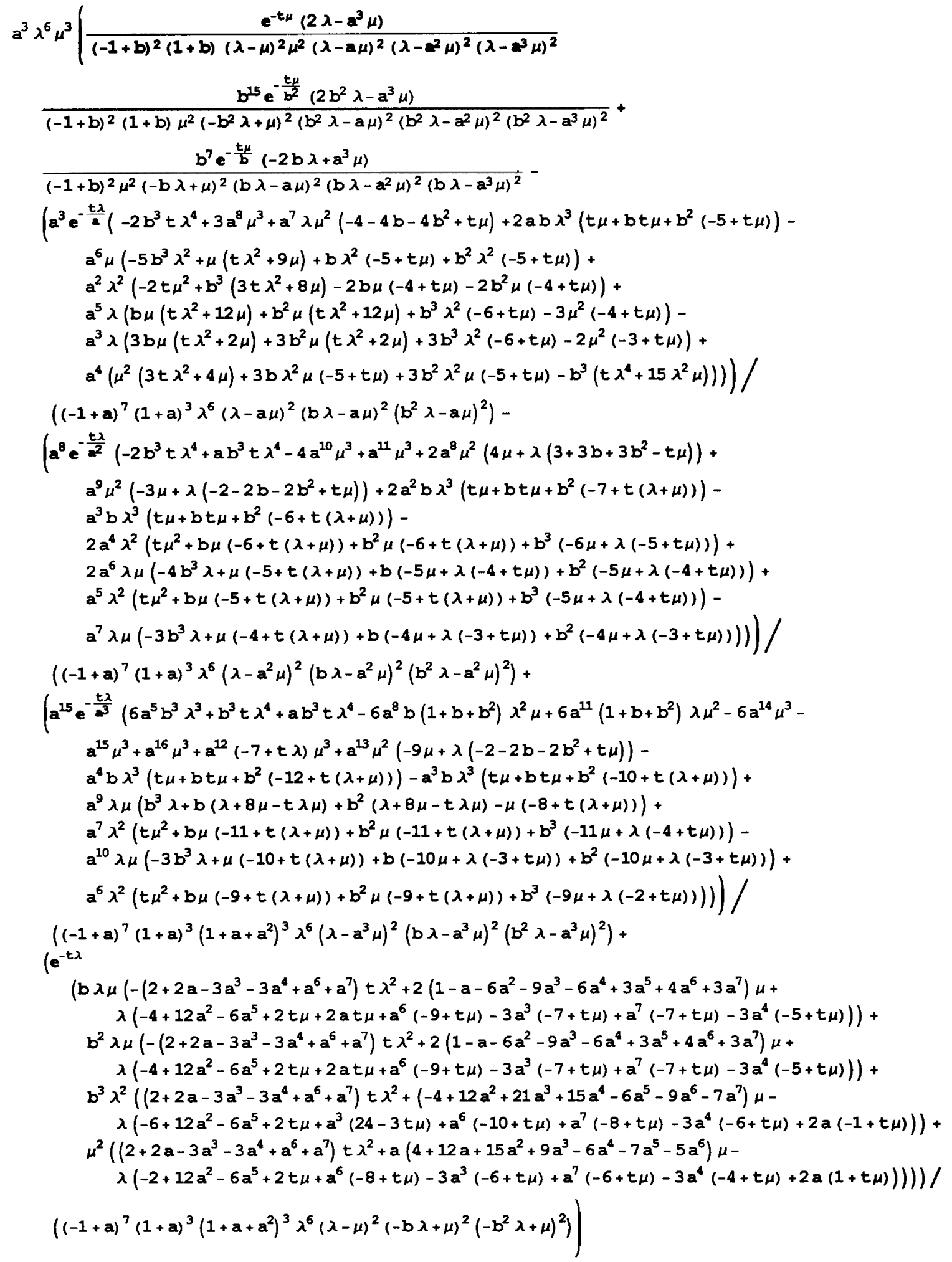




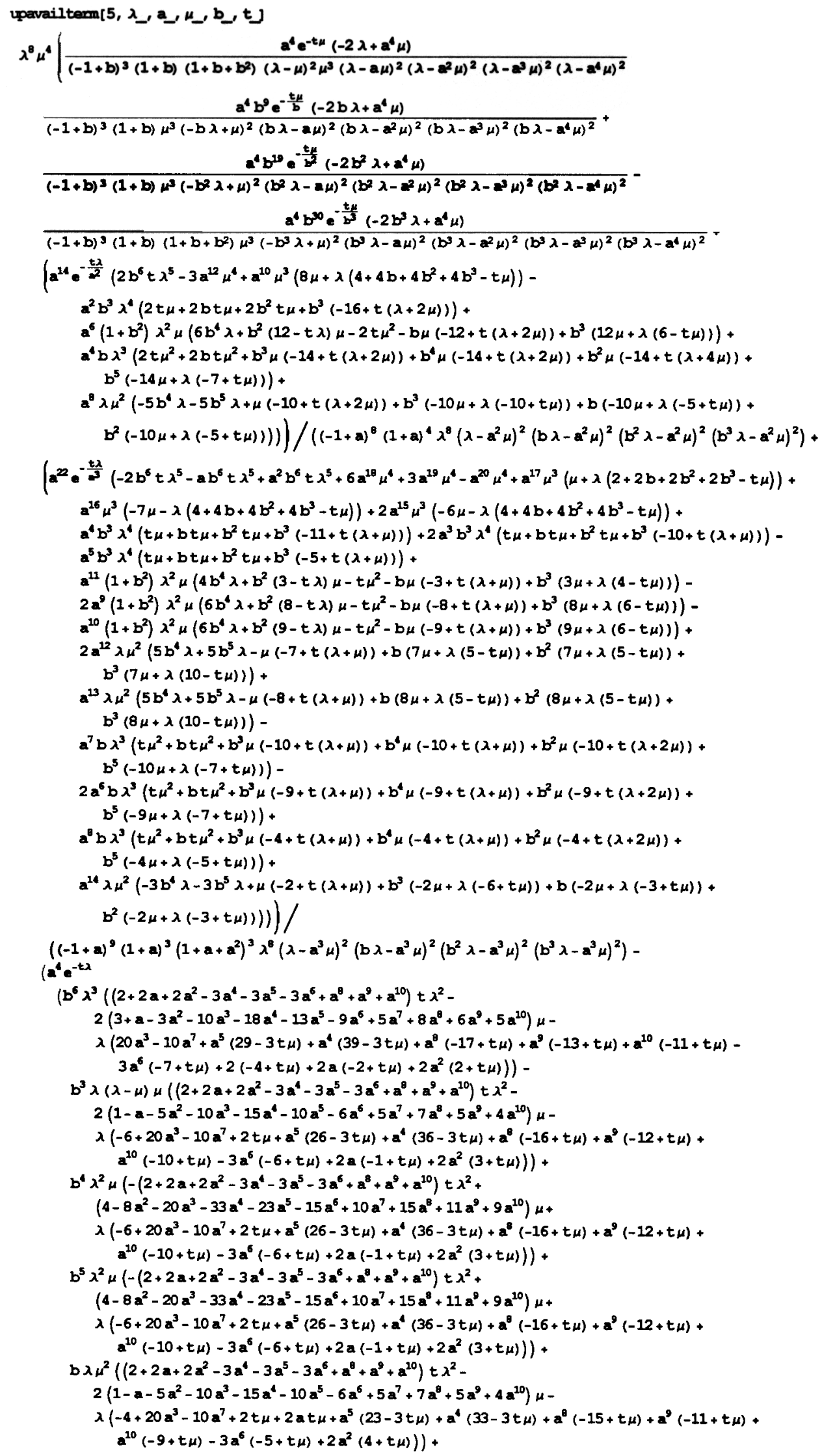




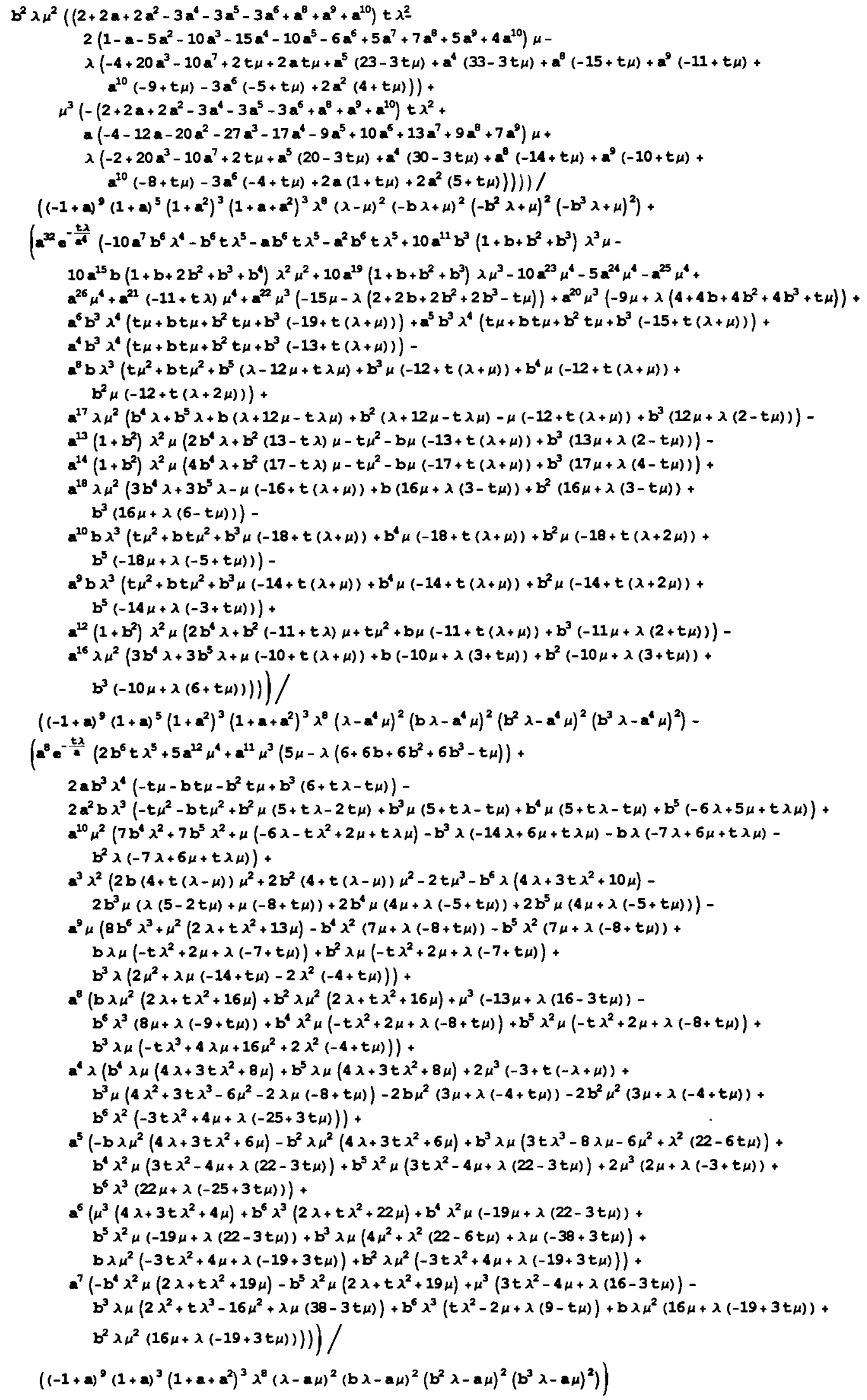




\section{B.2 First 5 Terms for $\operatorname{Gamma}(2, \lambda)$ Operating \& $\operatorname{Gamma}(2, \mu)$ Repair}

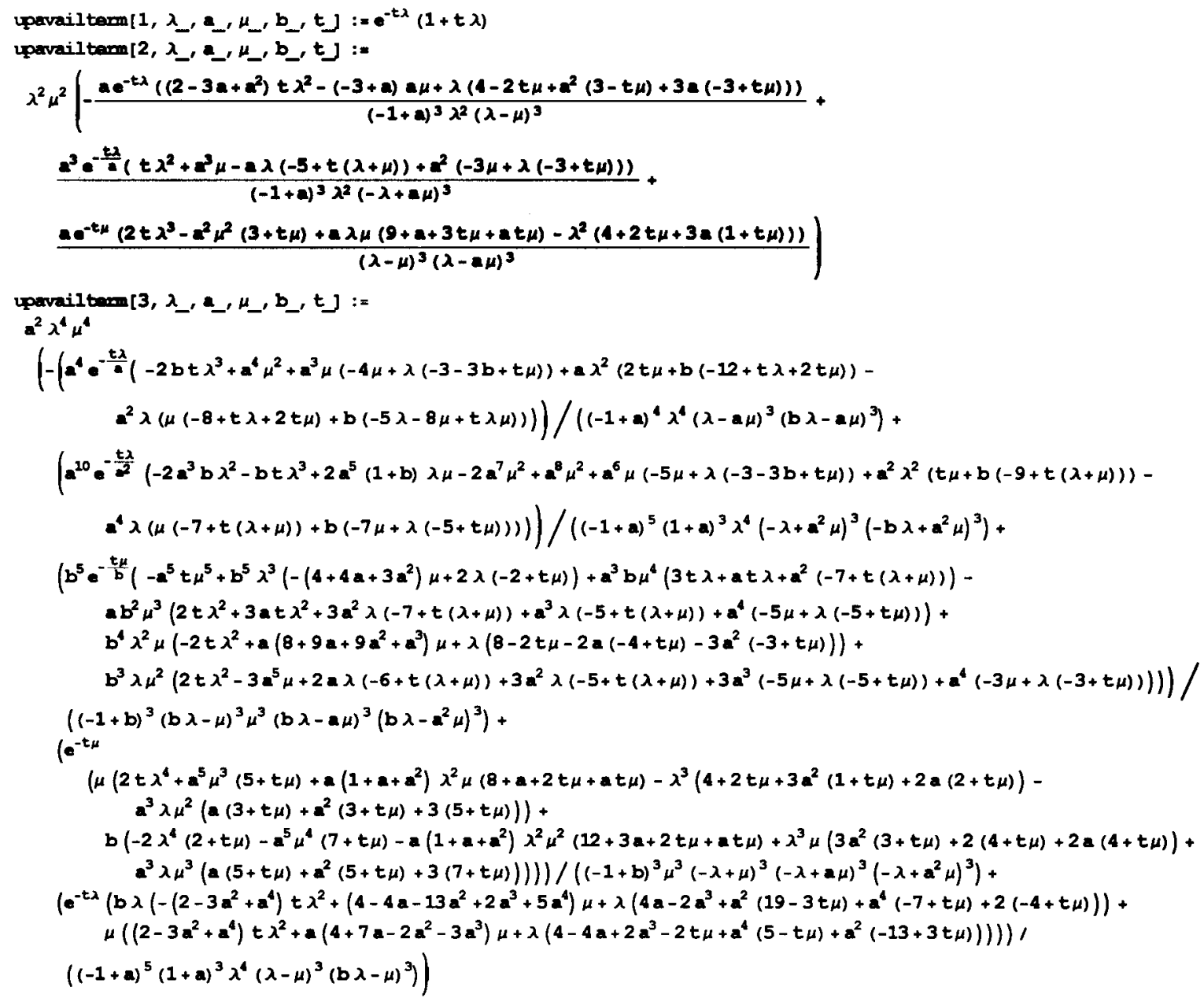




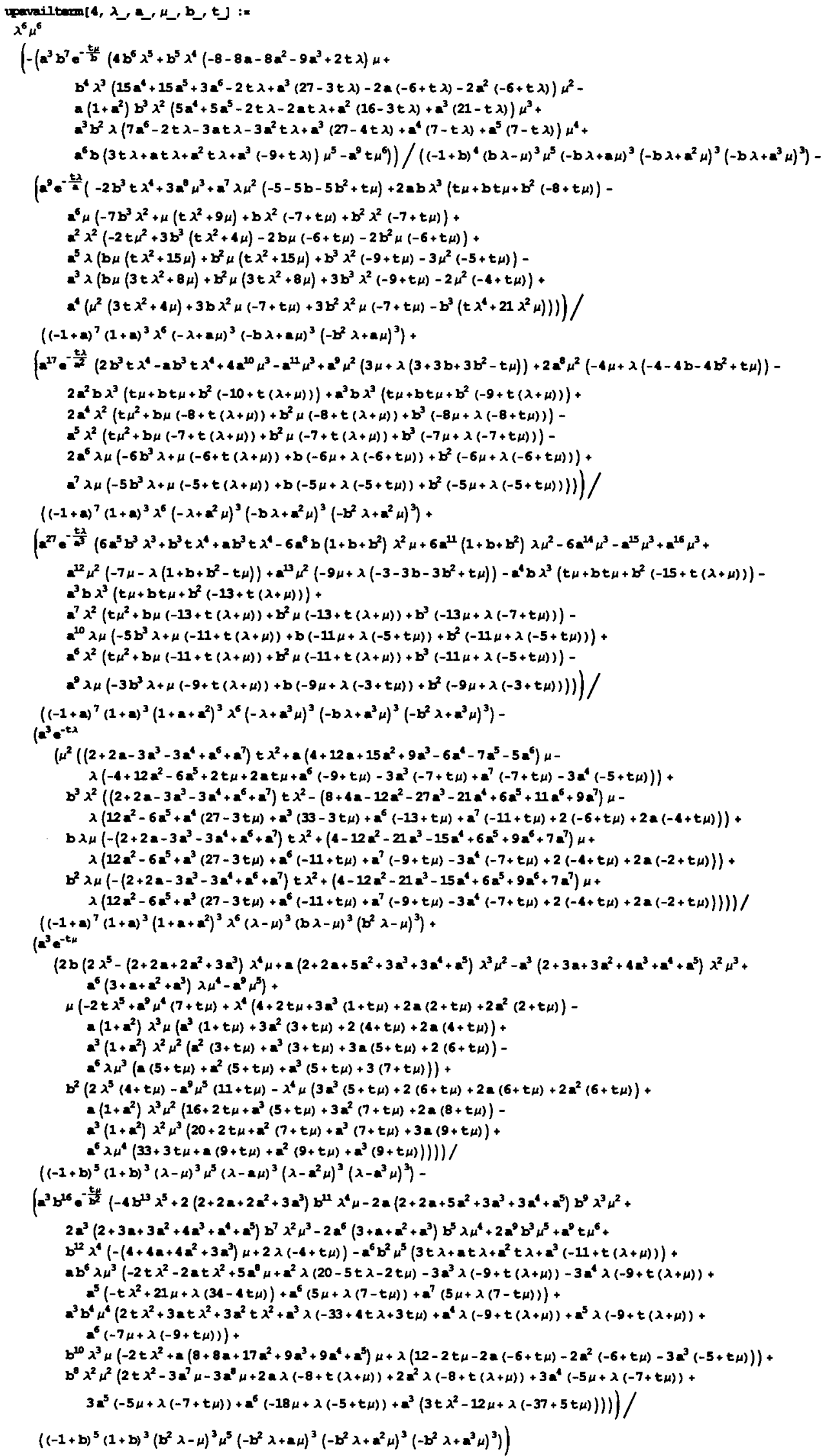


upavailterm [5, $\left.\lambda_{-}, a_{-}, \mu_{-}, b_{-}, t\right]:=$

$\lambda^{8} \mu^{8}$

$\left(-\left(a^{4} b^{9} e^{-\frac{t \mu}{b}}\left(-8 b^{9} \lambda^{6}+b^{8} \lambda^{5}\left(12+12 a+12 a^{2}+12 a^{3}+15 a^{4}-2 t \lambda\right) \mu-a^{10} b\left(3 t \lambda+a t \lambda+a^{2} t \lambda+a^{3} t \lambda+a^{4}(-11+t \lambda)\right) \mu^{6}+\right.\right.\right.$

$a^{14} t \mu^{7}+b^{7} \lambda^{4}\left(4 \lambda^{2}-\left(16+16 a+32 a^{2}+37 a^{3}+37 a^{4}+21 a^{5}+21 a^{6}+5 a^{7}\right) \mu^{2}+\left(2+2 a+2 a^{2}+2 a^{3}+3 a^{4}\right) t \lambda \mu^{2}\right)+$

$b^{6} \lambda^{3} \mu\left(-\left(8+8 a+8 a^{2}+8 a^{3}+9 a^{4}\right) \lambda^{2}+2 t \lambda^{3}+a^{3}\left(20+20 a+47 a^{2}+47 a^{3}+54 a^{4}+34 a^{5}+34 a^{6}+7 a^{7}+7 a^{8}\right) \mu^{2}-\right.$ $\left.a\left(2+2 a+4 a^{2}+5 a^{3}+5 a^{4}+3 a^{5}+3 a^{6}+a^{7}\right) t \lambda \mu^{2}\right)+$

$a^{6} b^{2} \mu^{5}\left(2 t \lambda^{2}+3 a t \lambda^{2}+3 a^{2} t \lambda^{2}+4 a^{3} t \lambda^{2}+a^{6} \lambda(-9+t \lambda)+a^{7} \lambda(-9+t \lambda)+a^{5} \lambda(-9+2 t \lambda)+\right.$ $\left.a^{4} \lambda(-33+4 t \lambda)-a^{8}\left(9 \lambda+t \mu^{2}\right)\right)+$

$a^{3} b^{3} \mu^{4}\left(-2 t \lambda^{3}-2 a t \lambda^{3}-5 a^{2} t \lambda^{3}+a^{5} \lambda^{2}(27-4 t \lambda)+3 a^{4} \lambda^{2}(9-2 t \lambda)+2 a^{6} \lambda^{2}(17-2 t \lambda)-\right.$ $5 a^{3} \lambda^{2}(-4+t \lambda)+a^{11}(-13+t \lambda) \mu^{2}+a^{9} \lambda\left(7 \lambda+t \mu^{2}\right)+a^{10} \lambda\left(7 \lambda+t \mu^{2}\right)+a^{8} \lambda\left(14 \lambda-t \lambda^{2}+t \mu^{2}\right)+$ $\left.a^{7} \lambda\left(34 \lambda-t \lambda^{2}+3 t \mu^{2}\right)\right)+$

$a b^{4} \lambda \mu^{3}\left(2 t \lambda^{3}+2 a t \lambda^{3}+4 a^{2} \lambda^{2}(-4+t \lambda)+a^{4} \lambda^{2}(-37+5 t \lambda)+a^{3} \lambda^{2}(-16+5 t \lambda)+11 a^{13} \mu^{2}+\right.$ $a^{11}(11-t \lambda) \mu^{2}+a^{12}(11-t \lambda) \mu^{2}+a^{7} \lambda\left(-26 \lambda+t \lambda^{2}-3 t \mu^{2}\right)+a^{5} \lambda\left(-37 \lambda+3 t \lambda^{2}-2 t \mu^{2}\right)+$

$\left.3 a^{6} \lambda\left(-14 \lambda+t \lambda^{2}-t \mu^{2}\right)-2 a^{8} \lambda\left(13 \lambda+2 t \mu^{2}\right)+a^{9}\left(-5 \lambda^{2}+39 \mu^{2}-4 t \lambda \mu^{2}\right)+a^{10}\left(-5 \lambda^{2}+11 \mu^{2}-2 t \lambda \mu^{2}\right)\right)+$

$b^{5} \lambda^{2} \mu^{2}\left(-2 t \lambda^{3}-2 a \lambda^{2}(-6+t \lambda)-2 a^{2} \lambda^{2}(-6+t \lambda)-9 a^{12} \mu^{2}-9 a^{13} \mu^{2}+a^{10}(-42+t \lambda) \mu^{2}+\right.$

$a^{11}(-18+t \lambda) \mu^{2}+2 a^{9}(-21+2 t \lambda) \mu^{2}+2 a^{3} \lambda\left(12 \lambda-t \lambda^{2}+t \mu^{2}\right)+a^{4} \lambda\left(27 \lambda-3 t \lambda^{2}+2 t \mu^{2}\right)+$

$\left.\left.\left.a^{5} \lambda\left(27 \lambda+5 t \mu^{2}\right)+3 a^{7}\left(5 \lambda^{2}-11 \mu^{2}+2 t \lambda \mu^{2}\right)+a^{8}\left(3 \lambda^{2}-33 \mu^{2}+4 t \lambda \mu^{2}\right)+a^{6}\left(15 \lambda^{2}-24 \mu^{2}+5 t \lambda \mu^{2}\right)\right)\right)\right) /$

$\left((-1+b)^{7}(1+b)^{3}(b \lambda-\mu)^{3} \mu^{7}(b \lambda-a \mu)^{3}\left(b \lambda-a^{2} \mu\right)^{3}\left(b \lambda-a^{3} \mu\right)^{3}\left(b \lambda-a^{4} \mu\right)^{3}\right)-$

$\left(a^{4} b^{33} e^{-\frac{t \mu}{b^{3}}}\left(12 b^{23} \lambda^{6}+4 b^{24} \lambda^{6}-6\left(2+2 a+2 a^{2}+2 a^{3}+3 a^{4}\right) b^{20} \lambda^{5} \mu+6 a\left(2+2 a+4 a^{2}+5 a^{3}+5 a^{4}+3 a^{5}+3 a^{6}+a^{7}\right) b^{17} \lambda^{4} \mu^{2}-\right.\right.$

$6 a^{3}\left(2+2 a+5 a^{2}+5 a^{3}+6 a^{4}+4 a^{5}+4 a^{6}+a^{7}+a^{8}\right) b^{14} \lambda^{3} \mu^{3}+6 a^{6}\left(2+3 a+3 a^{2}+4 a^{3}+4 a^{4}+2 a^{5}+a^{6}+a^{7}\right) b^{11} \lambda^{2} \mu^{4}-$

$6 a^{10}\left(3+a+a^{2}+a^{3}+a^{4}\right) b^{8} \lambda \mu^{5}+6 a^{14} b^{5} \mu^{6}+a^{14} t \mu^{7}+a^{14} b t \mu^{7}+b^{22} \lambda^{5}\left(\left(4+4 a+4 a^{2}+4 a^{3}+3 a^{4}\right) \mu+\lambda(16-2 t \mu)\right)+$

$b^{21} \lambda^{5}\left(-3 a^{4} \mu-2 \lambda(-6+t \mu)\right)-a^{10} b^{4} \mu^{6}\left(3 t \lambda+a t \lambda+a^{2} t \lambda+a^{3} t \lambda+a^{4}(-17+t(\lambda+\mu))\right)-$

$a^{10} b^{3} \mu^{6}\left(3 t \lambda+a t \lambda+a^{2} t \lambda+a^{3} t \lambda+a^{4}(-15+t(\lambda+\mu))\right)-$

$a\left(1+a+a^{2}+a^{3}+a^{4}\right) b^{12} \lambda^{2} \mu^{3}\left(-2 t \lambda^{2}-a^{3} t \lambda^{2}+12 a^{5} \mu+3 a^{8} \mu-3 a^{4} \lambda(-11+t \mu)-2 a^{2} \lambda(-12+t(\lambda+\mu))+\right.$

$\left.a^{6}(3 \mu+\lambda(9-t \mu))\right)-a\left(1+a+a^{2}+a^{3}+a^{4}\right) b^{13} \lambda^{2} \mu^{3}$

$\left(-2 t \lambda^{2}-a^{3} t \lambda^{2}+16 a^{5} \mu+5 a^{8} \mu-3 a^{4} \lambda(-13+t \mu)-2 a^{2} \lambda(-14+t(\lambda+\mu))+a^{6}(5 \mu+\lambda(11-t \mu))\right)+$

$a^{3} b^{9} \lambda \mu^{4}\left(-2 t \lambda^{2}-2 a t \lambda^{2}-5 a^{2} t \lambda^{2}+5 a^{11} \mu+a^{5} \lambda(39-4 t \lambda-3 t \mu)+a^{3} \lambda(28-5 t \lambda-2 t \mu)-\right.$

$2 a^{6} \lambda(-25+2 t(\lambda+\mu))-3 a^{4} \lambda(-13+t(2 \lambda+\mu))+a^{7}\left(-t \lambda^{2}+21 \mu+\lambda(50-4 t \mu)\right)+$

$\left.a^{8}\left(-t \lambda^{2}+5 \mu+\lambda(22-2 t \mu)\right)+a^{9}(5 \mu+\lambda(11-t \mu))+a^{10}(5 \mu+\lambda(11-t \mu))\right)+$

$a^{3} b^{10} \lambda \mu^{4}\left(-2 t \lambda^{2}-2 a t \lambda^{2}-5 a^{2} t \lambda^{2}+7 a^{11} \mu+a^{5} \lambda(45-4 t \lambda-3 t \mu)+a^{3} \lambda(32-5 t \lambda-2 t \mu)-\right.$

$2 a^{6} \lambda(-29+2 t(\lambda+\mu))-3 a^{4} \lambda(-15+t(2 \lambda+\mu))+a^{7}\left(-t \lambda^{2}+27 \mu+\lambda(58-4 t \mu)\right)+$

$\left.a^{8}\left(-t \lambda^{2}+7 \mu+\lambda(26-2 t \mu)\right)+a^{9}(7 \mu+\lambda(13-t \mu))+a^{10}(7 \mu+\lambda(13-t \mu))\right)+$

$a^{6} b^{7} \mu^{5}\left(2 t \lambda^{2}+3 a t \lambda^{2}+3 a^{2} t \lambda^{2}+4 a^{3} t \lambda^{2}+a^{4} \lambda(-51+4 t \lambda+3 t \mu)+a^{6} \lambda(-15+t(\lambda+\mu))+\right.$

$\left.a^{7} \lambda(-15+t(\lambda+\mu))+a^{5} \lambda(-15+t(2 \lambda+\mu))+a^{8}(-9 \mu+\lambda(-15+t \mu))\right)+$

$a^{6} b^{6} \mu^{5}\left(2 t \lambda^{2}+3 a t \lambda^{2}+3 a^{2} t \lambda^{2}+4 a^{3} t \lambda^{2}+a^{4} \lambda(-45+4 t \lambda+3 t \mu)+a^{6} \lambda(-13+t(\lambda+\mu))+\right.$

$\left.a^{7} \lambda(-13+t(\lambda+\mu))+a^{5} \lambda(-13+t(2 \lambda+\mu))+a^{8}(-7 \mu+\lambda(-13+t \mu))\right)+$

$b^{16} \lambda^{3} \mu^{2}\left(-2 t \lambda^{2}+18 a^{9} \mu+3 a^{10} \mu+3 a^{11} \mu-2 a \lambda(-12+t(\lambda+\mu))-2 a^{2} \lambda(-12+t(\lambda+\mu))+\right.$ $a^{4}\left(-3 t \lambda^{2}+12 \mu+\lambda(57-5 t \mu)\right)+a^{5}(27 \mu+\lambda(57-5 t \mu))+a^{6}(27 \mu+\lambda(33-3 t \mu))+$

$\left.a^{7}(30 \mu+\lambda(33-3 t \mu))+a^{8}(18 \mu+\lambda(9-t \mu))-2 a^{3}\left(t \lambda^{2}-6 \mu+2 \lambda(-12+t \mu)\right)\right)+$

$b^{15} \lambda^{3} \mu^{2}\left(-2 t \lambda^{2}+10 a^{9} \mu+a^{10} \mu+a^{11} \mu-2 a \lambda(-10+t(\lambda+\mu))-2 a^{2} \lambda(-10+t(\lambda+\mu))+\right.$

$a^{4}\left(-3 t \lambda^{2}+8 \mu+\lambda(47-5 t \mu)\right)+a^{5}(17 \mu+\lambda(47-5 t \mu))+a^{6}(17 \mu+\lambda(27-3 t \mu))+$

$\left.a^{7}(18 \mu+\lambda(27-3 t \mu))+a^{8}(10 \mu+\lambda(7-t \mu))-2 a^{3}\left(t \lambda^{2}-4 \mu+2 \lambda(-10+t \mu)\right)\right)+$

$b^{19} \lambda^{4} \mu\left(2 t \lambda^{2}-a\left(8+8 a+16 a^{2}+17 a^{3}+17 a^{4}+9 a^{5}+9 a^{6}+a^{7}\right) \mu+\right.$

$\left.\lambda\left(-20+2 t \mu+2 a(-10+t \mu)+2 a^{2}(-10+t \mu)+2 a^{3}(-10+t \mu)+3 a^{4}(-9+t \mu)\right)\right)+$

$b^{18} \lambda^{4} \mu\left(2 t \lambda^{2}+a\left(-4-4 a-8 a^{2}-7 a^{3}-7 a^{4}-3 a^{5}-3 a^{6}+a^{7}\right) \mu+\right.$

$\left.\left.\left.\lambda\left(-16+2 t \mu+2 a(-8+t \mu)+2 a^{2}(-8+t \mu)+2 a^{3}(-8+t \mu)+3 a^{4}(-7+t \mu)\right)\right)\right)\right) /$

$\left((-1+b)^{7}(1+b)^{3}\left(1+b+b^{2}\right)^{3}\left(b^{3} \lambda-\mu\right)^{3} \mu^{7}\left(b^{3} \lambda-a \mu\right)^{3}\left(b^{3} \lambda-a^{2} \mu\right)^{3}\left(b^{3} \lambda-a^{3} \mu\right)^{3}\left(b^{3} \lambda-a^{4} \mu\right)^{3}\right)+$ 


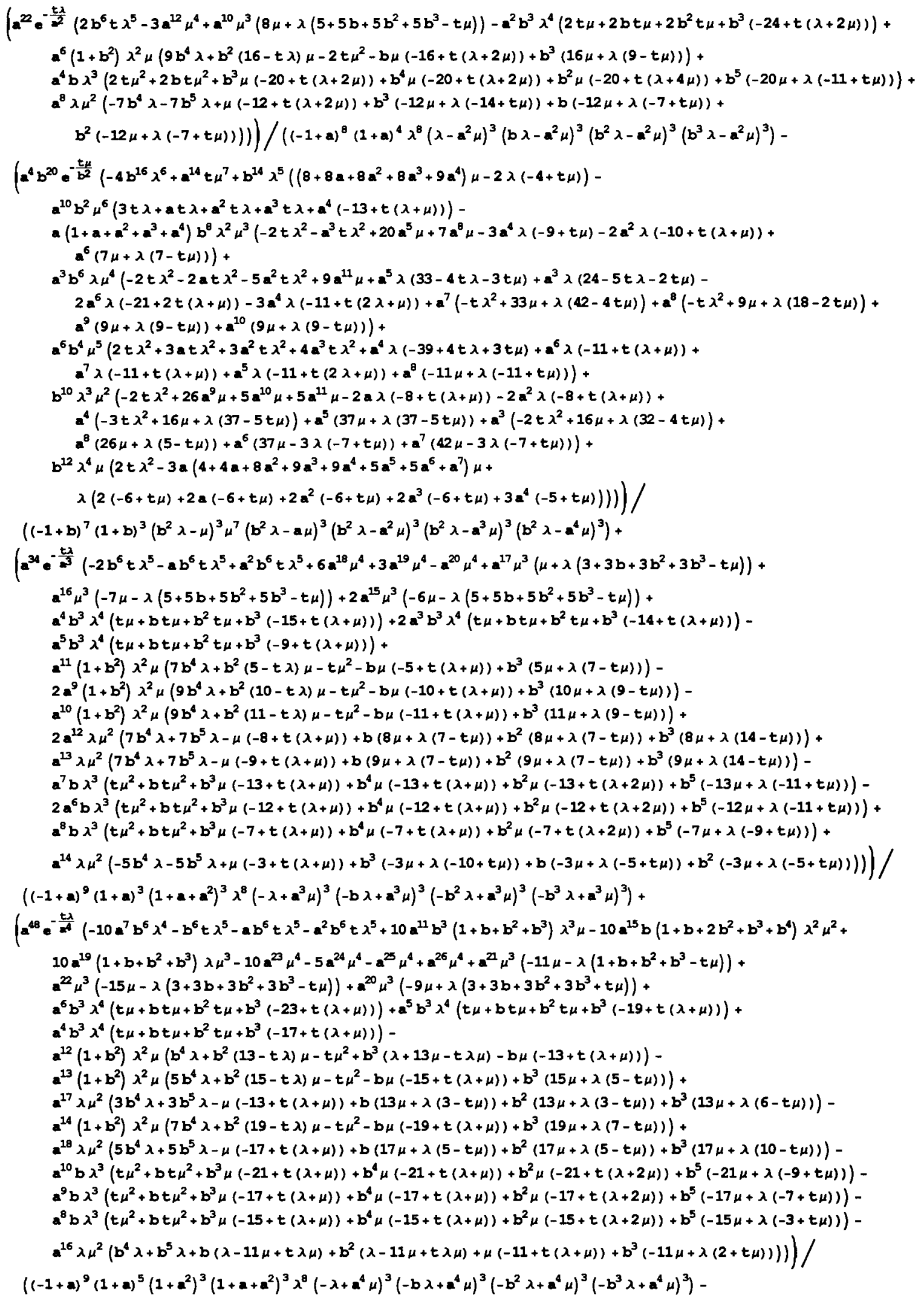




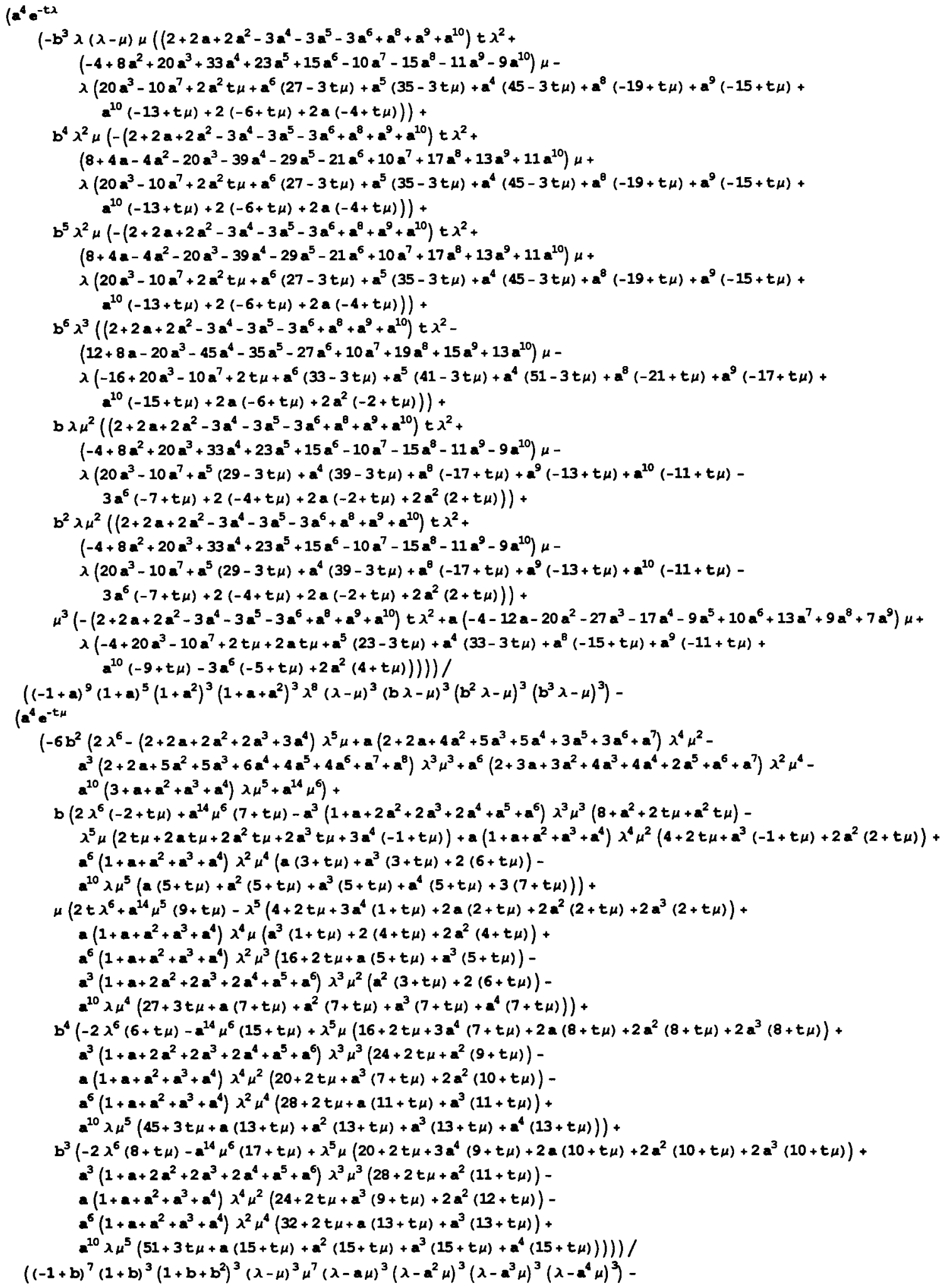




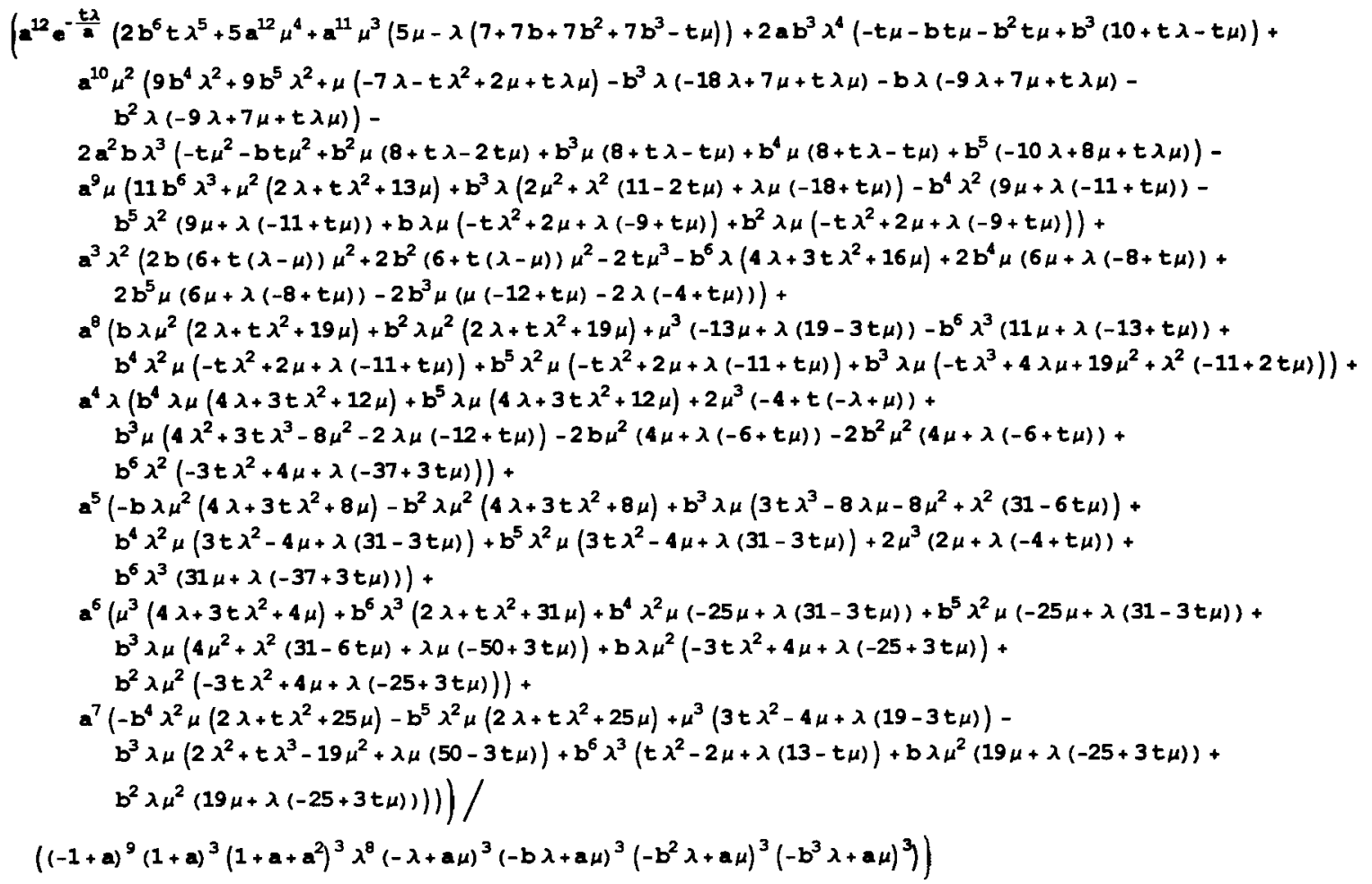




\section{Vita}

\section{Ian Jon Rehmert}

Ian Jon Rehmert was born of Linda Middlestadt and John Rehmert on September 21, 1973 in Baltimore, Maryland. He received his Bachelor of Science Cum Laude in Industrial and Systems Engineering (ISE) on May 13, 1995 from Virginia Polytechnic Institute and State University (Virginia Tech) in Blacksburg, Virginia. While completing his bachelor's degree, Ian was inducted into Alpha Pi Mu and the Golden Key National Honor Society, was a four-year member of the Intercollegiate Men's Lacrosse Team, and was a member and officer of the Delta Upsilon Fraternity. In his final year, Ian worked on a team that earned honorable mention in the International Competition in Modeling. That following summer, he held a position as an operations research intern in the Operations Research department of USAir in Crystal City, Virginia.

On December 18, 1997 Ian earned a Master of Science in ISE and on December 15, 2000 he earned his Doctor of Philosophy in ISE, both with a concentration in Operations Research from Virginia Tech. During his graduate study Ian served as a Graduate Teaching Assistant and Instructor for the ISE department. He assisted with and taught courses in probabilistic operations research, statistical quality control, discrete-event simulation, organizational theory, and industrial cost control. He also served as the Treasurer and President of the student chapter of the Institute for Operations Research and the Management Sciences. Ian was a recipient of both the Pratt Engineering Fellowship and the Cunningham Doctoral Fellowship. For the duration of his graduate study, Ian held positions as a Research Intern and Adjunct Staff Member in the Operational Evaluation Division of the Institute for Defense Analyses (IDA), a think-tank in Alexandria, Virginia that supports the Department of Defense. At IDA he supported projects such as the Close Combat Tactical Trainer, the Bradley Fighting Vehicle, and the Synthetic Environments for National Security Estimates. On October 16, 2000 Ian started as Senior Analyst with skillgames.com, an internet start-up company based in New York, NY. 
Ian's thesis research area is in statistical quality control and his dissertation research area is in availability modeling and analysis. His primary research interests are in the application of probability, statistics, and simulation to solve problems in the service industries. He is a member of the Institute for Operations Research and the Management Sciences. 